\title{
Compressible Navier-Stokes system with general inflow-outflow boundary data
}

\author{
T. Chang * \\ B.J. Jin ${ }^{\dagger}$ \\ A. Novotný $\ddagger$
}

April 2, 2018

Center for Mathematical Analysis and Computations (CMAC), Building 114, Yonsei University, 50 Yonsei-ro Seodaemun-gu, Seoul, 03722, Republic of Korea

and

Department of Mathematics Education, Mokpo National University, Muan 534-729, South Korea

and

IMATH, EA 2134, Université du Sud Toulon-Var

BP 20132, 83957 La Garde, France

\begin{abstract}
We prove existence of weak solutions to the compressible Navier-Stokes equations in barotropic regime (adiabatic coefficient $\gamma>3 / 2$, in three dimensions, $\gamma>1$ in two dimensions) with large velocity prescribed at the boundary and large density prescribed at the inflow boundary of a bounded sufficiently smooth domain, without any restriction neither on the shape of the inflow/outflow boundaries nor on the shape of the domain. The result applies also to pressure laws that are non monotone on a compact portion of interval $[0, \infty)$.
\end{abstract}

Keywords: Compressible Navier-Stokes system, inhomogeneous boundary conditions, weak solutions, renormalized continuity equation, large inflow, large outflow

\footnotetext{
*The work of T.Ch. has been supported by the NRF grant 2015 R1A5A1009350.
}

$\dagger$ The work of B.J.J. has been supported by NRF grant 2016R1D1A1B03934133

${ }^{\ddagger}$ The work of A.N. has been supported by the NRF grant 2015 R1A5A1009350. 


\section{Introduction}

We consider the problem of identifying the non steady motion of a compressible viscous fluid driven by general in/out flux boundary conditions on general bounded domains. Specifically, the mass density $\varrho=\varrho(t, x)$ and the velocity $\mathbf{u}=\mathbf{u}(t, x),(t, x) \in I \times \Omega \equiv Q_{T}, I=(0, T)$ of the fluid satisfy the Navier-Stokes system,

$$
\begin{aligned}
\partial_{t} \varrho+\operatorname{div}_{x}(\varrho \mathbf{u}) & =0 \\
\partial_{t}(\varrho \mathbf{u})+\operatorname{div}_{x}(\varrho \mathbf{u} \otimes \mathbf{u})+\nabla_{x} p(\varrho) & =\operatorname{div}_{x} \mathbb{S}\left(\nabla_{x} \mathbf{u}\right), \\
\mathbb{S}\left(\nabla_{x} \mathbf{u}\right) & =\mu\left(\nabla_{x} \mathbf{u}+\nabla_{x}^{t} \mathbf{u}\right)+\lambda \operatorname{div}_{x} \mathbf{u} \mathbb{I}, \quad \mu>0, \quad \lambda \geq 0
\end{aligned}
$$

in $\Omega \subset R^{d}, d=2,3$, where $p=p(\varrho)$ is the barotropic pressure. The system is endowed with initial conditions

$$
\varrho(0)=\varrho_{0}, \quad \varrho \mathbf{u}(0)=\varrho_{0} \mathbf{u}_{0}
$$

We consider general boundary conditions,

$$
\left.\mathbf{u}\right|_{\partial \Omega}=\mathbf{u}_{B},\left.\varrho\right|_{\Gamma_{\text {in }}}=\varrho_{B},
$$

where

$$
\Gamma_{\text {in }}=\left\{x \in \partial \Omega \mid \mathbf{u}_{B} \cdot \mathbf{n}<0\right\}, \Gamma_{\text {out }}=\left\{x \in \partial \Omega \mid \mathbf{u}_{B} \cdot \mathbf{n}>0\right\} .
$$

We concentrate on the inflow/outflow phenomena, we have therefore deliberately omitted the contribution of external forces $\varrho \mathbf{f}$. Nevertheless, all results of this paper remain valid also in the presence of external forces.

Investigation and better insight to the equations in this setting is important for many real world applications. In fact this is a natural and basic abstract setting for flows in pipelines, wind tunnels, turbines to name a few concrete examples. In spite of this fact the problem in its full generality resists to all attempts of its solution for decades. To the best of our knowledge, this is the first work ever treating this system for large boundary data in a very large class of bounded domains.

Indeed, the only available results on the existence of strong solutions in setting (1.1-1.6) are on a short time interval or deal with small boundary data perturbations of an equilibrium state, see e.g. Valli, Zajaczkowski [23].

The only results on the existence of weak solutions for large flows for system (1.1-1.6) with large boundary data are available in papers by Novo [16] (where the domain is a ball and the incoming/outgoing velocity field is constant) or by Girinon [13], where the domain is more general but the inflow boundary has to be convex set included in a cone, and the velocity at the inflow boundary has to satisfy so called no reflux condition.

In the steady case, the problem with large boundary conditions is open for barotropic flows. It was solved only recently for the constitutive law of pressure of so called hard sphere model (when $\lim _{\varrho \rightarrow \varrho} p(\varrho)=\infty$ for some $\bar{\varrho}>0$ ), see [10]. There are several results dealing with data close to equilibrium flows, see Plotnikov, Ruban, Sokolowski [20], [21], Mucha, Piasecki [15], Piasecki [18], Piasecki and Pokorny [19] among others. 
Our goal is to establish the existence of a weak solution $(\varrho, \mathbf{u})$ to problem (1.1-1.6) for general large boundary data $\varrho_{B}, \mathbf{u}_{B}$ in an arbitrary bounded sufficiently smooth domain with no geometric restrictions on the inflow boundary. Such general result requires a completely different approach to the construction of solutions than the approach employed by Novo or Girinon. We suggest a new (spatially local) method of construction of solutions via regularization of the continuity equation by a specific non homogenous parabolic boundary value problem, instead of using transport equation based approximation as in [16] or [13]. This approach allows to remove the restrictions imposed on the domain and data.

Another novelty with respect to the two above mentioned papers is the fact that we include in our investigation the pressure laws that may be non monotone on a compact portion of interval $[0, \infty)$, in the spirit of [8]. (It is to be noticed that a method allowing non monotone pressure laws on a non compact portion of $[0, \infty)$ was recently suggested in [1], but this method does not work if growth of $p$ (expressed through coefficient $\gamma$ ) is less than $9 / 5$ and it is not clear whether it would work with the non homogenous boundary conditions.)

The paper is organized as follows. In Section 2 we define weak solutions to the problem and state the main theorem (Theorem 2.4). In Section 4 the approximated problem (including two small parameters $\varepsilon>0$ and $\delta>0)$ is specified and its solvability is proved. Limit $\varepsilon \rightarrow 0$ is performed in Section 5 and limit $\delta \rightarrow 0$ in Section 6 . At each stage of the convergence proof from the approximate system to the original system $(\varepsilon \rightarrow 0$ and $\delta \rightarrow 0$, respectively) our approach follows closely the Lions approach [14] (for $\varepsilon \rightarrow 0$ ) and Feireisl's approach [11] (for $\delta \rightarrow 0$ ). This includes the main tools as effective viscous flux identity, oscillations defect measure and renormalization techniques for the continuity equation. The first two tools are local, and remain essentially unchanged (with respect to their use in the case of homogenous Dirichlet boundary conditions), while the third tool - the renormalization technique for the continuity equation introduced in Di Perna-Lions [6] (in the case of squared integrable densities) and in Feireisl [11] (in the case of non squared integrable densities) - has to be essentially modified in order to be able to accommodate general non homogenous boundary data. This topic is investigated in Section 3 (and applied in Sections 5.4 (for the limit $\varepsilon \rightarrow 0$ )) and 6.5 (for the limit $\delta \rightarrow 0$ )). Besides the original approximation presented in Section 4 (that allows to treat the outflow/inflow problem in full generality, without geometric restrictions on the form and position of the inflow/outflow boundaries, in contrast with all previous treatments of this problem) the content of Sections 3, 5.4, 6.5 represents the main novelty of this paper. The results on the renormalized continuity equation formulated in Lemmas 3.1, 3.2 are of independent interest within the context of the theory of compressible fluids.

\section{Main result}

In order to avoid additional technicalities, we suppose that the boundary data satisfy

$$
\mathbf{u}_{B} \in C^{2}\left(\partial \Omega ; R^{d}\right), \varrho_{B} \in C(\partial \Omega) .
$$

In agreement with the standard existence theory in the absence of inflow/outflow, we assume for pressure

$$
p=p-\mathfrak{p}, p \in C[0, \infty) \cap C^{1}(0, \infty), p(0)=0,
$$




$$
\begin{gathered}
\forall \varrho>0, p^{\prime}(\varrho)>\max \left\{0, a_{1} \varrho^{\gamma-1}-b\right\}, p(\varrho) \leq a_{2} \varrho^{\gamma}+b, \\
\mathfrak{p} \in C_{c}^{2}[0, \infty), \mathfrak{p} \geq 0, \mathfrak{p}^{\prime}(0)=0,
\end{gathered}
$$

where $\gamma>1$ and $a_{1}, a_{2}, b>0$. We allow, in general, a non-monotone pressure $p$. If $\mathfrak{p}=0$ then the pressure is monotone $p=p$ and conditions (2.2) includes the isentropic pressure law $p(\varrho)=a \varrho^{\gamma}, a>0$, $\gamma>1$ which can be taken as a particular case. In general the splitting (2.2) to strictly increasing and bounded negative compactly supported functions complies with pressure laws that obey assumptions

$$
a_{1} \varrho^{\gamma-1}-b \leq p^{\prime}(\varrho), \quad p(\varrho)<b+a_{2} \varrho^{\gamma}, p(0)=0,
$$

where $p(\varrho) \geq 0$ in a (small) right neighborhood of 0 . We notice that the very latter condition (or $\mathfrak{p}^{\prime}(0)=0$ ) is not needed in the homogenous case, cf. Feireisl [8], [7]; it is specific for the non-homogenous problem. It enters into the game only in order to treat signs of the boundary terms arising from the non-zero boundary conditions in the a priory estimates (see Section 4.3.3 for more details).

For further convenience, it will be useful to introduce Helmholtz functions:

$$
H(\varrho)=\varrho \int_{0}^{\varrho} \frac{p(z)}{z^{2}} \mathrm{~d} z, \quad \mathscr{H}(\varrho)=\varrho \int_{0}^{\varrho} \frac{p(z)}{z^{2}} \mathrm{~d} z, \quad \mathfrak{H}(\varrho)=-\varrho \int_{0}^{\varrho} \frac{\mathfrak{p}(z)}{z^{2}} \mathrm{~d} z
$$

and relative energy functions

$$
\begin{gathered}
E(\varrho \mid r)=H(\varrho)-H^{\prime}(r)(\varrho-r)-H(r), \quad \mathscr{E}(\varrho \mid r)=\mathscr{H}(\varrho)-\mathscr{H}^{\prime}(r)(\varrho-r)-\mathscr{H}(r), \\
\mathfrak{E}(\varrho \mid r)=\mathfrak{H}(\varrho)-\mathfrak{H}^{\prime}(r)(\varrho-r)-\mathfrak{H}(r) .
\end{gathered}
$$

We begin with the definition of weak solutions to system (1.1-1.6).

Definition 2.1 [Weak solutions to system (1.1-1.6)]

We say that $(\varrho, \mathbf{u})$ is a bounded energy weak solution of problem (1.1-1.6) if:

1. It belongs to functional spaces:

$$
\varrho \in L^{\infty}\left(0, T ; L^{\gamma}(\Omega)\right), 0 \leq \varrho \text { a.a. in }(0, T) \times \Omega, \mathbf{u} \in L^{2}\left(0, T ; W^{1,2}\left(\Omega ; R^{d}\right)\right),\left.\mathbf{u}\right|_{I \times \partial \Omega}=\mathbf{u}_{B}
$$

2. Function $\varrho \in C_{\text {weak }}\left([0, T], L^{\gamma}(\Omega)\right)^{1}$ and the integral identity

$$
\int_{\Omega} \varrho(\tau, \cdot) \varphi(\tau, \cdot) \mathrm{d} x-\int_{\Omega} \varrho_{0}(\cdot) \varphi(0, \cdot) \mathrm{d} x=\int_{0}^{\tau} \int_{\Omega}\left(\varrho \partial_{t} \varphi+\varrho \mathbf{u} \cdot \nabla_{x} \varphi\right) \mathrm{d} x \mathrm{~d} t-\int_{0}^{\tau} \int_{\Gamma_{\mathrm{in}}} \varrho_{B} \mathbf{u}_{B} \cdot \mathbf{n} \varphi \mathrm{d} S_{x} \mathrm{~d} t
$$

holds for any $\tau \in[0, T]$ and $\varphi \in C_{c}^{1}\left([0, T] \times\left(\Omega \cup \Gamma_{\text {in }}\right)\right)$.

\footnotetext{
${ }^{1}$ We say that $f \in C_{\text {weak }}\left([0, T], L^{p}(\Omega)\right)$ iff $\int_{\Omega} f \varphi \mathrm{d} x \in C[0, T]$ for all $\varphi \in L^{p^{\prime}}(\Omega)$
} 
3. Function $\varrho \mathbf{u} \in C_{\text {weak }}\left([0, T], L^{\frac{2 \gamma}{\gamma+1}}\left(\Omega ; R^{d}\right)\right)$, and the integral identity

$$
\begin{gathered}
\int_{\Omega} \varrho \mathbf{u}(\tau, \cdot) \cdot \boldsymbol{\varphi}(\tau, \cdot) \mathrm{d} x-\int_{\Omega} \varrho_{0} \mathbf{u}_{0}(\cdot) \boldsymbol{\varphi}(0, \cdot) \mathrm{d} x \\
=\int_{0}^{\tau} \int_{\Omega}\left(\varrho \mathbf{u} \cdot \partial_{t} \varphi+\varrho \mathbf{u} \otimes \mathbf{u}: \nabla_{x} \boldsymbol{\varphi}+p(\varrho) \operatorname{div}_{x} \boldsymbol{\varphi}-\mathbb{S}\left(\nabla_{x} \mathbf{u}\right): \nabla_{x} \boldsymbol{\varphi}\right) \mathrm{d} x \mathrm{~d} t
\end{gathered}
$$

holds for any $\tau \in[0, T]$ and any $\varphi \in C_{c}^{1}\left([0, T] \times \Omega ; R^{d}\right)$.

4. There exists a Lipschitz extension $\mathbf{u}_{\infty} \in W^{1, \infty}\left(\Omega ; R^{d}\right)$ of $\mathbf{u}_{B}$ whose divergence is non negative in a certain interior neighborhood of $\partial \Omega$, i.e.

$$
\operatorname{div} \mathbf{u}_{\infty} \geq 0 \text { a.e. in } \hat{U}_{h}^{-} \equiv\{x \in \Omega \mid \operatorname{dist}(x, \partial \Omega)<h\}, h>0
$$

such that the energy inequality

$$
\begin{array}{r}
\int_{\Omega}\left(\frac{1}{2} \varrho\left|\mathbf{u}-\mathbf{u}_{\infty}\right|^{2}+H(\varrho)\right)(\tau) \mathrm{d} x+\int_{0}^{\tau} \int_{\Omega} \mathbb{S}\left(\nabla_{x}\left(\mathbf{u}-\mathbf{u}_{\infty}\right)\right): \nabla_{x}\left(\mathbf{u}-\mathbf{u}_{\infty}\right) \mathrm{d} x \mathrm{~d} t \\
\leq \int_{\Omega}\left(\frac{1}{2} \varrho_{0}\left|\mathbf{u}_{0}-\mathbf{u}_{\infty}\right|^{2}+H\left(\varrho_{0}\right)\right) \mathrm{d} x-\int_{0}^{\tau} \int_{\Omega} p(\varrho) \operatorname{div} \mathbf{u}_{\infty} \mathrm{d} x \mathrm{~d} t-\int_{0}^{\tau} \int_{\Omega} \varrho \mathbf{u} \cdot \nabla_{x} \mathbf{u}_{\infty} \cdot\left(\mathbf{u}-\mathbf{u}_{\infty}\right) \mathrm{d} x \mathrm{~d} t \\
-\int_{0}^{\tau} \int_{\Omega} \mathbb{S}\left(\nabla_{x} \mathbf{u}_{\infty}\right): \nabla_{x}\left(\mathbf{u}-\mathbf{u}_{\infty}\right) \mathrm{d} x \mathrm{~d} t-\int_{0}^{\tau} \int_{\Gamma_{\text {in }}} H\left(\varrho_{B}\right) \mathbf{u}_{B} \cdot \mathbf{n} \mathrm{d} S_{x} \mathrm{~d} t-\underline{H} \int_{0}^{\tau} \int_{\Gamma_{\text {out }}} \mathbf{u}_{B} \cdot \mathbf{n} \mathrm{d} S_{x} \mathrm{~d} t
\end{array}
$$

holds. In inequality (2.9),

$$
\underline{H}=\inf _{\varrho>0} H(\varrho)>-\infty .
$$

Remark 2.1. 1. An extension $\mathbf{u}_{\infty}$ of $\mathbf{u}_{B}$ verifying (2.8) always exists, due to the following lemma (see [13, Lemma 3.3]).

Lemma 2.2. Let $\mathbf{V} \in W^{1, \infty}\left(\partial \Omega ; R^{d}\right)$ be a Lipschitz vector field on the boundary $\partial \Omega$ of a bounded Lipschitz domain $\Omega$. Then there is $h>0$ and a vector field

$$
\mathbf{V}_{\infty} \in W^{1, \infty}\left(R^{3}\right) \cap C_{c}\left(R^{d}\right), \operatorname{div} \mathbf{V}_{\infty} \geq 0 \text { a.e. in } \hat{U}_{h}
$$

verifying $\left.\mathbf{V}_{\infty}\right|_{\partial \Omega}=\mathbf{V}$, where

$$
\hat{U}_{h}=\left\{x \in R^{3} \mid \operatorname{dist}(x, \partial \Omega)<h\right\} .
$$

2. A brief inspection of formula (2.10) gives the estimate of value $\underline{H}$,

$$
\underline{H} \geq-\sup _{\varrho \in(0,1)} p(\varrho)-\bar{\varrho} \sup _{\varrho>1} \mathfrak{p}(\varrho)>-\infty
$$

provided suppp $\subset[0, \bar{r}]$, where $\bar{r}>1$ without loss of generality. 
3. Equation (2.6) implies the total mass inequality

$$
\int_{\Omega} \varrho(\tau) \mathrm{d} x \leq \int_{\Omega} \varrho_{0} \mathrm{~d} x-\int_{0}^{\tau} \int_{\Gamma_{\mathrm{in}}} \varrho_{B} \mathbf{u}_{B} \cdot \mathbf{n} \mathrm{d} S_{x} \mathrm{~d} t
$$

for all $\tau \in[0, T]$. To see it, it is enough to take for test functions a convenient sequence $\varphi=\varphi_{\delta}$, $\delta>0$ (e.g. the same as suggested in (5.27)) and let $\delta \rightarrow 0$.

Definition 2.2 We say that the couple $(\varrho, \mathbf{u}) \in L^{p}\left(Q_{T}\right) \times L^{2}\left(0, T ; W^{1,2}\left(\Omega, R^{d}\right)\right), p>1$ is a renormalized solution of the continuity equation if $b(\varrho) \in C_{\mathrm{weak}}\left([0, T] ; L^{1}(\Omega)\right)$ and if it satisfies in addition to the continuity equation (2.6) also equation

$$
\begin{gathered}
\int_{\Omega}(b(\varrho) \varphi)(\tau) \mathrm{d} x-\int_{\Omega} b\left(\varrho_{0}\right) \varphi(0) \mathrm{d} x= \\
\int_{0}^{\tau} \int_{\Omega}\left(b(\varrho) \mathbf{u} \cdot \nabla_{x} \varphi-\varphi\left(b^{\prime}(\varrho) \varrho-b(\varrho)\right) \operatorname{div}_{x} \mathbf{u}\right) \mathrm{d} x \mathrm{~d} t-\int_{0}^{\tau} \int_{\Gamma_{\mathrm{in}}} b\left(\varrho_{B}\right) \mathbf{u}_{B} \cdot \mathbf{n} \varphi \mathrm{d} S_{x} \mathrm{~d} t
\end{gathered}
$$

for any $\varphi \in C_{c}^{1}\left([0, T] \times\left(\Omega \cup \Gamma_{\mathrm{in}}\right)\right)$, and any continuously differentiable $b$ with $b^{\prime}$ having a compact support in $[0, \infty)$.

Weak solution to problem (1.1-1.6) satisfying in addition renormalized continuity equation (2.13) is called renormalized weak solution.

Remark 2.3. It can be shown easily by using the Lebesgue dominated convergence theorem that the the family of test functions $b$ in the previous definition can be extended to

$$
b \in C[0, \infty) \cap C^{1}(0, \infty), z b^{\prime}-b \in C[0, \infty),|b(z)| \leq c\left(1+z^{5 p / 6}\right),\left|z b^{\prime}(z)-b(z)\right| \leq c\left(1+z^{p / 2}\right) .
$$

Our main result is the following theorem.

Theorem 2.4. Let $\Omega \subset R^{d}, d=2,3$ be a bounded domain of class $C^{2}$. Let the boundary data $\mathbf{u}_{B}, \varrho_{B}$ satisfy (2.1), where $\min \varrho_{B} \equiv \underline{\varrho}_{B}>0$. Assume that the pressure satisfies hypotheses (2.2) with $\gamma>d / 2$ and the initial data are of finite energy

$$
\int_{\Omega}\left(\frac{1}{2} \varrho_{0} \mathbf{u}_{0}^{2}+H\left(\varrho_{0}\right)\right) \mathrm{d} x<\infty, \quad 0 \leq \varrho_{0}, \int_{\Omega} \varrho_{0} \mathrm{~d} x>0 .
$$

Then for any Lipschitz extension $\mathbf{u}_{\infty}$ of $\mathbf{u}_{B}$ verifying (2.8) problem (1.1-1.6) possesses at least one bounded energy renormalized weak solution $(\varrho, \mathbf{u})$.

Remark 2.5. 1. Theorem 2.4 holds regardless the $(d-1)$-Hausdorff measure of $\Gamma_{\text {in }}$ or $\Gamma_{\text {out }}$ is zero. If the Hausdorff measure $\left|\Gamma_{\text {in }}\right|_{d-1}=0$ then all conditions on $\varrho_{B}$ become irrelevant. The standard theory developed in [11, Theorem 1.1] (see also monographs [14], [7],[17], [9]) covers the case $\left.\mathbf{u}\right|_{(0, T) \times \partial \Omega}=0$ (which is covered by Theorem 2.4 as well) and the case $\left.\mathbf{u} \cdot \mathbf{n}\right|_{(0, T) \times \partial \Omega}=0$ completed with the Navier conditions-eventually with friction- (which is not covered by Theorem 2.4). 
2. Theorem 2.4 still holds provided one considers in the momentum equation at its right hand side term $\varrho \mathbf{f}$ corresponding to large external forces, provided $\mathbf{f} \in L^{\infty}\left(Q_{T}\right)$ (modulo necessary changes in the weak formulation in order to accommodate the presence of this term).

3. Conditions on the regularity $p, \mathfrak{p}, \varrho_{B}$ and $\mathbf{u}_{B}$ in Theorem 2.4 could be slightly weakened, up to $p$ continuous on $[0, \infty)$, locally Lipschitz on $[0, \infty)$ instead of $p \in C[0, \infty) \cap C^{1}(0, \infty), \mathfrak{p}$ Lipschitz differentiable with compact support on $[0, \infty)$ instead of $\mathfrak{p} \in C_{c}^{2}[0, \infty), \varrho_{B} \in L^{\infty}(\partial \Omega)$, $\mathbf{u}_{B} \in W^{1, \infty}(\partial \Omega)$, at expense of some additional technical difficulties.

We shall perform the proof in all details in the case $d=3$ assuming tacitly that both $\Gamma_{\text {in }}$ and $\Gamma_{\text {out }}$ have non zero $(d-1)$-Hausdorff measure. Other cases, namely the case $d=2$ is left to the reader as an exercise.

\section{Renormalized continuity equation with non homogenous data}

\subsection{The case of squared integrable density}

In this section we generalize the Di-Perna, Lions transport theory [6] to the continuity equation with non homogenous boundary data. The main result reads:

Lemma 3.1. Suppose that $\Omega \subset R^{d}, d=2,3$ be a bounded Lipschitz domain and let $\left(\varrho_{B}, \mathbf{u}_{B}\right)$ satisfy assumptions (2.1). Assume that the inflow portion of the boundary $\Gamma_{\text {in }}$ is a $C^{2}$ open $(d-1)$-dimensional manifold. Suppose further that couple $(\varrho, \mathbf{u}) \in L^{2}((0, T) \times \Omega) \times L^{2}\left(0, T ; W^{1,2}\left(\Omega ; R^{3}\right)\right)$ satisfies continuity equation in the weak sense (2.6).

Then $(\varrho, \mathbf{u})$ is also a renormalized solution of the continuity equation (2.6), meaning that it verifies equation (2.13) with any $\varphi \in C_{c}^{1}\left([0, T] \times\left(\Omega \cup \Gamma_{\mathrm{in}}\right)\right)$, and any continuously differentiable $b$ with $b^{\prime}$ having compact support in $[0, \infty)$.

\section{Proof of Lemma 3.1}

We define open set

$$
\mathcal{U}_{h}^{+}\left(\Gamma_{\mathrm{in}}\right) \equiv\left\{\mathbf{x}_{0}+z \mathbf{n}\left(x_{0}\right) \mid 0<z<h, \mathbf{x}_{0} \in \Gamma_{\text {in }}\right\} \cap\left(R^{d} \backslash \bar{\Omega}\right)
$$

and extend the vector field $\mathbf{u}_{B}$ to $\mathscr{U}_{h}^{+}\left(\Gamma_{\text {in }}\right)$,

$$
\tilde{\mathbf{u}}_{B}(x)=\mathbf{u}_{B}\left(\mathbf{x}_{0}\right), \quad x=\mathbf{x}_{0}+z \mathbf{n}\left(\mathbf{x}_{0}\right) \in \mathcal{U}_{h}^{+}\left(\Gamma_{\text {in }}\right) .
$$

If $\Gamma_{\text {in }} \in C^{2}$, such extension always exists and $\tilde{\mathbf{u}}_{B} \in C^{1}\left(\overline{\mathcal{U}_{h}^{+}\left(\Gamma_{\text {in }}\right.}\right)$, cf. Foote [12]. Consider now the flow generated in $\mathcal{U}_{h}^{+}\left(\Gamma_{\text {in }}\right)$ by the field $-\tilde{\mathbf{u}}_{B}$ defined on $\overline{\mathcal{U}_{h}^{+}\left(\Gamma_{\text {in }}\right)}$,

$$
\mathbf{X}^{\prime}\left(s, \mathbf{x}_{0}\right)=-\tilde{\mathbf{u}}_{B}\left(\mathbf{X}\left(s, \mathbf{x}_{0}\right)\right), \mathbf{X}(0)=\mathbf{x}_{0} \in \mathcal{U}_{h}^{+}\left(\Gamma_{\text {in }}\right) \cup \Gamma_{\text {in }} \text {, for } s>0, \mathbf{X}\left(s ; \mathbf{x}_{0}\right) \in \mathcal{U}_{h}^{+}\left(\Gamma_{\text {in }}\right) .
$$


Let

$$
\tilde{\mathcal{U}}_{h}^{+}\left(\Gamma_{\text {in }}\right)=\left\{x \in \mathscr{U}_{h}^{+}\left(\Gamma_{\text {in }}\right) \mid x=\mathbf{X}\left(s, \mathbf{x}_{0}\right) \text { for a certain } \mathbf{x}_{0} \in \Gamma_{\text {in }} \text { and } 0<s<h\right\} .
$$

Employing the local Cauchy-Lipschitz theory for ODEs to equation (3.3) and evoking the differentiability properties of its solutions with respect to the "initial" data (see e.g. the book of Taylor [22, Chapter 1] or of Benzoni-Gavage [2]), we infer that:

1. For any $\mathbf{x}_{0} \in \mathcal{U}_{h}^{+}\left(\Gamma_{\text {in }}\right)$, there is unique $\bar{T}\left(\mathbf{x}_{0}\right)>0$ and $\underline{T}\left(\mathbf{x}_{0}\right)>0$ such that the map $\mathbf{X} \in$ $C^{1}\left(\left(-\underline{T}\left(\mathbf{x}_{0}\right), \bar{T}\left(\mathbf{x}_{0}\right)\right) ; \mathscr{U}_{h}^{+}\left(\Gamma_{\text {in }}\right)\right)$ is a maximal solution of problem (3.3). If $\mathrm{x}_{0} \in \Gamma_{\text {in }}$, then there is unique $\bar{T}\left(\mathbf{x}_{0}\right)>0$ such that the map $\mathbf{X} \in C^{1}\left(\left[0, \bar{T}\left(\mathbf{x}_{0}\right)\right) ; \mathscr{U}_{h}^{+}\left(\Gamma_{\text {in }}\right) \cup \Gamma_{\text {in }}\right)$ is a maximal solution.

2. For any compact $K \subset \mathcal{U}_{h}^{+}\left(\Gamma_{\text {in }}\right) \cup \Gamma_{\text {in }}, \bar{T}_{K} \equiv \inf _{\mathbf{x}_{0} \in K} \bar{T}\left(\mathbf{x}_{0}\right)>0$ and for any compact $K \subset \mathcal{U}_{h}^{+}\left(\Gamma_{\text {in }}\right)$, $\underline{T}_{K} \equiv \inf _{\mathbf{x}_{0} \in K} \underline{T}\left(\mathbf{x}_{0}\right)>0$.

3. For any $\mathbf{z} \in \tilde{\mathscr{U}}_{h}^{+}\left(\Gamma_{\text {in }}\right)$ there is an open ball $B(\mathbf{z})$ centered at $\mathbf{z}$ and $\delta_{\mathbf{z}}>0$ such that $\mathbf{X} \in C^{1}\left(\left[-\delta_{\mathbf{z}}, \delta_{\mathbf{z}}\right] \times\right.$ $\overline{B(\mathbf{z})})$.

In particular, item 1. in the above list implies that the set $\tilde{\mathscr{U}}_{h}^{+}\left(\Gamma_{\text {in }}\right)$ is not empty. With points 2. and 3. at hand, we are ready to show that $\tilde{\mathcal{U}}_{h}^{+}\left(\Gamma_{\text {in }}\right)$ is an open set. Indeed, let $\mathbf{z}_{0}=\mathbf{X}\left(s_{0} ; \mathbf{x}_{0}\right)$, $s_{0} \in(0, \tilde{T}), \tilde{T}=\min \left\{h, \bar{T}\left(\mathbf{x}_{0}\right)\right\}$ with $\mathbf{x}_{0}=\gamma(0)$, where $\gamma: B^{\prime}(0) \rightarrow R^{d}, \gamma(\sigma)=\mathbf{x}_{0}+\mathbb{O}(\sigma, a(\sigma))^{T}$ with $a \in C^{2}\left(B^{\prime}(0) ; R_{+}\right)$representing the local description of $\Gamma_{\text {in }}$ in the vicinity of $\mathbf{x}_{0}$. In the above $\mathbb{O}$ is a fixed orthonormal matrix, $B^{\prime}(0)$ is a $d-1$ dimensional ball centered at 0 , and we may suppose, without loss of generality, that $\nabla_{\sigma} a \neq 0$ in $B^{\prime}(0)$. We may now consider the map

$$
\begin{gathered}
\Phi:\left(-\underline{T}_{K}, \bar{T}_{K}\right) \times B^{\prime}(0) \ni(s, \sigma) \mapsto \mathbf{z} \in \Phi\left(\left(-\underline{T}_{K}, \bar{T}_{K}\right) \times B^{\prime}(0)\right) \subset \tilde{\mathscr{U}}_{h}^{+}\left(\Gamma_{\mathrm{in}}\right), \\
\mathbf{z}=\Phi(s, \sigma)=\mathbf{X}\left(s ; \mathbf{X}\left(s_{0} ; \gamma(\sigma)\right)\right), K=\overline{\left\{\mathbf{X}\left(s_{0}, \gamma(\sigma) \mid \sigma \in B^{\prime}(0)\right\}\right.} .
\end{gathered}
$$

We have clearly $\Phi(0,0)=\mathbf{z}_{0}$. It is a cumbersome calculation to show that

$$
\operatorname{det}\left(\partial_{s} \Phi, \nabla_{\sigma} \Phi\right)(0,0) \neq 0
$$

see [13, Section 3.3.6]. We may therefore apply to this map the implicit function theorem and conclude that there is an open set $(0,0) \in U \subset\left(-\underline{T}_{K}, \bar{T}_{K}\right) \times B^{\prime}(0)$, open set $\mathbf{z}_{0} \in V \subset R^{d}$, and a map $\Psi \in C^{1}(V ; U)$ such that $\Phi \circ \Psi(\mathbf{z})=\mathbf{z}$ for any $\mathbf{z} \in V$. In particular, $V \subset \Phi\left(\left(-\underline{T}_{K}, \bar{T}_{K}\right) \times B^{\prime}(0)\right)$.

We may therefore extend the boundary data $\varrho_{B}$ to $\tilde{\mathcal{U}}_{h}^{+}\left(\Gamma_{\text {in }}\right)$ by setting

$$
\tilde{\varrho}_{B}\left(\mathbf{X}\left(s, \mathbf{x}_{0}\right)\right)=\varrho_{B}\left(\mathbf{x}_{0}\right) \exp \left(\int_{0}^{s} \operatorname{div} \tilde{\mathbf{u}}_{B}\left(\mathbf{X}\left(z, \mathbf{x}_{0}\right)\right) \mathrm{d} z\right) .
$$

Clearly, $\tilde{\varrho}_{B} \in W^{1, \infty}\left(\tilde{\mathcal{U}}_{h}^{+}\left(\Gamma_{\text {in }}\right)\right)$ and

$$
\operatorname{div}_{x}\left(\tilde{\varrho}_{B} \tilde{\mathbf{u}}_{B}\right)=0 \text { in } \tilde{\mathcal{U}}_{h}^{+}\left(\Gamma_{\text {in }}\right),\left.\quad \tilde{\varrho}_{B}\right|_{\Gamma_{\text {in }}}=\varrho_{B} .
$$


Now we put

$$
\tilde{\Omega}_{h}=\Omega \cup \Gamma_{\text {in }} \cup \tilde{\mathscr{U}}_{h}^{+}\left(\Gamma_{\text {in }}\right)
$$

and extend $(\varrho, \mathbf{u})$ from $(0, T) \times \Omega$ to $(0, T) \times \tilde{\Omega}_{h}$ by setting $(\varrho, \mathbf{u})(t, x)=\left(\tilde{\varrho}_{B}, \tilde{\mathbf{u}}_{B}\right)(x),(t, x) \in(0, T) \times$ $\tilde{\mathcal{U}}_{h}^{+}\left(\Gamma_{\text {in }}\right)$. Conserving notation $(\varrho, \mathbf{u})$ for the extended fields, we easily verify that $(\varrho, \mathbf{u}) \in L^{2}((0, T) \times$ $\left.\tilde{\Omega}_{h}\right) \times L^{2}\left(0, T ; W^{1,2}\left(\tilde{\Omega}_{h} ; R^{d}\right)\right)$, and that it satisfies the equation of continuity (1.1), in particular, in the sense of distributions on $(0, T) \times \tilde{\Omega}_{h}$.

Next, we use the regularization procedure due to DiPerna and Lions [6] applying convolution with a family of regularizing kernels obtaining for the regularized function $[\varrho]_{\varepsilon}$,

$$
\partial_{t}[\varrho]_{\varepsilon}+\operatorname{div}_{x}\left([\varrho]_{\varepsilon} \mathbf{u}\right)=R_{\varepsilon} \text { a.e. in }(0, T) \times \tilde{\Omega}_{\varepsilon, h},
$$

where

$$
\tilde{\Omega}_{\varepsilon, h}=\left\{x \in \tilde{\Omega}_{h} \mid \operatorname{dist}\left(x, \partial \tilde{\Omega}_{h}\right)>\varepsilon\right\}, R_{\varepsilon} \equiv \operatorname{div}_{x}\left([\varrho]_{\varepsilon} \mathbf{u}\right)-\operatorname{div}_{x}\left([\varrho \mathbf{u}]_{\varepsilon}\right) \rightarrow 0 \text { in } L_{\mathrm{loc}}^{1}\left((0, T) \times \tilde{\Omega}_{h}\right) \text { as } \varepsilon \rightarrow 0 .
$$

The convergence of $R_{\varepsilon}$ evoked above results from the application of the refined version of the Friedrichs lemma on commutators, see e.g. [6] or [9, Lemma 10.12 and Corollary 10.3].

Multiplying equation $(3.6)$ on $b^{\prime}\left([\varrho]_{\varepsilon}\right)$, we get

$$
\partial_{t} b\left([\varrho]_{\varepsilon}\right)+\operatorname{div}_{x}\left(b\left([\varrho]_{\varepsilon}\right) \mathbf{u}\right)+\left(b^{\prime}\left([\varrho]_{\varepsilon}\right)[\varrho]_{\varepsilon}-b\left([\varrho]_{\varepsilon}\right)\right) \operatorname{div}_{x} \mathbf{u}=b^{\prime}\left([\varrho]_{\varepsilon}\right) R_{\varepsilon}
$$

or equivalently,

$$
\begin{gathered}
\int_{\tilde{\Omega}_{h}} b\left([\varrho]_{\varepsilon}(\tau)\right) \varphi(\tau) \mathrm{d} x-\int_{\tilde{\Omega}_{h}} b\left(\left[\varrho \varrho_{0}\right) \varphi(0) \mathrm{d} x\right. \\
=\int_{0}^{\tau} \int_{\tilde{\Omega}_{h}}\left(b\left([\varrho]_{\varepsilon}\right) \partial_{t} \varphi+b\left([\varrho]_{\varepsilon}\right) \mathbf{u} \cdot \nabla_{x} \varphi-\varphi\left(b^{\prime}\left([\varrho]_{\varepsilon}\right)[\varrho]_{\varepsilon}-b\left([\varrho]_{\varepsilon}\right)\right) \operatorname{div}{ }_{x} \mathbf{u}\right) \mathrm{d} x \mathrm{~d} t-\int_{0}^{\tau} \int_{\tilde{\Omega}_{h}} \varphi b^{\prime}\left([\varrho]_{\varepsilon}\right) R_{\varepsilon} \mathrm{d} x d t
\end{gathered}
$$
for all $\tau \in[0, T]$, for any $\varphi \in C_{c}^{1}\left([0, T] \times \tilde{\Omega}_{h}\right), 0<\varepsilon<\operatorname{dist}\left(\operatorname{supp}(\varphi), \partial \tilde{\Omega}_{h}\right)$. Thus, letting $\varepsilon \rightarrow 0$ we get

$$
\begin{gathered}
\int_{\tilde{\Omega}_{h}} b(\varrho(\tau)) \varphi(\tau) \mathrm{d} x-\int_{\tilde{\Omega}_{h}} b(\varrho) \varphi(0) \mathrm{d} x \\
=\int_{0}^{\tau} \int_{\tilde{\Omega}_{h}}\left(b(\varrho) \partial_{t} \varphi+b(\varrho) \mathbf{u} \cdot \nabla_{x} \varphi-\varphi\left(b^{\prime}(\varrho) \varrho-b(\varrho)\right) \operatorname{div}_{x} \mathbf{u}\right) \mathrm{d} x \mathrm{~d} t
\end{gathered}
$$

for all $\tau \in[0, T]$, for any $\varphi \in C_{c}^{1}\left([0, T] \times \tilde{\Omega}_{h}\right)$. Now we write

$$
\int_{\tilde{\Omega}_{h}} b(\varrho) \mathbf{u} \cdot \nabla_{x} \varphi \mathrm{d} x=\int_{\Omega} b(\varrho) \mathbf{u} \cdot \nabla_{x} \varphi \mathrm{d} x+\int_{\tilde{U}_{h}^{+}\left(\Gamma_{\mathrm{in}}\right)} b(\varrho) \mathbf{u} \cdot \nabla_{x} \varphi \mathrm{dx}
$$

where, due to (3.5), the second integral is equal to

$$
\int_{\Gamma_{\mathrm{in}}} \varphi b\left(\varrho_{B}\right) \mathbf{u}_{B} \cdot \mathbf{n} \mathrm{d} S_{x}+\int_{\tilde{\mathcal{u}}_{h}^{+}\left(\Gamma_{\mathrm{in}}\right)} \varphi\left(\tilde{\varrho}_{B} b^{\prime}\left(\tilde{\varrho}_{B}\right)-b\left(\tilde{\varrho}_{B}\right)\right) \operatorname{div} \tilde{\mathbf{u}}_{B} \mathrm{~d} x
$$


We notice that $\varphi$ is vanishing on a neighborhood of $\partial \Omega \backslash \Gamma_{\text {in }}$ and that $\Gamma_{\text {in }}$ is Lipschitz. This justifies the latter integration by parts although $\tilde{U}_{h}^{+}\left(\Gamma_{\text {in }}\right)$ may fail to be Lipschitz.

Now, we insert the identities (3.8-3.9) into (3.7) and let $h \rightarrow 0$. Recalling regularity of $\left(\tilde{\varrho}_{B}, \tilde{\mathbf{u}}_{B}\right)$ evoked in (3.5) and summability of $(\varrho, \mathbf{u})$, we deduce finally that

$$
\begin{gathered}
\int_{\Omega} b(\varrho(\tau)) \varphi(\tau) \mathrm{d} x-\int_{\Omega} b\left(\varrho_{0}\right) \varphi(0) \mathrm{d} x \\
=\int_{0}^{\tau} \int_{\Omega}\left(b(\varrho) \partial_{t} \varphi+b(\varrho) \mathbf{u} \cdot \nabla_{x} \varphi-\varphi\left(b^{\prime}(\varrho) \varrho-b(\varrho)\right) \operatorname{div}_{x} \mathbf{u}\right) \mathrm{d} x \mathrm{~d} t+\int_{0}^{\tau} \int_{\partial \Omega} b\left(\varrho_{B}\right) \mathbf{u}_{B} \cdot \mathbf{n} \varphi \mathrm{d} S_{x} \mathrm{~d} t
\end{gathered}
$$

for all $\tau \in[0, T]$, for any $\varphi \in C_{c}^{1}\left([0, T] \times\left(\Omega \cup \Gamma_{\text {in }}\right)\right)$.

This finishes proof of Lemma 3.1.

\subsection{The case of bounded oscillations defect measure}

If the density is not square integrable, but if it is a weak limit of a sequence whose oscillations defect measure is bounded, one replaces in the case of theory with no inflow/outflow boundary data Lemma 3.1 by another result, see [11]. The goal of this Section is to generalize this result to continuity equation with non homogenous boundary data.

We introduce the $L^{p}$-oscillations defect measure of the sequence $\varrho_{\delta}$ which admits a weak limit $\varrho$ in $L^{1}\left(Q_{T}\right)$ as follows

$$
\operatorname{Osc}_{p}\left[\varrho_{\delta} \rightarrow \varrho\right]\left(Q_{T}\right) \equiv \sup _{k \geq 1}\left(\limsup _{\delta \rightarrow 0} \int_{Q_{T}}\left|T_{k}\left(\varrho_{\delta}\right)-T_{k}(\varrho)\right|^{p} \mathrm{~d} x \mathrm{~d} t\right),
$$

where truncation $T_{k}(\varrho)$ of $\varrho$ is defined as follows

$$
T_{k}(z)=k T(z / k), \quad T \in C^{1}[0, \infty), \quad T(z)=\left\{\begin{array}{c}
z \text { if } z \in[0,1], \\
\text { concave on }[0, \infty) \\
2 \text { if } z \geq 3,
\end{array}\right.
$$

where $k>1$.

The wanted result is the following lemma.

Lemma 3.2. Suppose that $\Omega \subset R^{d}, d=2,3$ is a bounded Lipschitz domain and let $\left(\varrho_{B}, \mathbf{u}_{B}\right)$ satisfy assumptions (2.1). Assume that the inflow portion of the boundary $\Gamma_{\text {in }}$ is a $C^{2}$ open $(d-1)$-dimensional manifold. Suppose further that

$$
\begin{array}{cc}
\varrho_{\delta} \rightarrow \varrho \quad \text { in } L^{p}((0, T) \times \Omega), \quad p>1, & \\
\mathbf{u}_{\delta} \rightarrow \mathbf{u} \quad \text { in } L^{r}\left((0, T) \times \Omega ; R^{d}\right), \\
\nabla \mathbf{u}_{\delta} \rightarrow \nabla \mathbf{u} & \text { in } L^{r}\left((0, T) \times \Omega ; R^{d^{2}}\right), \quad r>1 .
\end{array}
$$


and that

$$
\mathrm{Osc}_{q}\left[\varrho_{\delta} \rightarrow \varrho\right]((0, T) \times \Omega)<\infty
$$

for $\frac{1}{q}<1-\frac{1}{r}$, where $\left(\varrho_{\delta}, \mathbf{u}_{\delta}\right)$ solve the renormalized continuity equation (2.13) (with any $b \in C^{1}[0, \infty)$ and $b^{\prime}$ having compact support). Then the limit functions $\varrho$, $\mathbf{u}$ solve again the renormalized continuity equation (2.13) for any b belonging to the same class.

Remark 3.3. Let $\left\{\Omega_{n}\right\}_{n=1}^{\infty}, \Omega_{n} \subset \Omega_{n+1} \subset \Omega, \bar{\Omega}_{n} \subset \Omega \cup \Gamma_{\text {in }}$ be a family of domains satisfying condition: For all compact $\mathfrak{K}$ of $\Omega \cup \Gamma_{\text {in }}$ there exists $n \in N_{*}$ such that $\mathfrak{K} \subset \bar{\Omega}_{n}$. Then one can replace in Lemma 3.2 assumption (3.12) by a slightly weaker assumption

$\forall n \in N_{*}, \varrho_{\delta} \rightarrow \varrho$ in $L^{p}\left((0, T) \times \Omega_{n}\right)$, where $\varrho \in L^{p}(\Omega), p>1$

$\forall n \in N_{*}, \mathbf{u}_{\delta} \rightarrow \mathbf{u}$ in $L^{r}\left((0, T) \times \Omega_{n} ; R^{d}\right)$, where $\mathbf{u} \in L^{r}\left(\Omega ; R^{d}\right)$,

$\forall n \in N_{*}, \nabla \mathbf{u}_{\delta} \rightarrow \nabla \mathbf{u}$ in $L^{r}\left((0, T) \times \Omega_{n} ; R^{d^{2}}\right)$, where $\nabla \mathbf{u} \in L^{r}\left(\Omega ; R^{d^{2}}\right), r>1$.

This observation (which is seen from a brief inspection of the proof hereafter) is not needed in the present paper but may be of interest whenever one deals with the stability of weak solutions with respect to perturbations of the boundary in the case of non homogenous boundary data.

\section{Proof of Lemma 3.2}

The proof of the lemma follows closely with only minor modifications the similar proof when boundary velocity is zero, see [11]. During the process of the proof one shall need Lemma 3.1. This is the only moment when the requirement on the regularity of $\Gamma_{\text {in }}$ is needed. We present however here the entire proof for the sake of completeness.

Renormalized continuity eqution (2.13) with $b=T_{k}$ reads

$$
\begin{gathered}
\int_{\Omega} T_{k}\left(\varrho_{\delta}\right) \varphi(\tau, x) \mathrm{d} x-\int_{\Omega} T_{k}\left(\varrho_{0}\right) \varphi(0, x) \mathrm{d} x= \\
\int_{0}^{\tau} \int_{\Omega}\left(T_{k}\left(\varrho_{\delta}\right) \partial_{t} \varphi+T_{k}\left(\varrho_{\delta}\right) \mathbf{u}_{\delta} \cdot \nabla_{x} \varphi-\varphi\left(T_{k}^{\prime}\left(\varrho_{\delta}\right) \varrho_{\delta}-T_{k}\left(\varrho_{\delta}\right)\right) \operatorname{div}_{x} \mathbf{u}_{\delta}\right) \mathrm{d} x \mathrm{~d} t-\int_{0}^{\tau} \int_{\Gamma_{\mathrm{in}}} T_{k}\left(\varrho_{B}\right) \mathbf{u}_{B} \cdot \mathbf{n} \varphi \mathrm{d} S_{x} \mathrm{~d} t
\end{gathered}
$$

for any $\varphi \in C^{1}\left([0, T] \times\left(\Omega \cup \Gamma_{\text {in }}\right)\right)$.

Passing to the limit $\delta \rightarrow 0$ in (3.14), we get

$$
\begin{gathered}
\int_{\Omega} \overline{T_{k}(\varrho)} \varphi(\tau, x) \mathrm{d} x-\int_{\Omega} T_{k}\left(\varrho_{0}\right) \varphi(0, x) \mathrm{d} x= \\
\int_{0}^{\tau} \int_{\Omega}\left(\overline{T_{k}(\varrho)} \partial_{t} \varphi+\overline{T_{k}(\varrho)} \mathbf{u} \cdot \nabla_{x} \varphi-\varphi \overline{\left(T_{k}^{\prime}(\varrho) \varrho-T_{k}(\varrho)\right) \operatorname{div}_{x} \mathbf{u}}\right) \mathrm{d} x \mathrm{~d} t-\int_{0}^{\tau} \int_{\Gamma_{\mathrm{in}}} T_{k}\left(\varrho_{B}\right) \mathbf{u}_{B} \cdot \mathbf{n} \varphi \mathrm{d} S_{x} \mathrm{~d} t
\end{gathered}
$$

for any $\varphi \in C^{1}\left([0, T] \times\left(\Omega \cup \Gamma_{\text {in }}\right)\right)$. Since for fixed $k>0, \overline{T_{k}(\varrho)} \in L^{\infty}\left((0, T) \times R^{d}\right)$, we can employ Lemma 3.1 (with a slight obvious modification which takes into account the non zero right hand side $\overline{\left.\left(T_{k}^{\prime}(\varrho) \varrho-T_{k}(\varrho)\right) \operatorname{div}_{x} \mathbf{u}\right)}$ in order to infer that

$$
\int_{\Omega} b_{M}\left(\overline{T_{k}(\varrho)}\right) \varphi(\tau, x) \mathrm{d} x-\int_{\Omega} b_{M}\left(\varrho_{0}\right) \varphi(0, x) \mathrm{d} x=
$$




$$
\begin{gathered}
\int_{0}^{\tau} \int_{\Omega}\left(b_{M}\left(\overline{T_{k}(\varrho)}\right) \partial_{t} \varphi+b_{M}\left(\overline{T_{k}(\varrho)}\right) \mathbf{u} \cdot \nabla_{x} \varphi-\varphi\left(b_{M}^{\prime}\left(\overline{T_{k}(\varrho)}\right) \overline{T_{k}(\varrho)}-b_{M}\left(\overline{T_{k}(\varrho)}\right)\right) \mathrm{div}{ }_{x} \mathbf{u}\right) \mathrm{d} x \mathrm{~d} t \\
\quad+\int_{0}^{\tau} \int_{\Omega} \overline{\left(\varrho T_{k}^{\prime}(\varrho)-\varrho\right) \operatorname{div}_{x} \mathbf{u}} b_{M}^{\prime}\left(\overline{T_{k}(\varrho)}\right) \mathrm{d} x \mathrm{~d} t-\int_{0}^{\tau} \int_{\Gamma_{\mathrm{in}}} b_{M}\left(T_{k}\left(\varrho_{B}\right)\right) \mathbf{u}_{B} \cdot \mathbf{n} \varphi \mathrm{d} S_{x} \mathrm{~d} t
\end{gathered}
$$

holds with any $\varphi \in C^{1}\left([0, T] \times\left(\Omega \cup \Gamma_{\text {in }}\right)\right)$ and any $b_{M} \in C^{1}[0, \infty)$ with $b_{M}^{\prime}$ having a compact support in $[0, M)$.

Seeing that by lower weak semi-continuity of $L^{1}$ norms, $\overline{T_{k}(\varrho)} \rightarrow \varrho$ in $L^{1}((0, T) \times \Omega)$ as $k \rightarrow \infty$, we obtain from equation (3.15) by using the Lebesgue dominated convergence theorem

$$
\begin{gathered}
\int_{\Omega} b_{M}(\varrho) \varphi(\tau, x) \mathrm{d} x-\int_{\Omega} b_{M}\left(\varrho_{0}\right) \varphi(0, x) \mathrm{d} x= \\
\int_{0}^{\tau} \int_{\Omega}\left(b_{M}(\varrho) \partial_{t} \varphi+b_{M}(\varrho) \mathbf{u} \cdot \nabla_{x} \varphi-\varphi\left(b_{M}^{\prime}(\varrho) \varrho-b_{M}(\varrho)\right) \operatorname{div}_{x} \mathbf{u}\right) \mathrm{d} x \mathrm{~d} t-\int_{0}^{\tau} \int_{\Gamma_{\mathrm{in}}} b_{M}\left(\varrho_{B}\right) \mathbf{u}_{B} \cdot \mathbf{n} \varphi \mathrm{d} S_{x} \mathrm{~d} t
\end{gathered}
$$

with any $\varphi \in C^{1}\left([0, T] \times\left(\Omega \cup \Gamma_{\text {in }}\right)\right)$ as $k \rightarrow \infty$, provided we show that

$$
\left\|\overline{\left.\left(\varrho T_{k}^{\prime}(\varrho)-\varrho\right) \operatorname{div}_{x} \mathbf{u}\right)} b_{M}^{\prime}\left(\overline{T_{k}(\varrho)}\right)\right\|_{L^{1}((0, T) \times \Omega)} \rightarrow 0 \text { as } k \rightarrow \infty .
$$

To show the latter relation we use lower weak semicontinuity of $L^{1}$ norm, Hölder's inequality, uniform bound of $\mathbf{u}_{\delta}$ in $L^{r}\left(0, T ; W^{1, r}(\Omega)\right)$ and interpolation of $L^{r^{\prime}}$ between Lebesgue spaces $L^{1}$ and $L^{q}$ to get

$$
\begin{gathered}
\left\|\overline{\left.\left(\varrho T_{k}^{\prime}(\varrho)-\varrho\right) \operatorname{div}_{x} \mathbf{u}\right)} b_{M}^{\prime}\left(\overline{T_{k}(\varrho)}\right)\right\|_{L^{1}((0, T) \times \Omega)} \\
\leq \max _{z \in[0, M]}\left|b_{M}^{\prime}(z)\right| \int_{\left\{\overline{T_{k}(\varrho)} \leq M\right\}}\left|\overline{\left.\left(\varrho T_{k}^{\prime}(\varrho)-\varrho\right) \operatorname{div} \mathbf{u}_{x} \mathbf{u}\right)}\right| \mathrm{d} x \mathrm{~d} t \\
\left.\leq c \sup _{\delta>0} \| \varrho_{\delta} T_{k}^{\prime}\left(\varrho_{\delta}\right)-\varrho_{\delta}\right) \|_{L^{1}((0, T) \times \Omega)}^{\frac{q(r-1)-r}{r(q-1)}} \\
\left.\liminf _{\delta \rightarrow 0} \| \varrho_{\delta} T_{k}^{\prime}\left(\varrho_{\delta}\right)-\varrho_{\delta}\right) \|_{L^{q}\left(\left\{\overline{T_{k}(\varrho)} \leq M\right\}\right)}^{\frac{q}{r(q-1)}}
\end{gathered}
$$

We have

$$
\left.\| \varrho_{\delta} T_{k}^{\prime}\left(\varrho_{\delta}\right)-\varrho_{\delta}\right)\left\|_{L^{1}((0, T) \times \Omega)} \leq 2 \sup _{\delta>0}\right\| \varrho_{\delta} \|_{L^{1}\left(\left\{\varrho_{\delta} \geq k\right\}\right)} \rightarrow 0 \text { as } k \rightarrow \infty
$$

by virtue of the uniform bound of $\varrho_{\delta}$ in $L^{p}((0, T) \times \Omega$ ) (in the above we have also used algebraic relation $\left.z T_{k}^{\prime}(z)-T_{k}(z) \leq 2 z 1_{\{z \geq k\}}\right)$, while

$$
\begin{gathered}
\left.\| \varrho_{\delta} T_{k}^{\prime}\left(\varrho_{\delta}\right)-\varrho_{\delta}\right)\left\|_{L^{q}\left(\left\{\overline{T_{k}(\varrho)} \leq M\right\}\right)} \leq 2\right\| T_{k}\left(\varrho_{\delta}\right) \|_{L^{1}\left(\left\{\overline{T_{k}(\varrho)} \leq M\right\}\right)} \\
\leq 2\left(\left\|T_{k}\left(\varrho_{\delta}\right)-T_{k}(\varrho)\right\|_{L^{q}((0, T) \times \Omega)}+\left\|T_{k}(\varrho)-\overline{T_{k}(\varrho)}\right\|_{L^{q}((0, T) \times \Omega)}+\left\|\overline{T_{k}(\varrho)}\right\|_{L^{q}\left(\left\{\overline{T_{k}(\varrho)} \leq M\right\}\right)}\right)
\end{gathered}
$$

where we have used algebraic relation $z T_{k}^{\prime}(z) \leq 2 T_{k}(z)$ and the Minkowski inequality. Equation (3.16) implies (2.13) with any $b \in C^{1}[0, \infty), b^{\prime}$ with compact support by virtue of the Lebesgue dominated convergence theorem. Lemma 3.2 is proved. 


\section{Approximate problem}

Our goal is to construct solutions the existence of which is claimed in Theorem 2.4. To this end, we adopt the approximation scheme based on pressure regularization (small parameter $\delta>0$ ) adding artificial viscosity terms to both (1.1) and (1.2) (small parameter $\varepsilon>0$ ). This is so far standard procedure. Moreover, mostly for technical reasons, we regularize the momentum equation by adding a convenient dissipative monotone operator with small parameter $\varepsilon>0$. (This step is not needed when treating zero boundary data, but seems to be necessary if the boundary velocity is non zero.) In sharp contrast with [13], we consider for the new system a boundary value problem on $\Omega$ with the non homogenous boundary conditions for velocity and a convenient nonlinear Neumann type boundary condition for density. The approximated problem is determined in Section 4.1 and the crucial theorem on its solvability and estimates is formulated in Section 4.2 (see Lemma 4.1). Lemma 4.1 is proved by a standard combination of a fixed point and Galerkin method, see Section 4.3. After this process we have at our disposal a convenient solution of the approximate problem. Once the sequence of approximated solutions is available the limit process has to be effectuated in order 1 . $\varepsilon \rightarrow 0$ (see Section 5 ), 2. $\delta \rightarrow 0$ (see Section 6).

\subsection{Approximating system of equations}

The approximate problem reads:

$$
\begin{gathered}
\partial_{t} \varrho-\varepsilon \Delta_{x} \varrho+\operatorname{div}_{x}(\varrho \mathbf{u})=0, \\
\varrho(0, x)=\varrho_{0}(x),\left.\left(-\varepsilon \nabla_{x} \varrho+\varrho \mathbf{u}\right) \cdot \mathbf{n}\right|_{I \times \partial \Omega}=\left\{\begin{array}{l}
\varrho_{B} \mathbf{u}_{B} \cdot \mathbf{n} \text { if }\left[\mathbf{u}_{B} \cdot \mathbf{n}\right](x) \leq 0, x \in \partial \Omega, \\
\varrho \mathbf{u}_{B} \cdot \mathbf{n} \text { if }\left[\mathbf{u}_{B} \cdot \mathbf{n}\right](x)>0, x \in \partial \Omega
\end{array}\right. \\
\partial_{t}(\varrho \mathbf{u})+\operatorname{div}_{x}(\varrho \mathbf{u} \otimes \mathbf{u})+\nabla_{x} p_{\delta}(\varrho)=\operatorname{div}_{x} \mathbb{S}\left(\nabla_{x} \mathbf{u}\right)-\varepsilon \nabla_{x} \varrho \cdot \nabla_{x} \mathbf{u}+\varepsilon \operatorname{div}\left(\left|\nabla_{x}\left(\mathbf{u}-\mathbf{u}_{\infty}\right)\right|^{2} \nabla_{x}\left(\mathbf{u}-\mathbf{u}_{\infty}\right)\right) \\
\mathbf{u}(0, x)=\mathbf{u}_{0}(x),\left.\mathbf{u}\right|_{I \times \partial \Omega}=\mathbf{u}_{B},
\end{gathered}
$$

with positive parameters $\varepsilon>0, \delta>0$, where we have denoted

$$
p_{\delta}(\varrho)=p(\varrho)+\delta \varrho^{\beta}, \quad \beta>\max \{\gamma, 9 / 2\}
$$

and where $\mathbf{u}_{\infty}$ is an extension of $\mathbf{u}_{B}$ from Lemma 2.2. The exact choice of $\beta$ is irrelevant from the point of view of the final result provided it is sufficiently large. It is guided by convenience in proofs; it might not be optimal.

Anticipating the future development, we denote:

$$
\mathscr{H}_{\delta}(\varrho)=\mathscr{H}(\varrho)+\delta H^{(\beta)}(\varrho), H_{\delta}(\varrho)=H(\varrho)+\delta H^{(\beta)}(\varrho), H^{(\beta)}(\varrho)=\varrho \int_{1}^{\varrho} z^{\beta-2} \mathrm{~d} z=\frac{1}{\beta-1} \varrho^{\beta}
$$

and

$$
\begin{gathered}
\mathscr{E}_{\delta}(\varrho \mid r)=\mathscr{E}(\varrho \mid r)+\delta E^{(\beta)}(\varrho \mid r), \quad E_{\delta}(\varrho \mid r)=E(\varrho \mid r)+\delta E^{(\beta)}(\varrho \mid r), \\
E^{(\beta)}(\varrho \mid r)=H^{(\beta)}(\varrho)-\left[H^{(\beta)}\right]^{\prime}(r)(\varrho-r)-H^{(\beta)}(r) .
\end{gathered}
$$




\subsection{Generalized solutions of the approximate problem}

Definition 4.1 A couple $\left(\varrho_{\varepsilon}, \mathbf{u}_{\varepsilon}\right)$ and associated tensor field $\mathbb{Z}_{\varepsilon}$ is a generalized solution of the sequence of problems $(4 \cdot 1-4.4)_{\varepsilon>0}$ iff the following holds:

1. It belongs to the functional spaces:

$$
\begin{gathered}
\varrho_{\varepsilon} \in L^{\infty}\left(0, T ; L^{\beta}(\Omega)\right) \cap L^{2}\left(0, T ; W^{1,2}(\Omega)\right), 0 \leq \varrho_{\varepsilon} \text { a.a. in }(0, T) \times \Omega \\
\mathbf{u}_{\varepsilon} \in L^{2}\left(0, T ; W^{1,2}\left(\Omega ; R^{3}\right)\right) \cap L^{4}\left(0, T ; W^{1,4}\left(\Omega ; R^{3}\right)\right),\left.\mathbf{u}_{\varepsilon}\right|_{I \times \partial \Omega}=\mathbf{u}_{B} \\
\mathbb{Z}_{\varepsilon} \rightarrow 0 \text { in } L^{4 / 3}\left(Q_{T} ; R^{3}\right) \text { as } \varepsilon \rightarrow 0 .
\end{gathered}
$$

2. Function $\varrho_{\varepsilon} \in C_{\text {weak }}\left([0, T], L^{\beta}(\Omega)\right)$ and the integral identity

$$
\begin{gathered}
\int_{\Omega} \varrho_{\varepsilon}(\tau, x) \varphi(\tau, x) \mathrm{d} x-\int_{\Omega} \varrho_{0}(x) \varphi(0, x) \mathrm{d} x= \\
\int_{0}^{\tau} \int_{\Omega}\left(\varrho_{\varepsilon} \partial_{t} \varphi+\varrho_{\varepsilon} \mathbf{u}_{\varepsilon} \cdot \nabla_{x} \varphi-\varepsilon \nabla_{x} \varrho_{\varepsilon} \cdot \nabla_{x} \varphi\right) \mathrm{d} x \mathrm{~d} t-\int_{0}^{\tau} \int_{\Gamma_{\mathrm{in}}} \varrho_{B} \mathbf{u}_{B} \cdot \mathbf{n} \varphi \mathrm{d} S_{x} \mathrm{~d} t
\end{gathered}
$$

holds for any $\tau \in[0, T]$ and $\varphi \in C_{c}^{1}\left([0, T] \times\left(\Omega \cup \Gamma_{\text {in }}\right)\right)$;

3. Function $\varrho_{\varepsilon} \mathbf{u}_{\varepsilon} \in C_{\text {weak }}\left([0, T], L^{\frac{2 \beta}{\beta+1}}\left(\Omega ; R^{3}\right)\right)$, and the integral identity

$$
\begin{gathered}
\int_{\Omega} \varrho_{\varepsilon} \mathbf{u}_{\varepsilon}(\tau, \cdot) \cdot \boldsymbol{\varphi}(\tau, \cdot) \mathrm{d} x-\int_{\Omega} \varrho_{0} \mathbf{u}_{0}(\cdot) \boldsymbol{\varphi}(0, \cdot) \mathrm{d} x=-\int_{0}^{\tau} \int_{\Omega} \mathbb{Z}_{\varepsilon}: \nabla_{x} \varphi \mathrm{d} x \mathrm{~d} t \\
+\int_{0}^{\tau} \int_{\Omega}\left(\varrho_{\varepsilon} \mathbf{u}_{\varepsilon} \partial_{t} \varphi+\varrho_{\varepsilon} \mathbf{u}_{\varepsilon} \otimes \mathbf{u}_{\varepsilon}: \nabla_{x} \boldsymbol{\varphi}+p_{\delta}\left(\varrho_{\varepsilon}\right) \operatorname{div}_{x} \boldsymbol{\varphi}-\varepsilon \nabla_{x} \varrho_{\varepsilon} \cdot \nabla_{x} \mathbf{u}_{\varepsilon} \cdot \boldsymbol{\varphi}-\mathbb{S}\left(\nabla_{x} \mathbf{u}_{\varepsilon}\right): \nabla_{x} \boldsymbol{\varphi}\right) \mathrm{d} x \mathrm{~d} t
\end{gathered}
$$

holds for any $\tau \in[0, T]$ and any $\varphi \in C_{c}^{1}\left([0, T] \times \Omega ; R^{3}\right)$.

4. Energy inequality

$$
\begin{gathered}
\int_{\Omega}\left(\frac{1}{2} \varrho_{\varepsilon}\left|\mathbf{u}_{\varepsilon}-\mathbf{u}_{\infty}\right|^{2}+H_{\delta}\left(\varrho_{\varepsilon}\right)+\frac{\Sigma}{2} \varrho_{\varepsilon}^{2}\right)(\tau) \mathrm{d} x \\
+\delta \frac{\beta-1}{2} \int_{0}^{\tau} \int_{\Gamma_{\mathrm{in}}} \varrho_{\varepsilon}^{\beta}\left|\mathbf{u}_{B} \cdot \mathbf{n}\right| \mathrm{d} S_{x} \mathrm{~d} t+\delta \frac{1}{\beta-1} \int_{0}^{\tau} \int_{\Gamma_{\text {out }}} \varrho_{\varepsilon}^{\beta}\left|\mathbf{u}_{B} \cdot \mathbf{n}\right| \mathrm{d} S_{x} \mathrm{~d} t+\frac{\Sigma}{2} \int_{0}^{\tau} \int_{\Gamma_{\mathrm{in}}} \varrho_{\varepsilon}^{2}\left|\mathbf{u}_{B} \cdot \mathbf{n}\right| \mathrm{d} S_{x} \mathrm{~d} t \\
+\int_{0}^{\tau} \int_{\Omega}\left(\mathbb{S}\left(\nabla_{x}\left(\mathbf{u}_{\varepsilon}-\mathbf{u}_{\infty}\right)\right): \nabla_{x}\left(\mathbf{u}_{\varepsilon}-\mathbf{u}_{\infty}\right)+\varepsilon H_{\delta}^{\prime \prime}\left(\varrho_{\varepsilon}\right)\left|\nabla_{x} \varrho_{\varepsilon}\right|^{2}+\varepsilon \Sigma\left|\nabla_{x} \varrho_{\varepsilon}\right|^{2}+\varepsilon\left|\nabla_{x}\left(\mathbf{u}_{\varepsilon}-\mathbf{u}_{\infty}\right)\right|^{4}\right) \mathrm{d} x \mathrm{~d} t \\
\leq \int_{\Omega}\left(\frac{1}{2} \varrho_{0}\left|\mathbf{u}_{0}-\mathbf{u}_{\infty}\right|^{2}+H_{\delta}\left(\varrho_{0}\right)+\frac{\Sigma}{2} \varrho_{0}^{2}\right) \mathrm{d} x \\
-\left(\underline{\underline{E}-\delta B)} \int_{0}^{\tau} \int_{\Gamma_{\text {in }}}\left|\mathbf{u}_{B} \cdot \mathbf{n}\right| \mathrm{d} S_{x} \mathrm{~d} t-(\underline{H}-\delta A) \int_{0}^{\tau} \int_{\Gamma_{\text {out }}}\left|\mathbf{u}_{B} \cdot \mathbf{n}\right| \mathrm{d} S_{x} \mathrm{~d} t\right.
\end{gathered}
$$




$$
\begin{gathered}
-\int_{0}^{\tau} \int_{\Gamma_{\mathrm{in}}} H_{\delta}\left(\varrho_{B}\right) \mathbf{u}_{B} \cdot \mathbf{n d} S_{x} \mathrm{~d} t+\Sigma \int_{0}^{\tau} \int_{\Gamma_{\mathrm{in}}} \varrho_{\varepsilon} \varrho_{B}\left|\mathbf{u}_{B} \cdot \mathbf{n}\right| \mathrm{d} S_{x} \mathrm{~d} t \\
-\int_{0}^{\tau} \int_{\Omega} p_{\delta}\left(\varrho_{\varepsilon}\right) \mathrm{div} \mathbf{u}_{\infty} \mathrm{d} x \mathrm{~d} t-\int_{0}^{\tau} \int_{\Omega} \varrho_{\varepsilon} \mathbf{u}_{\varepsilon} \cdot \nabla_{x} \mathbf{u}_{\infty} \cdot\left(\mathbf{u}_{\varepsilon}-\mathbf{u}_{\infty}\right) \mathrm{d} x \mathrm{~d} t \\
+\varepsilon \int_{0}^{\tau} \int_{\Omega} \nabla_{x} \varrho_{\varepsilon} \cdot \nabla_{x}\left(\mathbf{u}_{\varepsilon}-\mathbf{u}_{\infty}\right) \cdot \mathbf{u}_{\infty} \mathrm{d} x \mathrm{~d} t-\int_{0}^{\tau} \int_{\Omega} \mathbb{S}\left(\nabla_{x} \mathbf{u}_{\infty}\right): \nabla_{x}\left(\mathbf{u}_{\varepsilon}-\mathbf{u}_{\infty}\right) \mathrm{d} x \mathrm{~d} t \\
-\frac{\Sigma}{2} \int_{0}^{\tau} \int_{\Omega} \varrho_{\varepsilon}^{2} \operatorname{div} \mathbf{u}_{\varepsilon} \mathrm{d} x \mathrm{~d} t, \quad A=\frac{1}{\beta-1}, B=\frac{2^{\beta-1}}{\beta-1} \bar{\varrho}_{B}^{\beta}
\end{gathered}
$$

holds for a.a. $\tau \in(0, T)$, any $\Sigma>0$ with some continuous extension $\mathbf{u}_{\infty}$ of $\mathbf{u}_{B}$ in the class (2.11). In the above, $H_{\delta}$ is defined in (4.6), $\underline{H}$ is defined in (2.10) and

$$
-\infty<\underline{\mathfrak{E}}:=\inf _{\varrho>0, \underline{\varrho}_{B}<r<\bar{\varrho}_{B}} \mathfrak{E}(r \mid \varrho) \text {, where } \mathfrak{E}(\cdot \mid \cdot) \text { is defined in (2.4). }
$$

The main achievement of this section is existence theorem for approximating problem (4.1-4.4). It is announced in the next lemma.

Lemma 4.1. Let $\Omega$ be a domain of class $C^{2}$. Let $\left(\varrho_{B}, \mathbf{u}_{B}\right)$ verify assumptions (2.1) and let initial and boundary data verify

$$
\begin{gathered}
\mathbf{u}_{0} \in L^{2}(\Omega), \quad \varrho_{0} \in W^{1,2}(\Omega), 0<\underline{\varrho} \leq \varrho_{0} \leq \bar{\varrho}<\infty . \\
0<\underline{\varrho}_{B} \leq \varrho_{B} \leq \bar{\varrho}_{B}<\infty
\end{gathered}
$$

Then for any continuous extension $\mathbf{u}_{\infty}$ of $\mathbf{u}_{B}$ in class (2.11) there exists a generalized solution $\left(\varrho_{\epsilon}, \mathbf{u}_{\epsilon}\right)$ and $\mathbb{Z}_{\varepsilon}$ to the sequence of approximate problems $(4.1-4.4)_{\varepsilon \in(0,1)}$ - which belongs to functional spaces (4.8), satisfies weak formulations (4.9-4.10) and verifies energy inequality (4.11) - with the following extra properties:

(i) In addition to (4.8) it belongs to functional spaces:

$$
\varrho_{\varepsilon} \in L^{\frac{5}{3} \beta}\left(Q_{T}\right), \sqrt{\varrho_{\varepsilon}}, \varrho_{\epsilon}^{\frac{\beta}{2}} \in L^{2}\left(I, W^{1,2}(\Omega)\right), \partial_{t} \varrho_{\epsilon} \in L^{4 / 3}\left(Q_{T}\right), \nabla^{2} \varrho_{\epsilon} \in L^{4 / 3}\left(Q_{T}\right) .
$$

(ii) In addition to the weak formulation (4.9), the couple $\left(\varrho_{\varepsilon}, \mathbf{u}_{\varepsilon}\right)$ satisfies equation (4.9) in the strong sense, meaning it verifies equation (4.1) with $\left(\varrho_{\varepsilon}, \mathbf{u}_{\varepsilon}\right)$ a.e. in $Q_{T}$, boundary identity (4.2) with $\left(\varrho_{\varepsilon}, \mathbf{u}_{\varepsilon}\right)$ a.e. in $(0, T) \times \partial \Omega$ and initial conditions in the sense $\lim _{t \rightarrow 0+}\left\|\varrho_{\varepsilon}(t)-\varrho_{0}\right\|_{L^{4 / 3}(\Omega)}=0$.

(iii) The couple $\left(\varrho_{\varepsilon}, \mathbf{u}_{\varepsilon}\right)$ satisfies identity

$$
\partial_{t} b\left(\varrho_{\varepsilon}\right)+\varepsilon b^{\prime \prime}\left(\varrho_{\varepsilon}\right)\left|\nabla_{x} \varrho_{\varepsilon}\right|^{2}-\varepsilon \operatorname{div}_{x}\left(b^{\prime}\left(\varrho_{\varepsilon}\right) \nabla_{x} \varrho_{\varepsilon}\right)+\operatorname{div}_{x}\left(b\left(\varrho_{\varepsilon}\right) \mathbf{u}_{\varepsilon}\right)+\left[b^{\prime}\left(\varrho_{\varepsilon}\right) \varrho_{\varepsilon}-b\left(\varrho_{\varepsilon}\right)\right] \operatorname{div}_{x} \mathbf{u}_{\varepsilon}=0
$$

a.e. in $(0, T) \times \Omega$ with any $b \in C^{2}[0, \infty)$, where the space-time derivatives have to be understood in the sense a.e. 
Remark 4.2. Identity (4.16) holds in the weak sense

$$
\begin{gathered}
\int_{\Omega} b\left(\varrho_{\varepsilon}(\tau)\right) \varphi(\tau) \mathrm{d} x-\int_{\Omega} b\left(\varrho_{0}\right) \varphi(0) \mathrm{d} x=\int_{0}^{\tau} \int_{\partial \Omega}\left(\varepsilon b^{\prime}\left(\varrho_{\varepsilon}\right) \nabla_{x} \varrho_{\varepsilon}-b\left(\varrho_{\varepsilon}\right) \mathbf{u}_{\varepsilon}\right) \cdot \mathbf{n} \mathrm{d} S_{x} \mathrm{~d} t \\
+\int_{0}^{\tau} \int_{\Omega}\left[b\left(\varrho_{\varepsilon}\right) \partial_{t} \varphi+\left(b\left(\varrho_{\varepsilon}\right) \mathbf{u}_{\varepsilon}-\varepsilon b^{\prime}\left(\varrho_{\varepsilon}\right) \nabla_{x} \varrho_{\varepsilon}\right) \cdot \nabla_{x} \varphi-\varphi\left(\varepsilon b^{\prime \prime}\left(\varrho_{\varepsilon}\right)\left|\nabla_{x} \varrho_{\varepsilon}\right|^{2}+\left(\varrho_{\varepsilon} b^{\prime}\left(\varrho_{\varepsilon}\right)-b\left(\varrho_{\varepsilon}\right)\right) \operatorname{div} \mathbf{u}_{\varepsilon}\right)\right] \mathrm{d} x \mathrm{~d} t
\end{gathered}
$$

with any $\tau \in[0, T]$ and $\varphi \in C_{c}^{1}([0, T] \times \bar{\Omega})$ with any $b$ whose growth (and that one of its derivatives) in combination with $(4.15)$ guarantees $b\left(\varrho_{\varepsilon}\right) \in C_{\text {weak }}\left([0, T] ; L^{1}(\Omega)\right)$, existence of traces and integrability of all terms appearing at the r.h.s.

\subsection{Solvability of the approximating equations}

This section is devoted to the proof of Lemma 4.1. We adopt the nowadays standard procedure based on computing the approximate density $\varrho$ in terms of $\mathbf{u}$ in (4.1), (4.2), calculating $\mathbf{u}$ via a Galerkin approximation, and applying a fixed point argument, see [17, Chapter 7]. We proceed in several steps. These steps are described in the following subsections.

\subsubsection{Construction of a (strong) solution of problem (4.1-4.2)}

Here we consider the problem (4.1-4.2), with fixed $\varepsilon>0$ and with fixed sufficiently regular $\mathbf{u}$. We may suppose without loss of generality that

$$
\left\{\begin{array}{l}
\mathbf{u}_{B} \cdot \mathbf{n} \text { on } \Gamma_{\text {in }}, \\
0 \text { on } \partial \Omega \backslash \Gamma_{\text {in }}
\end{array}\right\} \equiv v \in C^{1}(\partial \Omega), \quad \varrho_{B} v \equiv g \in C^{1}(\partial \Omega) .
$$

Now, problem (4.1-4.2) may be rewritten as parabolic problem:

$$
\begin{gathered}
\partial_{t} \varrho-\varepsilon \Delta \varrho+\operatorname{div}(\varrho \mathbf{u})=0 \text { in }(0, T) \times \Omega, \\
-\varepsilon \nabla \varrho \cdot \mathbf{n}+\varrho v=g \text { in }(0, T) \times \partial \Omega, \quad \varrho(0)=\varrho_{0} \text { in } \Omega .
\end{gathered}
$$

Applying to problem (4.18) the maximal regularity theory for parabolic systems, we get in particular:

Lemma 4.3. Suppose that $\Omega$ is a bounded domain of class $C^{2}$ and assume further that $\varrho_{0} \in W^{1,2}(\Omega)$, $\mathbf{u} \in L^{\infty}\left(0, T ; W^{1, \infty}(\Omega)\right),\left.\mathbf{u}\right|_{(0, T) \times \partial \Omega}=\mathbf{u}_{B}, v, g \in C^{1}(\partial \Omega)$ are given by (4.17). Then we have:

1. The parabolic problem (4.18) admits a unique solution in the class

$$
\varrho \in L^{2}\left(0, T ; W^{2,2}(\Omega)\right) \cap W^{1,2}\left(0, T ; L^{2}(\Omega)\right) .
$$

2. The following estimates hold: There is $c=c\left(T, \varepsilon,|\Omega|,|\partial \Omega|_{2}, K\right)>0$ (independent of $\mathbf{u}, \varrho_{0}, \varrho_{B}, \mathbf{u}_{B}$ ) such that

$$
\|\varrho(\tau)\|_{L^{2}(\Omega)}^{2} \leq c\left(\left\|\varrho_{0}\right\|_{L^{2}(\Omega)}^{2}+\int_{0}^{\tau} \int_{\partial \Omega} \varrho_{B}^{2}|v| \mathrm{d} S_{x} \mathrm{~d} t\right)
$$




$$
\int_{0}^{\tau}\left\|\nabla_{x} \varrho\right\|_{L^{2}(\Omega)}^{2} \mathrm{~d} t+\int_{0}^{\tau} \int_{\partial \Omega} \varrho^{2}|v| \mathrm{d} S \mathrm{~d} t \leq c\left(\left\|\varrho_{0}\right\|_{L^{2}(\Omega)}^{2}+\int_{0}^{\tau} \int_{\partial \Omega} \varrho_{B}^{2}|v| \mathrm{d} S \mathrm{~d} t\right),
$$

for all $\tau \in[0, T]$, provided

$$
\|\mathbf{u}\|_{L^{\infty}\left(Q_{T}\right)}+\|\operatorname{div} \mathbf{u}\|_{L^{\infty}\left(Q_{T}\right)} \leq K
$$

2. Let

$$
\underline{\varrho} \leq \varrho_{0}(x) \leq \bar{\varrho} \text { for } a . \text { a. } x \in \Omega, \quad \underline{\varrho} \leq \varrho_{B}(x) \leq \bar{\varrho} \text { for all } x \in \Gamma_{\text {in }} .
$$

Then

$$
\underline{\varrho} \exp \left(-\int_{0}^{\tau}\|\operatorname{div} \mathbf{u}(s)\|_{L^{\infty}(\Omega)} \mathrm{d} s\right) \leq \varrho(\tau, x) \leq \bar{\varrho} \exp \left(\int_{0}^{\tau}\|\operatorname{div} \mathbf{u}(s)\|_{L^{\infty}(\Omega)} \mathrm{d} s\right),
$$

in particular,

$$
\underline{\varrho} e^{-K \tau} \leq \varrho(t, x) \leq \bar{\varrho} e^{K \tau}
$$

for all $\tau \in[0, T]$ provided $\mathbf{u}$ verifies condition (4.21). (Here and in the sequel $|A|$ is a Lebesgue measure of set $A \subset R^{3}$ while $|A|_{2}$ denotes its $2-D$ Hausdorf measure.)

3. In the sequel we denote $\varrho=S_{\varrho_{0}, \varrho_{B}, \mathbf{u}_{B}}(\mathbf{u}) \equiv S(\mathbf{u})$. Then

$$
\left\|\left[S_{\varrho_{0}, \varrho_{B}, \mathbf{u}_{B}}\left(\mathbf{u}_{1}\right)-S_{\varrho_{0}, \varrho_{B}, \mathbf{u}_{B}}\left(\mathbf{u}_{2}\right)\right](\tau)\right\|_{L^{2}(\Omega)} \leq \Gamma\left\|\mathbf{u}_{1}-\mathbf{u}_{2}\right\|_{L^{\infty}\left(0, \tau ; W^{1, \infty}(\Omega)\right)}
$$

with some number $\Gamma=\Gamma\left(T, \varepsilon,|\Omega|,|\partial \Omega|_{2}, K,\left\|\varrho_{0}\right\|_{L^{2}(\Omega)},\left\|\varrho_{B}^{2} v\right\|_{L^{1}\left(0 ; T ; L^{1}(\partial \Omega)\right)}\right)>0$, provided both $\mathbf{u}_{1}$, $\mathbf{u}_{2}$ verify (4.21).

\section{Proof of Lemma 4.3}

Proof of statement 1. The parabolic boundary value problem (with elliptic differential operator $\mathscr{A}=$ $-\varepsilon \Delta+\mathbf{u} \cdot \nabla_{x}+\operatorname{divu}$ on $\Omega$ and boundary operator $\mathscr{B}=-\varepsilon \mathbf{n} \cdot \nabla_{x}+v$ on the boundary $\partial \Omega$ ) satisfies all assumptions of the maximal regularity theorem by Denk, Hieber, Prüss [4, Theorem 2.1] with $p=2$ (the coefficients are sufficiently regular in order to verify conditions [4, conditions (SD), (SB)], principal part of operator $\mathscr{A}$ is (normally) elliptic, see [4, condition (E)], while the principal parts of operators $\mathscr{A}$ and $\mathscr{B}$ verify the Shapiro-Lopatinskii conditions [4, condition (LS)], and the data $\varrho_{0}$ and $g$ verify conditions [4, condition (D)], and eventually Bergh and Löfström [3, Theorem 6.4.4] for the identification of the Sobolev space $W^{1,2}(\Omega)$ with the Besov space $B_{2,2}^{1}(\Omega)$ ). Under these circumstances, Theorem 2.1 in [4] yields the statement in item 1. of Lemma 4.3, in particular (4.19). The reader wishing to read more about the maximal regularity to parabolic equations is referred to the monograph [5].

Proof of statement 2. Estimates (4.20) is obtained testing (4.18) by $\varrho$ and using first the Hölder and Young inequalities and next the Gronwall inequality. Indeed, this testing gives

$$
\begin{gathered}
\frac{1}{2} \int_{\Omega} \varrho^{2}(\tau) \mathrm{d} x-\int_{0}^{\tau} \int_{\partial \Omega} \varrho^{2} v \mathrm{~d} S_{x} \mathrm{~d} t+\varepsilon \int_{0}^{\tau} \int_{\Omega}\left|\nabla_{x} \varrho\right|^{2} \mathrm{~d} x \mathrm{~d} t \\
=\frac{1}{2} \int_{\Omega} \varrho_{0}^{2} \mathrm{~d} x-\int_{0}^{\tau} \int_{\partial \Omega} \varrho \varrho_{B} v \mathrm{~d} S_{x} \mathrm{~d} t-\int_{0}^{\tau} \int_{\Omega}\left(\nabla_{x} \varrho \cdot \mathbf{u} \varrho+\varrho^{2} \operatorname{div} \mathbf{u}\right) \mathrm{d} x \mathrm{~d} t,
\end{gathered}
$$


where

$$
\left|\frac{1}{2} \int_{0}^{\tau} \int_{\partial \Omega} \varrho \varrho_{B} v \mathrm{~d} S_{x} \mathrm{~d} t\right| \leq \frac{1}{2} \int_{0}^{\tau} \int_{\partial \Omega} \varrho^{2}|v| \mathrm{d} S_{x} \mathrm{~d} t+\frac{1}{2} \int_{0}^{\tau} \int_{\partial \Omega} \varrho_{B}^{2}|v| \mathrm{d} S_{x} \mathrm{~d} t
$$

and

$\left|\int_{0}^{\tau} \int_{\Omega}\left(\nabla_{x} \varrho \cdot \mathbf{u} \varrho+\varrho^{2} \operatorname{div} \mathbf{u}\right) \mathrm{d} x \mathrm{~d} t\right| \leq \frac{\varepsilon}{2} \int_{0}^{\tau} \int_{\Omega}\left|\nabla_{x} \varrho\right|^{2} \mathrm{~d} x \mathrm{~d} t+\int_{0}^{\tau}\left(\frac{1}{\varepsilon}\|\mathbf{u}\|_{L^{\infty}(\Omega)}^{2}+\|\operatorname{div} \mathbf{u}\|_{L^{\infty}(\Omega)}\right) \int_{\Omega} \varrho^{2} \mathrm{~d} x \mathrm{~d} t ;$

whence application of the standard Gronwall inequality yields

$$
\|\varrho(\tau)\|_{L^{2}(\Omega)}^{2} \leq\left(\left\|\varrho_{0}\right\|_{L^{2}(\Omega)}^{2}+\int_{0}^{\tau} \int_{\partial \Omega} \varrho_{B}^{2}|v| \mathrm{d} S_{x} \mathrm{~d} t\right) \exp \left(\left(K+\frac{1}{\varepsilon} K^{2}\right) \tau\right)
$$

which is first inequality in (4.20). Once this inequality is known, the second inequality is immediate.

Proof of statement 3. We shall proceed to the proof of upper bound (4.22). To this end we define

$$
R(t)=\bar{\varrho} \exp \int_{0}^{t}\|\operatorname{div} \mathbf{u}(s)\|_{L^{\infty}(\Omega)} \mathrm{d} s \text {, i.e } \partial_{t} R+\operatorname{div}(R \mathbf{u}) \geq 0, R(0)=\bar{\varrho} .
$$

We further set $\omega(t, x)=\varrho(t, x)-R(t)$, so that $\omega$ satisfies

$$
\partial_{t} \omega-\varepsilon \Delta \omega+\operatorname{div}(\omega \mathbf{u}) \leq 0, \text { in }(0, T) \times \Omega, \quad-\varepsilon \partial \omega \cdot \mathbf{n}+\omega v=\left(\varrho_{B}-R\right) v \text { in }(0, T) \times \partial \Omega .
$$

Testing the latter inequality by $\omega^{+}$(the positive part of $\omega$ ) we get while reasoning in the same way as in the proof of estimate (4.20),

$$
\begin{gathered}
\frac{1}{2} \int_{\Omega}\left|\omega^{+}\right|^{2}(\tau) \mathrm{d} x+\int_{0}^{\tau} \int_{\partial \Omega}\left|\omega^{+}\right|^{2}|v| \mathrm{d} S_{x} \mathrm{~d} t+\varepsilon \int_{0}^{\tau} \int_{\Omega}\left|\nabla_{x} \omega^{+}\right|^{2} \mathrm{~d} x \mathrm{~d} t \\
\leq \frac{1}{2} \int_{\Omega}\left|\omega^{+}\right|^{2}(0) \mathrm{d} x+\int_{0}^{\tau} \int_{\partial \Omega} \omega^{+}\left(\varrho_{B}-R\right)|v| \mathrm{d} S_{x} \mathrm{~d} t-\int_{0}^{\tau} \int_{\Omega}\left(\nabla_{x} \omega^{+} \cdot \mathbf{u} \omega^{+}+\left|\omega^{+}\right|^{2} \operatorname{div} \mathbf{u}\right) \mathrm{d} x \mathrm{~d} t .
\end{gathered}
$$

Now we employ the fact that the first term at the right hand side is zero, while the second term is non positive, and handle the last term as in the proof of (4.20) to finally get $\left\|\omega^{+}(\tau)\right\|_{L^{2}(\Omega)}=0$ which yields the upper bound in (4.22). To derive the lower bound we shall repeat the same procedure with

$$
R(t)=\varrho \underline{e x p}\left(-\int_{0}^{t}\|\operatorname{div} \mathbf{u}(s)\|_{L^{\infty}(\Omega)} \mathrm{d} s\right) \text { and } \omega(t, x)=R(t)-\varrho(t, x) .
$$

Proof of statement 4. The difference $\eta \equiv \varrho_{1}-\varrho_{2}$, where $\varrho_{i}=S_{\varrho_{0}, \varrho_{B}, \mathbf{u}_{B}}\left(\mathbf{u}_{i}\right)$, verifies equation:

$$
\partial_{t} \eta-\varepsilon \Delta \eta=F \text { a.e. in } Q_{T}, \quad-\varepsilon \nabla_{x} \eta \cdot \mathbf{n}+\eta v=0 \text { in }(0, T) \times \partial \Omega
$$


with zero initial data, where $F=-\varrho_{1} \operatorname{div}\left(\mathbf{u}_{1}-\mathbf{u}_{2}\right)-\nabla_{x} \varrho_{1} \cdot\left(\mathbf{u}_{1}-\mathbf{u}_{2}\right)-\eta \operatorname{div} \mathbf{u}_{2}-\nabla_{x} \eta \cdot \mathbf{u}_{2}$,

$$
\left|\int_{\Omega} F \eta \mathrm{d} x\right| \leq \frac{1}{2}\left\|\mathbf{u}_{1}-\mathbf{u}_{2}\right\|_{W^{1, \infty}(\Omega)}^{2}+\left(\frac{1}{2}\left\|\varrho_{1}\right\|_{W^{1,2}(\Omega)}^{2}+K+\frac{1}{\varepsilon} K^{2}\right)\|\eta\|_{L^{2}(\Omega)}^{2}+\frac{\varepsilon}{2}\left\|\nabla_{x} \eta\right\|_{L^{2}(\Omega)}^{2} .
$$

Therefore, by the same token as before, after testing the above equation by $\eta$, we get

$$
\begin{gathered}
\|\eta(\tau)\|_{L^{2}(\Omega)}^{2}+\frac{\varepsilon}{2} \int_{0}^{\tau}\left\|\nabla_{x} \eta\right\|_{L^{2}(\Omega)}^{2} \mathrm{~d} t+\int_{0}^{\tau} \int_{\partial \Omega} \eta^{2}|v| \mathrm{d} S_{x} \mathrm{~d} t \\
\leq \frac{\tau}{2}\left\|\mathbf{u}_{1}-\mathbf{u}_{2}\right\|_{W^{1, \infty}\left(Q_{\tau}\right)}^{2}+\int_{0}^{\tau}\left(\frac{1}{2}\left\|\varrho_{1}\right\|_{W^{1,2}(\Omega)}^{2}+K+\frac{1}{\varepsilon} K^{2}\right)\|\eta\|_{L^{2}(\Omega)}^{2} \mathrm{~d} t
\end{gathered}
$$

whence Gronwall lemma and bounds (4.20) yield the desired result (4.23).

\subsubsection{Galerkin approximation for approximate problem (4.1-4.4)}

We start by introducing notations and gathering some preliminary material:

1. We denote

$$
X=\operatorname{span}\left\{\Phi_{i}\right\}_{i=1}^{N} \text { where } \mathscr{B}:=\left\{\Phi_{i} \in C_{c}^{\infty}(\Omega) \mid i \in \mathbb{N}^{*}\right\} \text { is an orthonormal basis in } L^{2}\left(\Omega ; R^{3}\right)
$$

a finite dimensional real Hilbert space with scalar product $(\cdot, \cdot)_{X}$ induced by the scalar product in $L^{2}\left(\Omega ; R^{3}\right)$ and $\|\cdot\|_{X}$ the norm induced by this scalar product. We denote by $P_{N}$ the orthogonal projection of $L^{2}\left(\Omega ; R^{3}\right)$ to $X$. Since $X$ is finite-dimensional, norms on $X$ induced by $W^{k, p}$-norms, $k \in N, 1 \leq p \leq \infty$ are equivalent. In particular, there are universal numbers (depending solely on the dimension $N$ of $X) 0<\underline{d}<\bar{d}<\infty$ such that

$$
\underline{d}\|\mathbf{v}\|_{W^{1, \infty}(\Omega)} \leq\|\mathbf{v}\|_{X} \equiv\|\mathbf{v}\|_{L^{2}(\Omega)} \leq \bar{d}\|\mathbf{v}\|_{W^{1, \infty}(\Omega)}, \text { for all } \mathbf{v} \in X .
$$

2. Let $g \in L^{1}\left(Q_{T}\right)$, infess $(t, x) \in Q_{T} g \geq a>0$. We define for a.a. $t \in(0, T)$,

$$
\mathscr{M}_{g(t)} \in \mathscr{L}(X, X), \quad<\mathscr{M}_{g(t)} \Phi, \Psi>_{X}:=\int_{\Omega} g(t, x) \Phi \Psi \mathrm{d} x
$$

With this definition at hand, we easily see that there holds for a.a. $t \in(0, T)$,

$$
\begin{gathered}
\left\|\mathscr{M}_{g(t)}\right\|_{\mathscr{L}(X, X)} \leq \bar{\delta} \int_{\Omega} g(t, x) \mathrm{d} x, \quad<\mathscr{M}_{g(t)} \Phi, \Phi>_{X} \geq a\|\Phi\|_{X}^{2}, \\
\mathscr{M}_{g(t)}^{-1}(t) \in \mathscr{L}(X, X), \quad\left\|\mathscr{M}_{g(t)}^{-1}(t)\right\|_{\mathscr{L}(X, X)} \leq \frac{\bar{\delta}}{a}, \\
\left\|\mathscr{M}_{g_{1}(t)}-\mathscr{M}_{g_{2}(t)}\right\|_{\mathscr{L}(X, X)} \leq \bar{\delta}\left\|g_{1}(t)-g_{2}(t)\right\|_{L^{1}(\Omega)},
\end{gathered}
$$




$$
\left\|\mathscr{M}_{g_{1}(t)}^{-1}-\mathscr{M}_{g_{2}(t)}^{-1}\right\|_{\mathscr{L}(X, X)} \leq \frac{\bar{\delta}}{a^{2}}\left\|g_{1}(t)-g_{2}(t)\right\|_{L^{1}(\Omega)} .
$$

In the above formulas $\bar{\delta}$ is a positive universal number dependent solely of $N$. Moreover, if in addition $g \in C\left([0, T] ; L^{1}(\Omega)\right)$ then

$$
\mathscr{M}_{g(\cdot)}, \mathscr{M}_{g(\cdot)}^{-1} \in C([0, T] ; \mathscr{L}(X, X)) .
$$

Finally, if in addition $\partial_{t} g \in L^{1}\left(Q_{T}\right)$, then

$$
\partial_{t} \mathscr{M}_{g(.)}(t)=\mathscr{M}_{\partial_{t} g(t)} \in \mathscr{L}(X, X), \quad \partial_{t} \mathscr{M}_{g(.)}^{-1}(t)=-\mathscr{M}_{g(t)}^{-1} \mathscr{M}_{\partial t g(t)} \mathscr{M}_{g(t)}^{-1} \in \mathscr{L}(X, X)
$$

for a.a. $t \in(0, T)$.

We shall look for

$$
T^{\prime} \in(0, T] \text { and } \mathbf{u}_{N}=\mathbf{u}_{\infty}+\mathbf{v}_{N}, \mathbf{v}_{N} \in C\left(\left[0, T^{\prime}\right] ; X\right),
$$

satisfying

$$
\begin{gathered}
\int_{\Omega} \partial_{t}\left(\varrho_{N} \mathbf{u}_{N}\right) \cdot \Phi \mathrm{d} x=\int_{\Omega}\left(\operatorname{div} \mathbb{S}\left(\nabla_{x} \mathbf{u}_{N}\right)+\varepsilon \operatorname{div}\left(\left|\nabla_{x}\left(\mathbf{u}_{N}-\mathbf{u}_{\infty}\right)\right|^{2} \nabla_{x}\left(\mathbf{u}_{N}-\mathbf{u}_{\infty}\right)\right)\right. \\
\left.-\nabla_{x} p_{\delta}\left(\varrho_{N}\right)-\operatorname{div}\left(\varrho_{N} \mathbf{u}_{N} \otimes \mathbf{u}_{N}\right)-\varepsilon \nabla_{x} \varrho_{N} \cdot \nabla_{x} \mathbf{u}_{N}\right) \mathrm{d} x,
\end{gathered}
$$

or equivalently, omitting index $N$ at $\mathbf{v}_{N}, \mathbf{u}_{N}$ and $\varrho_{N}$

$$
\begin{gathered}
\int_{\Omega} \varrho \mathbf{v}(t) \cdot \Phi \mathrm{d} x-\int_{\Omega} \varrho_{0} \mathbf{v}_{0} \Phi \mathrm{d} x=\int_{0}^{t} \int_{\Omega}\left(\operatorname{div} \mathbb{S}\left(\nabla_{x} \mathbf{u}\right)+\varepsilon \operatorname{div}\left(\left|\nabla_{x}\left(\mathbf{u}-\mathbf{u}_{\infty}\right)\right|^{2} \nabla_{x}\left(\mathbf{u}-\mathbf{u}_{\infty}\right)\right)\right. \\
\left.-\nabla_{x} p_{\delta}(\varrho)-\operatorname{div}(\varrho \mathbf{u} \otimes \mathbf{u})-\varepsilon \nabla_{x} \varrho \cdot \nabla_{x} \mathbf{u}-\partial_{t} \varrho \mathbf{u}_{\infty}\right) \cdot \Phi \mathrm{d} x \mathrm{~d} t
\end{gathered}
$$

where $\mathbf{v}_{0}=\mathbf{u}_{0}-\mathbf{u}_{\infty}, \varrho(t)=S(\mathbf{u})(t), \Phi \in X, t \in\left(0, T^{\prime}\right)$ with $S$ being defined in item 3. of Lemma 4.3.

The latter equation is equivalent to the integral formulation

$$
\mathbf{v}(t)=\mathscr{T}(\mathbf{v}):=\mathscr{M}_{S(\mathbf{u})(t)}^{-1}\left(P\left(\varrho_{0} \mathbf{v}_{0}\right)\right)+\mathscr{M}_{S(\mathbf{u})(t)}^{-1}\left[\int_{0}^{t}(P \mathcal{N}(S(\mathbf{u})(s), \mathbf{u}(s))) \mathrm{d} s\right],
$$

where

$\mathcal{N}(\varrho, \mathbf{u})=\operatorname{div} \mathbb{S}\left(\nabla_{x} \mathbf{u}\right)+\varepsilon \operatorname{div}\left(\left|\nabla_{x}\left(\mathbf{u}-\mathbf{u}_{\infty}\right)\right|^{2} \nabla_{x}\left(\mathbf{u}-\mathbf{u}_{\infty}\right)\right)-\nabla_{x} p_{\delta}(\varrho)-\operatorname{div}(\varrho \mathbf{u} \otimes \mathbf{u})-\varepsilon \nabla_{x} \varrho \cdot \nabla_{x} \mathbf{u}-\partial_{t} \varrho \mathbf{u}_{\infty}$ and where here and in the sequel, $P$ states for $P_{N}$.

Clearly, $\mathcal{N}(S(\mathbf{u}), \mathbf{u}) \in L^{2}\left(Q_{T^{\prime}}\right)$ due to (4.19), (4.22), (4.31). This implies that 1) the map $t \mapsto$ $\left.P \mathscr{N}(S(\mathbf{u})(t), \mathbf{u}(t)) \in L^{2}(0, T ; X), 2\right)$ the map $t \mapsto \int_{0}^{t}(P \mathcal{N}(S(\mathbf{u})(s), \mathbf{u}(s)) \mathrm{d} s \in C([0, T] ; X), 3)$ operator $\mathscr{T}$ maps $C([0, T] ; X)$ to $C([0, T] ; X), 4)$ we have a bound 


$$
\|P \mathcal{N}(S(\mathbf{u}), \mathbf{u})\|_{X} \leq d_{2}(K, \bar{\varrho}, T), \text { provided } \mathbf{u} \text { verifies }(4.21) .
$$

5) Likewise, using in addition (4.23),

$$
\left\|P \mathscr{N}\left(S\left(\mathbf{u}_{1}\right), \mathbf{u}_{1}\right)-P \mathscr{N}\left(S\left(\mathbf{u}_{2}\right), \mathbf{u}_{2}\right)\right\|_{X} \leq d_{3}(K, \bar{\varrho}, T)\left\|\mathbf{u}_{1}-\mathbf{u}_{2}\right\|_{X} \text {, provided both } \mathbf{u}_{i} \text { verify }(4.21)
$$

Coming back to $\mathscr{T}$, we find with help of (4.27), (4.22), (4.35),

$$
\|\mathscr{T} \mathbf{v}(t)\|_{X} \leq \frac{\bar{\delta}}{\underline{\varrho}} e^{K t}\left(\left\|P \mathbf{v}_{0}\right\|_{X}+d_{2}(K, \bar{\varrho}, T) t\right) \text { provided } \mathbf{u}_{\infty}+\mathbf{v} \text { verifies }(4.21)
$$

and with help of (4.28), (4.22), (4.23) on one hand and (4.27), (3.5) on the other hand

$$
\left\|\mathscr{T} \mathbf{v}_{1}-\mathscr{T} \mathbf{v}_{2}\right\|_{C([0, t] ; X)} \leq t\left(\frac{\Gamma \bar{\delta} d_{2}}{(\underline{\varrho})^{2}}+\frac{\bar{\delta} d_{3}}{\underline{\varrho}}\right) e^{K t}\left\|\mathbf{v}_{1}-\mathbf{v}_{2}\right\|_{C([0, t] ; X)} \text { provided } \mathbf{u}_{\infty}+\mathbf{v}_{i} \text { verifies }(4.21)
$$

Now we take $K>0$ sufficiently large and $T^{\prime}$ sufficiently small, so that

$$
K \frac{\underline{d}}{2} \geq \max \left\{\frac{\bar{\delta}}{\underline{\varrho}} e^{K T^{\prime}}\left(\left\|P \mathbf{v}_{0}\right\|_{X}+d_{2}(K, \bar{\varrho}, T) T^{\prime}\right), \underline{d}\left\|\mathbf{u}_{\infty}\right\|_{W^{1, \infty}(\Omega)}\right\}
$$

and

$$
T^{\prime}\left(\frac{\Gamma \bar{\delta} d_{2}}{(\underline{\varrho})^{2}}+\frac{\bar{\delta} d_{3}}{\underline{\varrho}}\right) e^{K T^{\prime}}<1
$$

With this choice, we easily check employing (4.25) that $\mathbf{u}_{\infty}+\mathbf{v}$ verifies condition (4.21), provided $\|\mathbf{v}\|_{C([0, T] ; X} \leq K \frac{d}{2}$. We have thus showed that $\mathscr{T}$ is a contraction maping from the (closed) ball $B(0 ; K \underline{d} / 2) \subset C\left(\left[0, T^{\prime}\right], X\right)$ into itself. It therefore admits a unique fixed point $\mathbf{v} \in C\left(\left[0, T^{\prime}\right], X\right)$, and $\mathbf{u}=\mathbf{u}_{\infty}+\mathbf{v}$ solves problem (4.33).

Denote now

$$
\mathbb{T}=\left\{T^{\prime} \in(0, T) \mid \text { problem }(4.33) \text { admits a solution } \mathbf{v} \in C\left(\left[0, T^{\prime}\right] ; X\right)\right\}, \quad \text { and set } T_{\max }=\sup \mathbb{T} .
$$

We have already proved that $\mathbb{T}$ is not empty. In what follows we prove that $T_{\max }=T$. In fact, if $T_{\max }<T$, then necessarily

$$
\lim _{T^{\prime} \rightarrow T_{\max }}\|\mathbf{v}\|_{C\left(\left[0, T^{\prime}\right] ; X\right)} \rightarrow \infty .
$$

We shall show that (4.37) cannot happen. To this end we derive in the next section the uniform estimates. 


\subsubsection{Uniform bounds independent of the Galerkin approximation}

We first integrate equation $(4.1)_{\left(\varrho_{N}, \mathbf{u}_{N}\right)}$ in order to obtain the conservation of total mass. Omitting subscript $N$ in order to simplify notatio, we get

$$
\int_{\Omega} \varrho(\tau) \mathrm{d} x+\int_{0}^{\tau} \int_{\Gamma_{\text {out }}} \varrho \mathbf{u}_{B} \cdot \mathbf{n} \mathrm{d} S_{x} \mathrm{~d} t=\int_{\Omega} \varrho_{0} \mathrm{~d} x+\int_{0}^{\tau} \int_{\Gamma_{\text {in }}} \varrho\left|\mathbf{u}_{B} \cdot \mathbf{n}\right| \mathrm{d} S_{x} \mathrm{~d} t
$$

Next, we come back to integral formulation (4.34) and conclude employing (4.27), (4.30) and (4.19) that $\partial_{t} \mathbf{v} \in L^{2}\left(0, T^{\prime} ; X\right)$.

Further, we multiply equation $(4.18)$ by $H_{\delta}^{\prime}(\varrho)$ to deduce

$$
\partial_{t} H_{\delta}(\varrho)+\varepsilon H_{\delta}^{\prime \prime}(\varrho)\left|\nabla_{x} \varrho\right|^{2}-\varepsilon \operatorname{div}\left(H_{\delta}^{\prime}(\varrho) \nabla_{x} \varrho\right)+\operatorname{div}\left(H_{\delta}(\varrho) \mathbf{u}\right)+p_{\delta}(\varrho) \operatorname{div} \mathbf{u}=0 \text { a.e. in } Q_{\tau}, \tau \in\left(0, T^{\prime}\right)
$$

or, after using boundary conditions (4.18),

$\partial_{t} \int_{\Omega} H_{\delta}(\varrho) \mathrm{d} x+\varepsilon \int_{\Omega} H_{\delta}^{\prime \prime}(\varrho)\left|\nabla_{x} \varrho\right|^{2} \mathrm{~d} x+\int_{\partial \Omega}\left(H_{\delta}^{\prime}(\varrho)\left(\varrho_{B}-\varrho\right) v+H_{\delta}(\varrho) \mathbf{u}_{B} \cdot \mathbf{n}\right) \mathrm{d} S_{x}=-\int_{\Omega} p_{\delta}(\varrho) \operatorname{div} \mathbf{u} \mathrm{d} x$ or further, after employing the definition (4.17) of $v$,

$$
\begin{gathered}
\partial_{t} \int_{\Omega} H_{\delta}(\varrho) \mathrm{d} x+\varepsilon \int_{\Omega} H_{\delta}^{\prime \prime}(\varrho)\left|\nabla_{x} \varrho\right|^{2} \mathrm{~d} x+\int_{\Gamma_{\mathrm{in}}}\left(H_{\delta}\left(\varrho_{B}\right)-H_{\delta}^{\prime}(\varrho)\left(\varrho_{B}-\varrho\right)-H_{\delta}(\varrho)\right)|v| \mathrm{d} S_{x}+\int_{\Gamma_{\mathrm{out}}} H_{\delta}(\varrho) \mathbf{u}_{B} \cdot \mathbf{n} \mathrm{d} S_{x} \\
=-\int_{\Omega} p_{\delta}(\varrho) \operatorname{div} \mathbf{u} \mathrm{d} x-\int_{\Gamma_{\mathrm{in}}} H_{\delta}\left(\varrho_{B}\right) \mathbf{u}_{B} \cdot \mathbf{n} \mathrm{d} S_{x}
\end{gathered}
$$

Next, we deduce from $(4.32)$

$$
\begin{gathered}
\int_{0}^{\tau} \int_{\Omega} \partial_{t}(\varrho \mathbf{v}) \cdot \mathbf{v}-\varrho \mathbf{u} \otimes \mathbf{u}: \nabla_{x} \mathbf{v} \mathrm{d} x \mathrm{~d} t+\int_{0}^{\tau} \int_{\Omega} \mathbb{S}\left(\nabla_{x} \mathbf{u}\right): \nabla_{x} \mathbf{v} \mathrm{d} x \\
+\varepsilon \int_{0}^{\tau} \int_{\Omega}\left|\nabla_{x} \mathbf{v}\right|^{4} \mathrm{~d} x \mathrm{~d} t-\int_{0}^{\tau} \int_{\Omega} p_{\delta}(\varrho) \operatorname{div} \mathbf{v} \mathrm{d} x \mathrm{~d} t+\int_{0}^{\tau} \int_{\Omega} \varepsilon \nabla_{x} \varrho \cdot \nabla_{x} \mathbf{u} \cdot \mathbf{v} \mathrm{d} x \mathrm{~d} t=0,
\end{gathered}
$$

where by virtue of (4.18) (after several integrations by parts and recalling that $\mathbf{u}=\mathbf{u}_{\infty}+\mathbf{v}$ ),

$$
\begin{aligned}
\int_{\Omega}\left(\partial_{t}(\varrho \mathbf{u})\right. & \left.\cdot \mathbf{v}-\varrho \mathbf{u} \otimes \mathbf{u}: \nabla_{x} \mathbf{v}\right) \mathrm{d} x=\int_{\Omega}\left(\partial_{t} \varrho \mathbf{v}^{2}+\frac{1}{2} \partial_{t} \varrho \mathbf{v}^{2}+\partial_{t} \varrho \mathbf{u}_{\infty} \cdot \mathbf{v}+\frac{1}{2} \operatorname{div}(\varrho \mathbf{u}) \mathbf{v}^{2}-\varrho \mathbf{u} \cdot \nabla_{x} \mathbf{v} \cdot \mathbf{u}_{\infty}\right) \mathrm{d} x \\
& =\int_{\Omega}\left(\frac{1}{2} \partial_{t}\left(\varrho \mathbf{v}^{2}\right)+\varepsilon \Delta \varrho \mathbf{u}_{\infty} \cdot \mathbf{v}+\frac{\varepsilon}{2} \Delta \varrho \mathbf{v}^{2}-\operatorname{div}(\varrho \mathbf{u}) \mathbf{u}_{\infty} \cdot \mathbf{v}-\varrho \mathbf{u} \cdot \nabla_{x} \mathbf{v} \cdot \mathbf{u}_{\infty}\right) \mathrm{d} x \\
& =\partial_{t} \int_{\Omega} \frac{1}{2} \varrho \mathbf{v}^{2} \mathrm{~d} x+\int_{\Omega}\left(\varrho \mathbf{u} \cdot \nabla_{x} \mathbf{u}_{\infty} \cdot \mathbf{v}-\varepsilon \nabla_{x} \varrho \cdot \nabla_{x} \mathbf{u} \cdot \mathbf{v}-\varepsilon \nabla_{x} \varrho \cdot \nabla_{x} \mathbf{v} \cdot \mathbf{u}_{\infty}\right) \mathrm{d} x
\end{aligned}
$$


Consequently,

$$
\begin{gathered}
\int_{\Omega}\left(\frac{1}{2} \varrho \mathbf{v}^{2}+H_{\delta}(\varrho)\right)(\tau) \mathrm{d} x+\int_{0}^{\tau} \int_{\Gamma_{\text {in }}} E_{\delta}\left(\varrho_{B} \mid \varrho\right)\left|\mathbf{u}_{B} \cdot \mathbf{n}\right| \mathrm{d} S_{x} \mathrm{~d} t+\int_{0}^{\tau} \int_{\Gamma_{\text {out }}} H_{\delta}(\varrho)\left|\mathbf{\mathbf { u } _ { B }} \cdot \mathbf{n}\right| \mathrm{d} S_{x} \mathrm{~d} t \\
+\varepsilon \int_{0}^{\tau} \int_{\Omega}\left|\nabla_{x} \mathbf{v}\right|^{4} \mathrm{~d} x \mathrm{~d} t+\varepsilon \int_{0}^{\tau} \int_{\Omega} H_{\delta}^{\prime \prime}(\varrho)\left|\nabla_{x} \varrho\right|^{2} \mathrm{~d} x \mathrm{~d} t+\int_{0}^{\tau} \int_{\Omega} \mathbb{S}\left(\nabla_{x} \mathbf{v}\right): \nabla_{x} \mathbf{v} \mathrm{d} x \mathrm{~d} t \\
\leq \int_{\Omega}\left(\frac{1}{2} \varrho_{0} \mathbf{v}_{0}^{2}+H_{\delta}\left(\varrho_{0}\right)\right) \mathrm{d} x-\int_{0}^{\tau} \int_{\Gamma_{\mathrm{in}}} H_{\delta}\left(\varrho_{B}\right) \mathbf{u}_{B} \cdot \mathbf{n} \mathrm{d} S_{x} \mathrm{~d} t \\
+\int_{0}^{\tau} \int_{\Omega}\left(-p_{\delta}(\varrho) \operatorname{div} \mathbf{u}_{\infty}-\mathbb{S}\left(\nabla_{x} \mathbf{u}_{\infty}\right): \nabla_{x} \mathbf{v}-\varrho \mathbf{u} \cdot \nabla_{x} \mathbf{u}_{\infty} \cdot \mathbf{v}+\varepsilon \nabla_{x} \varrho \cdot \nabla_{x} \mathbf{v} \cdot \mathbf{u}_{\infty}\right) \mathrm{d} x \mathrm{~d} t
\end{gathered}
$$

where, recall, $H_{\delta}, E_{\delta}$ are defined in (4.6), (4.7), respectively.

Further, we test equation $(4.1)_{\left(\varrho_{N}, \mathbf{u}_{N}\right)}$ by $\varrho_{N}$ in order to get, after several integrations by parts,

$$
\begin{aligned}
& \frac{1}{2} \int_{\Omega} \varrho^{2}(\tau) \mathrm{d} x+\frac{1}{2} \int_{0}^{\tau} \int_{\partial \Omega} \varrho^{2}\left|\mathbf{u}_{B} \cdot \mathbf{n}\right| \mathrm{d} S_{x} \mathrm{~d} t+\varepsilon \int_{0}^{\tau} \int_{\Omega}\left|\nabla_{x} \varrho\right|^{2} \mathrm{~d} x \mathrm{~d} t \\
& =\frac{1}{2} \int_{\Omega} \varrho_{0}^{2} \mathrm{~d} x+\int_{0}^{\tau} \int_{\Gamma_{\mathrm{in}}} \varrho \varrho_{B}\left|\mathbf{u}_{B} \cdot \mathbf{n}\right| \mathrm{d} S_{x} \mathrm{~d} t-\frac{1}{2} \int_{0}^{\tau} \int_{\Omega} \varrho^{2} \operatorname{div} \mathbf{u} \mathrm{d} x \mathrm{~d} t
\end{aligned}
$$

where as in (4.40) we have omitted indexes $N$.

Now, our goal is to derive from (4.40) and (4.41) useful bounds for the sequence $\left(\varrho_{N}, \mathbf{u}_{N}\right)$. The coefficients of derived bounds depend tacitly on the parameters of the problem (as $\gamma, \beta, \mu, \lambda, a, b, \mathrm{~T}$, $\Omega$, functions $p, \mathfrak{p}$ ) and on "data", where

$$
" \text { data" stands for } \int_{\Omega}\left(\frac{1}{2} \varrho_{0} \mathbf{v}_{0}^{2}+H_{\delta}\left(\varrho_{0}\right)\right) \mathrm{d} x,\left\|\mathbf{u}_{\infty}\right\|_{W^{1, \infty}(\Omega)}, \underline{\varrho}_{B}, \bar{\varrho}_{B} \equiv\left\|\varrho_{B}\right\|_{C(\partial \Omega)}, \underline{H}, \underline{\mathfrak{E}} .
$$

In particular, they are always independent $N$. If they depend on $\varepsilon$ or $\delta$ this dependence is also always indicated in their argument as well as the dependence on other quantities (notably $T$ ) if it is necessary for the understanding of proofs. The coefficients may take different values even in the same formulas.

Before attacking estimates we shall list several consequences of structural assumptions (2.2), (4.5) and formulas (2.3), (2.4), (4.6), (4.7) needed for the derivation of those bounds.

1. A brief excursion to (2.3) and (4.6) yields

$$
-\infty<-\frac{\delta}{\beta-1}+\underline{H} \leq \underline{H}_{\delta} .
$$

2. We shall now investigate the lower bound of $E_{\delta}\left(\varrho_{B} \mid \varrho\right)$. First, due to convexity of $\mathscr{H}$, we have $\mathscr{E}\left(\varrho_{B} \mid \varrho\right) \geq 0$. Second, we verify by direct calculation that

$$
E^{(\beta)}(r \mid \varrho) \geq \frac{1}{2} \varrho^{\beta}-\frac{2^{\beta-1}}{\beta-1} r^{\beta},
$$


where $E^{(\beta)}$ is defined in (4.7). Finally, using namely conditions $\mathfrak{p}^{\prime}(0)=0$ (cf. last line in formula $(2.2))$, we get

$$
\underline{\mathfrak{E}}:=\inf _{\varrho>0 ; \underline{\varrho}_{B}<r<\bar{\varrho}_{B}} \mathfrak{E}(r \mid \varrho)>-\infty
$$

Putting together these three observations we infer (see (4.6-4.7) for the notation),

$$
E_{\delta}\left(\varrho_{B} \mid \varrho\right) \geq \frac{\delta}{2} \varrho^{\beta}+\underline{\mathfrak{E}}-\delta \frac{2^{\beta-1}}{\beta-1} \bar{\varrho}_{B}^{\beta}
$$

3. Recalling again definition $(4.7)$ of $E_{\delta}, \mathscr{E}_{\delta}$ we find identity

$$
H_{\delta}(\varrho)=\mathscr{E}_{\delta}(\varrho \mid \underline{\underline{\varrho}})+D_{\delta}(\varrho, \underline{\underline{\varrho}}),
$$

where

$$
D_{\delta}(\varrho, \bar{\varrho})=\left[\mathscr{H}_{\delta}^{\prime}(\underline{\underline{\varrho}})(\varrho-\underline{\bar{\varrho}})+\mathscr{H}_{\delta}(\underline{\bar{\varrho}})+\mathfrak{H}(\varrho)\right], \quad \bar{\varrho}=\frac{1}{|\Omega|} \int_{\Omega} \varrho_{0} \mathrm{~d} x
$$

Thanks to (4.38),

$$
\sup _{t \in(0, T)} \int_{\Omega}|D(\varrho, \underline{\underline{Q}})| \mathrm{d} x \leq c(\text { data }),
$$

where we have also employed regularity of $\mathfrak{p}$ near zero to show that the map $\varrho \mapsto \mathfrak{H}(\varrho)$ is bounded near 0 .

4. We have $\mathscr{E}_{\delta}(\varrho \mid r)=\mathscr{E}(\varrho \mid r)+\delta E^{(\beta)}(\varrho \mid r)$, where, due to convexity of $\mathscr{H}$ and $H^{(\beta)}$ on $(0, \infty)$, $\mathscr{E}_{\delta}$ enjoys the following coercivity property: There is $c=c(\underline{\underline{Q}})>0$, such that

$$
\begin{gathered}
\mathscr{E}_{\delta}(\varrho \mid \underline{\underline{\varrho}}) \geq c\left[\delta \left(\varrho^{\beta} 1_{\mathcal{O}_{\text {res }}}(\varrho)+1_{\mathcal{O}_{\text {res }}}(\varrho)\right.\right. \\
\left.\left.+1_{\mathcal{O}_{\text {ess }}}(\varrho)(\varrho-\underline{\underline{\varrho}})^{2}\right)+\left(\varrho^{\gamma} 1_{\mathcal{O}_{\text {res }}}(\varrho)+1_{\mathcal{O}_{\text {res }}}(\varrho)+1_{\mathcal{O}_{\text {ess }}}(\varrho)(\varrho-\underline{\underline{\varrho}})^{2}\right)\right]
\end{gathered}
$$

for all $\varrho \geq 0$, where $\mathcal{O}_{\text {ess }}=\left(\frac{1}{2} \underline{\underline{\underline{\varrho}}}, 2 \underline{\underline{\varrho}}\right)$ while $\mathcal{O}_{\text {res }}=[0, \infty) \backslash \mathcal{O}_{\text {ess }}$, and where we have used the growth condition for $p$ from $(2.2)$.

5. We deduce from the Korn and Poincaré inequalities,

$$
\|\mathbf{v}\|_{W^{1,2}(\Omega)}^{2} \leq c\left\|\mathbb{S}\left(\nabla_{x} \mathbf{v}\right): \nabla_{x} \mathbf{v}\right\|_{L^{1}(\Omega)}
$$

6. There holds $H_{\delta}=\mathscr{H}+\delta H^{(\beta)}+\mathfrak{H}$, where

$$
\begin{gathered}
\mathscr{H}^{\prime \prime} \geq 0,\left[H^{(\beta)}\right]^{\prime \prime}(\varrho)=\beta \varrho^{\beta-2} \\
\int_{0}^{T} \int_{\Omega}\left|\mathfrak{H}^{\prime \prime}(\varrho)\right|\left|\nabla_{x} \varrho\right|^{2} \mathrm{~d} x \mathrm{~d} t \leq \sup _{\varrho>0}\left|\frac{\mathfrak{p}^{\prime}(\varrho)}{\varrho}\right| \int_{0}^{T} \int_{\Omega}\left|\nabla_{x} \varrho\right|^{2} \mathrm{~d} x \mathrm{~d} t,
\end{gathered}
$$

where $\sup _{\varrho>0}\left|\frac{\mathfrak{p}^{\prime}(\varrho)}{\varrho}\right|<\infty$ namely thanks to assumption $\mathfrak{p}^{\prime}(0)=0$ (cf. again last line in formula $(2.2))$. 
7. The absolute value of the right hand side of inequality (4.40) is bounded by

$$
\begin{gathered}
\frac{1+\alpha}{\alpha} c(\text { data, } \delta) \int_{0}^{\tau} \int_{\Omega}\left(\mathscr{E}_{\delta}(\varrho \mid \underline{\underline{\varrho}})+\frac{1}{2} \varrho \mathbf{v}^{2}\right) \mathrm{d} x \mathrm{~d} t+\alpha \varepsilon\left\|\nabla_{x} \varrho\right\|_{L^{2}\left(Q_{\tau}\right)}^{2} \\
+\left(\alpha+\varepsilon \frac{c(\text { data }, T)}{\alpha}\right)\left\|\nabla_{x} \mathbf{v}\right\|_{L^{2}\left(Q_{\tau}\right)}^{2}+\frac{c(\text { data })}{\alpha},
\end{gathered}
$$

with arbitrary $\alpha>0$, where we have used several times the Hölder and Young inequalities, and coercivity $(4.46)$ of $\mathscr{E}_{\delta}(\varrho \mid \underline{\underline{ }})$.

8. By the same token, the absolute value of the right hand side of equality (4.41) is bounded by

$$
\begin{aligned}
& \frac{1}{\delta} \frac{1+\alpha}{\alpha} c(\text { data, } T)+c \frac{1+\alpha}{\alpha} \frac{1}{\delta} \int_{0}^{\tau} \int_{\Omega} \mathscr{E}_{\delta}(\varrho \mid \underline{\underline{\varrho}}) \mathrm{d} x \mathrm{~d} t \\
& \quad+\alpha \delta \int_{0}^{\tau} \int_{\Gamma_{\mathrm{in}}} \varrho^{\beta}\left|\mathbf{u}_{B} \cdot \mathbf{n}\right| \mathrm{d} S_{x} \mathrm{~d} t+\alpha\left\|\nabla_{x} \mathbf{v}\right\|_{L^{2}\left(Q_{\tau}\right)}^{2}
\end{aligned}
$$

with arbitrary $\alpha>0$.

Next, we multiply equation (4.41) by a positive number $\Sigma$ and add it to inequality (4.40). With notably (4.42-4.43) at hand, we deduce from this operation the following inequality which will be our departure point:

$$
\begin{gathered}
\int_{\Omega}\left(\frac{1}{2} \varrho \mathbf{v}^{2}+H_{\delta}(\varrho)+\frac{\Sigma}{2} \varrho^{2}\right)(\tau) \mathrm{d} x \\
+\delta \frac{\beta-1}{2} \int_{0}^{\tau} \int_{\Gamma_{\mathrm{in}}} \varrho^{\beta}\left|\mathbf{u}_{B} \cdot \mathbf{n}\right| \mathrm{d} S_{x} \mathrm{~d} t+\delta \frac{1}{\beta-1} \int_{0}^{\tau} \int_{\Gamma_{\text {out }}} \varrho^{\beta}\left|\mathbf{u}_{B} \cdot \mathbf{n}\right| \mathrm{d} S_{x} \mathrm{~d} t+\frac{\Sigma}{2} \int_{0}^{\tau} \int_{\partial \Omega} \varrho^{2}\left|\mathbf{u}_{B} \cdot \mathbf{n}\right| \mathrm{d} S_{x} \mathrm{~d} t \\
+\int_{0}^{\tau} \int_{\Omega}\left(\mathbb{S}\left(\nabla_{x} \mathbf{v}\right): \nabla_{x} \mathbf{v}+\varepsilon H_{\delta}^{\prime \prime}(\varrho)\left|\nabla_{x} \varrho\right|^{2}+\varepsilon \Sigma\left|\nabla_{x} \varrho_{\varepsilon}\right|^{2}+\varepsilon\left|\nabla_{x} \mathbf{v}\right|^{4}\right) \mathrm{d} x \mathrm{~d} t \\
\leq \int_{\Omega}\left(\frac{1}{2} \varrho_{0} \mathbf{v}_{0}^{2}+H_{\delta}\left(\varrho_{0}\right)+\frac{\Sigma}{2} \varrho_{0}^{2}\right) \mathrm{d} x \\
-(\underline{E}-\delta B) \int_{0}^{\tau} \int_{\Gamma_{\mathrm{in}}}\left|\mathbf{u}_{B} \cdot \mathbf{n}\right| \mathrm{d} S_{x} \mathrm{~d} t-(\underline{H}-\delta A) \int_{0}^{\tau} \int_{\Gamma_{\mathrm{out}}}\left|\mathbf{u}_{B} \cdot \mathbf{n}\right| \mathrm{d} S_{x} \mathrm{~d} t \\
-\int_{0}^{\tau} \int_{\Gamma_{\mathrm{in}}} H_{\delta}\left(\varrho_{B}\right) \mathbf{u}_{B} \cdot \mathbf{n} \mathrm{d} S_{x} \mathrm{~d} t+\Sigma \int_{0}^{\tau} \int_{\Gamma_{\mathrm{in}}} \varrho \varrho_{B}\left|\mathbf{u}_{B} \cdot \mathbf{n}\right| \mathrm{d} S_{x} \mathrm{~d} t \\
-\int_{0}^{\tau} \int_{\Omega} p_{\delta}(\varrho) \mathrm{div} \mathbf{u}_{\infty} \mathrm{d} x \mathrm{~d} t-\int_{0}^{\tau} \int_{\Omega} \varrho \mathbf{u} \cdot \nabla_{x} \mathbf{u}_{\infty} \cdot \mathbf{v} \mathrm{d} x \mathrm{~d} t \\
+\varepsilon \int_{0}^{\tau} \int_{\Omega} \nabla_{x} \varrho_{\varepsilon} \cdot \nabla_{x} \mathbf{v} \cdot \mathbf{u}_{\infty} \mathrm{d} x \mathrm{~d} t-\int_{0}^{\tau} \int_{\Omega} \mathbb{S}\left(\nabla_{x} \mathbf{u}_{\infty}\right): \nabla_{x} \mathbf{v} \mathrm{d} x \mathrm{~d} t-\frac{\Sigma}{2} \int_{0}^{\tau} \int_{\Omega} \varrho^{2} \mathrm{div} \mathbf{u} \mathrm{d} x \mathrm{~d} t
\end{gathered}
$$

where numbers $A, B$ are define in (4.11). 
Now we take in $(4.52)$

$$
\Sigma=2 \sup _{\varrho>0}\left|\frac{\mathfrak{p}^{\prime}(\varrho)}{\varrho}\right|
$$

and use estimates (4.44-4.45) when dealing with $\int_{\Omega} H_{\delta}(\varrho)(\tau) \mathrm{d} x,(4.47)$ when dealing with $\int_{0}^{\tau} \int_{\Omega} \mathbb{S}\left(\nabla_{x} \mathbf{v}\right)$ : $\nabla_{x} \mathbf{v} \mathrm{d} x \mathrm{~d} t,(4.48-4.49)$ when treating $\varepsilon \int_{0}^{\tau} \int_{\Omega} H_{\delta}^{\prime \prime}(\varrho)\left|\nabla_{x} \varrho\right|^{2} \mathrm{~d} x \mathrm{~d} t$, and (4.50-4.51) to treat the right hand side (while taking first $\alpha>0$ sufficiently small and then $\varepsilon>0$ also sufficiently small in order to let the terms $\alpha \varepsilon\left\|\nabla_{x} \varrho\right\|_{L^{2}\left(Q_{\tau}\right)},\left(\alpha+\varepsilon \frac{c}{\alpha}\right)\left\|\nabla_{x} \mathbf{v}\right\|_{L^{2}\left(Q_{\tau}\right)}^{2}$ and $\alpha \delta \int_{0}^{\tau} \int_{\Gamma_{\mathrm{in}}} \varrho^{\beta}|\mathbf{u} \cdot \mathbf{n}| \mathrm{d} S_{x}$ "absorb" in the left hand side) with the goal to obtain with help of the Gronwall inequality,

$$
\begin{gathered}
\sup _{t \in\left(0, T^{\prime}\right)} \int_{\Omega} \varrho \mathbf{v}^{2}(t) \mathrm{d} x \leq \bar{K}(\text { data }, T, \delta) \\
\|\mathbf{v}\|_{L^{2}\left(0, T^{\prime} ; W^{1,2}(\Omega)\right)} \leq \bar{K}(\text { data }, T, \delta), \\
\sup _{t \in\left(0, T^{\prime}\right)} \int_{\Omega} \mathscr{E}_{\delta}(\varrho \mid \underline{\varrho}) \mid(t) \mathrm{d} x \leq L(\text { data }, T, \delta), \\
\varepsilon \int_{0}^{T^{\prime}} \int_{\Omega} \mathscr{H}_{\delta}^{\prime \prime}(\varrho)\left|\nabla_{x} \varrho\right|^{2} \mathrm{~d} x \leq L(\text { data }, T, \delta), \\
\varepsilon \int_{0}^{T^{\prime}} \int_{\Omega}\left|\nabla_{x} \varrho\right|^{2} \mathrm{~d} x \leq L(\text { data }, T, \delta), \\
\int_{0}^{T^{\prime}} \int_{\partial \Omega} \varrho^{\beta}\left|\mathbf{u}_{B} \cdot \mathbf{n}\right| \mathrm{d} S_{x} \mathrm{~d} t \leq L(\text { data }, T, \delta) . \\
\varepsilon\|\mathbf{v}\|_{L^{4}\left(0, T^{\prime} ; W^{1,4}(\Omega)\right)}^{4} \leq L(\text { data }, T, \delta) .
\end{gathered}
$$

At this immediate stage, we shall use the first two estimates. Employing (4.25), namely $\|\mathbf{v}\|_{W^{1, \infty}(\Omega)} \leq$ $\bar{c}\|\mathbf{v}\|_{W^{1,2}(\Omega)}, \mathbf{v} \in X$, and $(4.22)$, we get

$$
\varrho \geq \underline{\varrho} \exp \left(-\int_{0}^{T^{\prime}}\left(\left\|\mathbf{u}_{\infty}(s)\right\|_{W^{1, \infty}(\Omega)}+\bar{c}\|\mathbf{v}(s)\|_{W^{1,2}(\Omega)}\right) \mathrm{d} s\right) \geq K_{1}(\underline{\varrho}, T, \text { data }) .
$$

Coming back to (4.53), and using $\|\mathbf{v}\|_{L^{2}(\Omega)} \geq \underline{d}\|\mathbf{v}\|_{W^{1, \infty}(\Omega)}$, we finally obtain

$$
\|\mathbf{v}\|_{C\left(\left[0, T^{\prime}\right] ; W^{1, \infty}(\Omega)\right)} \leq \frac{1}{\underline{d}} \frac{\bar{K}}{K_{1}} \text { for any } T^{\prime}<T_{\max } .
$$

This contradicts (4.37). We have thus proved that $T_{\max }=T$. 


\subsubsection{From Galerkin approximation to solutions of approximate problem (4.1-4.4)}

Recalling structural assumptions (2.2) for $p$, definitions (2.3) of $H$ and (4.6) of $p_{\delta}, H_{\delta}$ (notably the coercivity relations $(4.46),(4.48)$, we deduce from (4.53-4.59) the following bounds for the sequence $\left(\varrho_{N}=S\left(\mathbf{u}_{N}\right), \mathbf{u}_{N}=\mathbf{u}_{\infty}+\mathbf{v}_{N}\right)$ of Galerkin solutions to the problem (4.33):

$$
\begin{gathered}
\left\|\varrho_{N}\left|\mathbf{u}_{N}\right|^{2}\right\|_{L^{\infty}\left(I, L^{1}(\Omega)\right)} \leq L(\text { data }, \delta), \\
\left\|\mathbf{u}_{N}\right\|_{L^{2}\left(I, W^{1,2}(\Omega)\right)} \leq L(\text { data }, \delta), \\
\left\|\varrho_{N}\right\|_{L^{\infty}\left(I, L^{\beta}(\Omega)\right)} \leq L(\text { data }, \delta), \\
\varepsilon\left\|\nabla \varrho_{N}\right\|_{L^{2}\left(Q_{T}\right)}^{2}+\varepsilon\left\|\nabla\left(\varrho_{N}^{\beta / 2}\right)\right\|_{L^{2}\left(Q_{T}\right)}^{2} \leq L(\text { data }, \delta), \\
\left\|\varrho\left|\mathbf{u}_{B} \cdot \mathbf{n}\right|^{1 / \beta}\right\|_{L^{\beta}((0, T) \times \partial \Omega)} \leq L(\text { data }, \delta), \\
\varepsilon\left\|\mathbf{u}_{N}-\mathbf{u}_{\infty}\right\|_{L^{4}\left(0, T ; W^{1,4}(\Omega)\right)}^{4} \leq L(\text { data }, \delta) .
\end{gathered}
$$

From these bounds we find by Hölder inequalities, Sobolev embeddings and interpolation

$$
\begin{gathered}
\left\|\varrho_{N} \mathbf{u}_{N}\right\|_{L^{\infty}\left(I, L^{\frac{2 \beta}{\beta+1}}(\Omega)\right)}+\left\|\varrho_{N} \mathbf{u}_{N}\right\|_{L^{2}\left(I, L^{\frac{6 \beta}{\beta+6}}(\Omega)\right)} \leq L(\text { data }, \delta), \\
\left\|\varrho_{N}\left|\mathbf{u}_{N}\right|^{2}\right\|_{L^{2}\left(I, L^{\frac{6 \beta}{4 \beta+3}}(\Omega)\right)} \leq L(\text { data }, \delta), \\
\left\|\varrho_{N}\right\|_{L^{\frac{5}{3} \beta}\left(Q_{T}\right)} \leq L(\text { data }, \delta, \varepsilon), \\
\left\|\operatorname{div}\left(\varrho_{N} \mathbf{u}_{N}\right)\right\|_{L^{4 / 3}\left(Q_{T}\right)} \leq L(\text { data, } \delta, \varepsilon) .
\end{gathered}
$$

Now we return to $(4.18)$-with $\left(\varrho_{N}, \mathbf{u}_{N}\right)$ - and consider it as parabolic problem with operatot $\partial_{t} \varrho-\varepsilon \Delta \varrho$ in $(0, T) \times \Omega$ with right hand side $-\operatorname{div}\left(\varrho_{N} \mathbf{u}_{N}\right)$, and boundary operator $-\varepsilon \mathbf{n} \cdot \nabla_{x} \varrho+v \varrho$ in $(0, T) \times \partial \Omega$ with right hand side $\varrho_{B} v$. The maximal parabolic regularity theory, as e.g. [4, Theorem 2.1], yields that

$$
\left\|\partial_{t} \varrho_{N}\right\|_{L^{4 / 3}\left(Q_{T}\right)}+\left\|\varrho_{N}\right\|_{L^{4 / 3}\left(0, T ; W^{2,4 / 3}(\Omega)\right)} \leq L(\text { data, } \delta, \varepsilon) .
$$

The above bounds imply, via several classical convergence theorems, existence of a chosen subsequence (not relabeled) whose limits and way of convergence will be specified in the following text.

We deduce from (4.70),

$$
\varrho_{N} \rightarrow \varrho \text { in } L^{4 / 3}\left(0, T ; W^{2,4 / 3}(\Omega)\right) \text { and in } L^{2}\left(0, T ; W^{1,2}(\Omega)\right), \quad \partial_{t} \varrho_{N} \rightarrow \partial_{t} \varrho \text { in } L^{4 / 3}\left(Q_{T}\right)
$$

and also in addition with help of (4.62-4.63) by Lions-Aubin Lemma, $\varrho_{N} \rightarrow \varrho$ in $L^{2}\left(Q_{T}\right), \nabla_{x} \varrho_{N} \rightarrow \nabla_{x} \varrho$ in $L^{4 / 3}\left(Q_{T}\right)$; whence, in particular,

$$
\begin{gathered}
\varrho_{N} \rightarrow \varrho \text { a.e. in } Q_{T} \text { and in } L^{p}\left(Q_{T}\right), 1 \leq p<\frac{5}{3} \beta, \\
\nabla_{x} \varrho_{N} \rightarrow \nabla_{x} \varrho \text { a.e. in } Q_{T} \text { and in } L^{p}\left(Q_{T}\right), 1 \leq p<2,
\end{gathered}
$$


where we have used (4.68) and (4.63). Consequently, in particular

$$
p_{\delta}\left(\varrho_{N}\right), H_{\delta}\left(\varrho_{N}\right) \rightarrow p_{\delta}(\varrho), H_{\delta}(\varrho) \text { in } L^{p / \beta}\left(Q_{T}\right), \beta<p<\frac{5}{3} \beta .
$$

Further, due to (4.63), trace theorem and (4.64)

$$
\varrho_{N} \rightarrow \varrho \text { in } L^{2}((0, T) \times \partial \Omega), \varrho_{N}\left|\mathbf{u}_{B} \cdot \mathbf{n}\right|^{1 / \beta} \rightarrow \varrho\left|\mathbf{u}_{B} \cdot \mathbf{n}\right|^{1 / \beta} \text { in } L^{\beta}((0, T) \times \partial \Omega) .
$$

Next, we derive from (4.18) written with $\left(\varrho_{N}, \mathbf{u}_{N}\right)$ that the sequences of functions $t \mapsto \int_{\Omega} \varrho_{N}(t) \varphi \mathrm{d} x$ are for any $\varphi \in C_{c}^{1}(\Omega)$ uniformly bounded and equi-continuous in $C[0, T]$; whence the Arzela-Ascoli theorem in combination with the separability of $L^{\beta^{\prime}}(\Omega)$ furnishes,

$$
\varrho_{N} \rightarrow \varrho \text { in } C_{\text {weak }}\left([0, T], L^{\beta}(\Omega)\right) .
$$

Estimate (4.65) yields

$$
\mathbf{u}_{N} \rightarrow \mathbf{u} \text { (weakly) in } L^{4}\left(0, T ; W^{1,4}(\Omega)\right),
$$

and in combination with $(4.72)$

$$
\varrho_{N} \mathbf{u}_{N} \rightarrow \varrho \mathbf{u} \text { e.g. in } L^{2}\left(0, T ; L^{\frac{6 \beta}{\beta+6}}(\Omega)\right)
$$

and finally, together with (4.69),

$$
\left.\operatorname{div}\left(\varrho_{N} \mathbf{u}_{N}\right) \rightarrow \operatorname{div}(\varrho \mathbf{u}) \text { in } L^{4 / 3} Q_{T}\right) .
$$

Estimate (4.65) furnishes further

$$
\varepsilon\left|\nabla_{x}\left(\mathbf{u}_{N}-\mathbf{u}_{\infty}\right)\right|^{2} \nabla_{x}\left(\mathbf{u}_{N}-\mathbf{u}_{\infty}\right) \rightarrow \mathbf{Z} \equiv \mathbf{Z}_{\varepsilon} \text { weakly in } L^{4 / 3}\left(Q_{T} ; R^{9}\right),
$$

where

$$
\left\|\mathbf{Z}_{\varepsilon}\right\|_{L^{4 / 3}\left(Q_{T}\right)} \rightarrow 0 \text { as } \varepsilon \rightarrow 0 .
$$

Second convergence in (4.73) and (4.77) yield

$$
\nabla_{x} \varrho_{N} \cdot \nabla_{x} \mathbf{u}_{N} \rightarrow \nabla_{x} \varrho \cdot \nabla_{x} \mathbf{u} \text { in } L^{4 / 3}\left(Q_{T}\right) .
$$

Returning with estimates (4.60-4.67) and with (4.81) to (4.33), we infer that the sequences of functions $t \mapsto \int \varrho_{N} \mathbf{u}_{N}(t) \Phi_{i}$ are for any $\Phi_{i} \in \mathscr{B}$ uniformly bounded and equi-continuous in $C[0, T]$. We may thus combine Arzela-Ascoli theorem with the fact that the linear hull of $\mathscr{B}$ is dense in $L^{\frac{2 \beta}{\beta-1}}(\Omega)$ to deduce that

$$
\varrho_{N} \mathbf{u}_{N} \rightarrow \varrho \mathbf{u} \text { in } C_{\text {weak }}\left([0, T] ; L^{\frac{2 \beta}{\beta+1}}(\Omega)\right),
$$

where we have used the second convergence in (4.77) in order to identify the limit. Seeing the compact imbedding $L^{\frac{2 \beta}{\beta+1}}(\Omega) \hookrightarrow \hookrightarrow W^{-1,2}(\Omega)$, we deduce $\varrho_{N} \mathbf{u}_{N}(t) \rightarrow \varrho \mathbf{u}(t)$ (strongly) in $W^{-1,2}(\Omega)$ for all $t \in[0, T]$. 
This implies, in particular, $\varrho_{N} \mathbf{u}_{N} \rightarrow \varrho \mathbf{u}$ in $L^{2}\left(0, T ; W^{-1,2}(\Omega)\right)$. Combining weak convergence (4.77) with just obtained strong convergence of $\varrho_{N} \mathbf{u}_{N}$, and with estimate (4.67) we get

$$
\varrho_{N} \mathbf{u}_{N} \otimes \mathbf{u}_{N} \rightarrow \varrho \mathbf{u} \otimes \mathbf{u} \text { in } L^{2}\left(I, L^{\frac{6 \beta}{4 \beta+3}}(\Omega)\right)
$$

Relations (4.72-4.83) guarantee the belonging of $(\varrho, \mathbf{u})$ to class $(4.8)$ while $(4.63),(4.68)$ and (4.70) guarantee additional regularity (4.15). Relation (4.71) guarantees that equation (4.9) is satisfied in the strong sense (4.1). Equation (4.1) $\varrho_{\varepsilon}, \mathbf{u}_{\varepsilon}$ tested by $\varrho_{e}$ yields inequality (4.41) by the same manipulations as presented during the derivation of inequality (4.41). Equation (4.16) is obtained by multiplying (4.1) by $b^{\prime}(\varrho)$. The limits (4.72-4.78), (4.81-4.83) employed in (4.32) lead to equation (4.10). It remains to pass to the limit from the inequality $(4.52)_{\left(\varrho_{N}, \mathbf{u}_{N}\right)}$ to inequality (4.11). To this end, we use at the left hand side the lower weak semi-continuity of norms and convex functionals. The right hand side converges to its due limit (the same expression with $(\varrho, \mathbf{v}))$ due to $(4.72),(4.77),(4.81),(4.83)$ and $(4.75)$. We postpone the details of the limit passage in the energy inequality to the next Section, where similar reasoning will be employed. We have thus established Lemma 4.1.

\section{Limit $\varepsilon \rightarrow 0$}

The aim in this section is to pass to the limit in the weak formulation (4.9-4.11) of the problem (4.1$\left.4^{4.4}\right)_{\left(\varrho_{\varepsilon}, \mathbf{u}_{\varepsilon}\right)}$ in order to recover the weak formulation of problem (1.1-1.6) written with $p_{\delta}, H_{\delta}$ instead of $p, H$ (cf. (4.8-4.11)). We expect that there is a (weak) limit $(\varrho, \mathbf{u})$ of a conveniently chosen subsequence $\left(\varrho_{\varepsilon}, \mathbf{u}_{\varepsilon}\right)$, that represents a weak solution of problem $(1.1-1.6)_{\left(p=p_{\delta}, H=H_{\delta}\right)}(\mathrm{cf} .(2.5-2.9))$. More exactly, we want to prove the following lemma:

Lemma 5.1. Under assumptions of Lemma 4.1, there exists a subsequence $\left(\varrho_{\varepsilon}, \mathbf{u}_{\varepsilon}\right)$ (not relabeled) and a couple $(\varrho, \mathbf{u})$ such that

$$
\begin{gathered}
\varrho_{\varepsilon} \rightarrow_{*} \varrho(\text { weakly }-*) \text { in } L^{\infty}\left(0, T ; L^{\beta}(\Omega)\right), \mathbf{u}_{\varepsilon} \rightarrow \mathbf{u} \in L^{2}\left(0, T ; W^{1,2}\left(\Omega ; R^{3}\right)\right) \\
0 \leq \varrho \text { a.a. in }(0, T) \times \Omega,\left.\quad \mathbf{u}\right|_{(0, T) \times \partial \Omega}=\mathbf{u}_{B}
\end{gathered}
$$

satisfying:

1. Function $\varrho \in C_{\text {weak }}\left([0, T], L^{\beta}(\Omega)\right)$ and the integral identity

$$
\begin{gathered}
\int_{\Omega} \varrho(\tau, \cdot) \varphi(\tau, \cdot) \mathrm{d} x-\int_{\Omega} \varrho_{0}(\cdot) \varphi(0, \cdot) \mathrm{d} x \\
=\int_{0}^{\tau} \int_{\Omega}\left(\varrho \partial_{t} \varphi+\varrho \mathbf{u} \cdot \nabla_{x} \varphi\right) \mathrm{d} x \mathrm{~d} t-\int_{0}^{\tau} \int_{\Gamma_{\mathrm{in}}} \varrho_{B} \mathbf{u}_{B} \cdot \mathbf{n} \varphi \mathrm{d} S_{x} \mathrm{~d} t
\end{gathered}
$$

holds for any $\tau \in[0, T]$ and $\varphi \in C_{c}^{1}\left([0, T] \times\left(\Omega \cup \Gamma_{\text {in }}\right)\right)$; 
2. The renormalized continuity equations holds:

$$
\begin{gathered}
\int_{\Omega} b(\varrho) \varphi(\tau) \mathrm{d} x-\int_{\Omega} b\left(\varrho_{0}\right) \varphi(0) \mathrm{d} x= \\
\int_{0}^{\tau} \int_{\Omega}\left(b(\varrho) \partial_{t} \varphi+b(\varrho) \mathbf{u} \cdot \nabla_{x} \varphi-\varphi\left(b^{\prime}(\varrho) \varrho-b(\varrho)\right) \operatorname{div}_{x} \mathbf{u}\right) \mathrm{d} x \mathrm{~d} t-\int_{0}^{\tau} \int_{\Gamma_{\mathrm{in}}} b\left(\varrho_{B}\right) \mathbf{u}_{B} \cdot \mathbf{n} \varphi \mathrm{d} S_{x} \mathrm{~d} t
\end{gathered}
$$

for any $\varphi \in C_{c}^{1}\left([0, T] \times\left(\Omega \cup \Gamma_{\text {in }}\right)\right)$, and any continuously differentiable $b$ with $b^{\prime}$ having a compact support in $[0, \infty)$.

3. Function $\varrho \mathbf{u} \in C_{\text {weak }}\left([0, T], L^{\frac{2 \beta}{\beta+1}}\left(\Omega ; R^{3}\right)\right)$, and the integral identity

$$
\begin{gathered}
\int_{\Omega} \varrho \mathbf{u}(\tau, \cdot) \cdot \boldsymbol{\varphi}(\tau, \cdot) \mathrm{d} x-\int_{\Omega} \varrho_{0} \mathbf{u}_{0}(\cdot) \boldsymbol{\varphi}(0, \cdot) \mathrm{d} x \\
=\int_{0}^{\tau} \int_{\Omega}\left(\varrho \mathbf{u} \cdot \partial_{t} \boldsymbol{\varphi}+\varrho \mathbf{u} \otimes \mathbf{u}: \nabla_{x} \boldsymbol{\varphi}+p_{\delta}(\varrho) \operatorname{div}_{x} \boldsymbol{\varphi}-\mathbb{S}\left(\nabla_{x} \mathbf{u}\right): \nabla_{x} \boldsymbol{\varphi}\right) \mathrm{d} x \mathrm{~d} t
\end{gathered}
$$

holds for any $\tau \in[0, T]$ and any $\varphi \in C_{c}^{1}\left([0, T] \times \Omega ; R^{3}\right)$.

4. The energy inequality

$$
\begin{gathered}
\int_{\Omega}\left(\frac{1}{2} \varrho\left|\mathbf{u}-\mathbf{u}_{\infty}\right|^{2}+H_{\delta}(\varrho)\right)(\tau) \mathrm{d} x+\int_{0}^{\tau} \int_{\Omega} \mathbb{S}\left(\nabla_{x}\left(\mathbf{u}-\mathbf{u}_{\infty}\right)\right): \nabla_{x}\left(\mathbf{u}-\mathbf{u}_{\infty}\right) \mathrm{d} x \mathrm{~d} t \\
\leq \int_{\Omega}\left(\frac{1}{2} \varrho_{0}\left|\mathbf{u}_{0}-\mathbf{u}_{\infty}\right|^{2}+H\left(\varrho_{0}\right)\right) \mathrm{d} x-\int_{0}^{\tau} \int_{\Gamma_{\mathrm{in}}} H_{\delta}\left(\varrho_{B}\right) \mathbf{u}_{B} \cdot \mathbf{n} \mathrm{d} S_{x} \mathrm{~d} t \\
-(\underline{\underline{E}}-\delta B) \int_{0}^{\tau} \int_{\Gamma_{\mathrm{in}}}\left|\mathbf{u}_{B} \cdot \mathbf{n}\right| \mathrm{d} S_{x} \mathrm{~d} t-(\underline{H}-\delta A) \int_{0}^{\tau} \int_{\Gamma_{\text {out }}}\left|\mathbf{u}_{B} \cdot \mathbf{n}\right| \mathrm{d} S_{x} \mathrm{~d} t \\
\quad-\int_{0}^{\tau} \int_{\Omega} p_{\delta}(\varrho) \operatorname{div} \mathbf{u}_{\infty} \mathrm{d} x \mathrm{~d} t-\int_{0}^{\tau} \int_{\Omega} \varrho \mathbf{u} \cdot \nabla_{x} \mathbf{u}_{\infty} \cdot\left(\mathbf{u}-\mathbf{u}_{\infty}\right) \mathrm{d} x \mathrm{~d} t \\
-\int_{0}^{\tau} \int_{\Omega} \mathbb{S}\left(\nabla_{x} \mathbf{u}_{\infty}\right): \nabla_{x}\left(\mathbf{u}-\mathbf{u}_{\infty}\right) \mathrm{d} x \mathrm{~d} t
\end{gathered}
$$

holds for a.a. $\tau \in(0, T)$. Numbers $A, B$ are defined in (4.11) and $\underline{H}$, $\underline{E}$ in (2.10) and (4.12), respectively. Vector field $\mathbf{u}_{\infty}$ is a given continuous extension of $\mathbf{u}_{B}$ in class (2.11).

The remaining part of Section 5 will be devoted to the proof of Lemma 5.1. The proof will be performed in the following subsections. 


\subsection{Uniform bounds independent on $\epsilon$}

We have to start by deriving uniform bounds independent of $\varepsilon$. We collect them in the following lemma:

Lemma 5.2. Let $\left(\varrho_{\varepsilon}, \mathbf{u}_{\varepsilon}\right)$ (and associated $\mathbb{Z}_{\varepsilon}$ ) be a sequence of (genegalized) solutions of the approximate problem (4.1-4.2) constructed in Lemma 4.1. Then under assumptions of Lemma 4.1 there holds:

$$
\begin{gathered}
\left\|\mathbf{u}_{\epsilon}\right\|_{L^{2}\left(I, W^{1,2}(\Omega)\right)} \leq L(\text { data }, \delta), \\
\varepsilon^{1 / 4}\left\|\mathbf{u}_{\epsilon}\right\|_{L^{4}\left(I, W^{1,4}(\Omega)\right)} \leq L(\text { data }, \delta), \\
\left\|\varrho_{\epsilon}\right\|_{L^{\infty}\left(I, L^{\beta}(\Omega)\right)} \leq L(\text { data }, \delta) \\
\left\|\varrho_{\epsilon}\left|\mathbf{u}_{\epsilon}\right|^{2}\right\|_{L^{\infty}\left(I, L^{1}(\Omega)\right)} \leq L(\text { data }, \delta), \\
\sqrt{\epsilon}\left\|\nabla \varrho_{\epsilon}\right\|_{L^{2}\left(Q_{T}\right)} \leq L(\text { data }, \delta), \\
\left\|\varrho_{\epsilon}\right\|_{L^{\beta+1}((0, T) \times K)} \leq L(\text { data }, \delta, K, \delta), \text { with any compacts } K \subset \Omega .
\end{gathered}
$$

(We recall that $\mathbb{Z}_{\varepsilon}$ converges to 0 in $L^{4 / 3}\left(Q_{T}\right)$ according to (4.80)). Here $L$ is a positive constant, which is, in particular, independent of $\epsilon$.

\section{Proof of Lemma 5.2}

Continuity equation (4.9) provides bound

$$
\int_{\Omega} \varrho_{\varepsilon}(\tau) \mathrm{d} x \leq \int_{\Omega} \varrho_{0} \mathrm{~d} x-\int_{0}^{\tau} \int_{\Gamma_{\mathrm{in}}} \varrho_{B} \mathbf{u}_{B} \cdot \mathbf{n} \mathrm{d} S_{x} \mathrm{~d} t .
$$

With this bound at hand, uniform estimates (5.6-5.10) follow directly from energy inequality (4.11), structural assumptions on pressure $p$, definitions of $p_{\delta}$ and $H_{\delta}$, see $(2.2),(4.5),(2.10)$ and (4.6), in the same manner as detailed in Sections 4.3.3 and 4.3.4. The last estimate is based on the properties of the so-called Bogovskii operator. We shall recall them in the following lemma.

Lemma 5.3. Let $\Omega$ be a bounded Lipschitz domain. Then there exists a linear operator

$$
\left.\mathscr{B}:\left\{f \in C_{c}^{\infty}\left(\Omega ; R^{3}\right) \mid \int_{\Omega} f \mathrm{~d} x=0\right\} \mapsto C_{c}^{\infty}\left(\Omega ; R^{3}\right)\right\}
$$

such that: 1) $\operatorname{div} B[f]=f$; 2) $\mathscr{B}$ is bounded linear operator from $\bar{L}^{p}(\Omega)$ to $W^{1, p}(\Omega)$ for any $1<p<\infty$ (i.e. there is $c=c(p)>0$ such that $\left.\|\mathscr{B}[f]\|_{W^{1, p}\left(\Omega ; R^{3}\right)} \leq c(p)\|f\|_{L^{p}(\Omega)}\right)$ for all $f \in \bar{L}^{p}(\Omega)$; 3) if $f=\operatorname{divg}$, $\mathbf{g} \in L^{q}(\Omega), 1<q<\infty$ with $\left.\mathbf{g} \cdot \mathbf{n}\right|_{\partial \Omega}=0$ in the sense of normal traces, then there is $c=c(q)>0$ such that $\|\mathscr{B}[f]\|_{L^{q}\left(\Omega ; R^{3}\right)} \leq c(q)\|\mathbf{g}\|_{L^{q}\left(\Omega, R^{3}\right)}$ for all $\mathbf{g}$ with the above properties. In the above $\bar{L}^{p}(\Omega):=\{f \in$ $\left.L^{p}(\Omega) \mid \int_{\Omega} f \mathrm{~d} x=0\right\}$. 
We shall obtain estimate (5.11) by taking in the momentum equation (4.10) with $\left(\varrho_{\varepsilon}, \mathbf{u}_{\varepsilon}\right)$ test function

$$
\varphi=\eta(t) \mathscr{B}\left[\psi \varrho_{\varepsilon}-\frac{1}{|\Omega|} \int_{\Omega} \psi \varrho_{\varepsilon} \mathrm{d} x\right],
$$

where $\eta \in W_{0}^{1, \infty}(0, T)$ and $\psi \in C_{c}^{1}(\Omega)$ are convenient cut off functions. This testing provides an identity of the form

$$
\int_{0}^{T} \int_{\Omega} \eta \psi p_{\delta}\left(\varrho_{\varepsilon}\right) \varrho_{\varepsilon} \mathrm{d} x \mathrm{~d} t=\int_{0}^{T} \int_{\Omega} R\left(\varrho_{\varepsilon}, \mathbf{u}_{\varepsilon}, \eta, \psi\right) \mathrm{d} x \mathrm{~d} t
$$

where the right hand side may be bounded from above via the uniform estimates (5.6-5.10) by virtue of Hölder, Sobolev and interpolation inequalities, and Lemma 5.3 by a positive number dependent of $\nabla_{x} \psi$, but independent, in particular, of $\eta, \eta^{\prime}$ (and, of course, independent of $\varepsilon$ ). In order to obtain this formula, one must perform several times integration by parts and employ conveniently continuity equation (4.9). We notice that the most disagreeable terms involving integration over the boundary vanish due to the fact that $\varphi$ and $\psi$ vanish at the boundary. This is nowadays a standard and well understood procedure. We refer the reader for more details to [11, Section 3.2], or to monographs [7], [17], [9]. Seeing decomposition (2.2) of the pressure and seeing that $\mathfrak{p}$ is bounded, the latter formula provides bound (5.11).

\subsection{Weak limits in continuity and momentum equations}

We shall first pass to the limit in the weak formulations of the continuity equation (4.9) and momentum equation (4.10). Estimates (5.6) and (5.8) yield convergence (5.1), and estimate (5.11) together with $(2.2)$, (4.5) implies $p_{\delta}\left(\varrho_{\varepsilon}\right) \rightarrow \overline{p_{\delta}(\varrho)}$ weakly in $\left.L^{\frac{\beta+1}{\beta}}((0, T) \times K)\right)$ for any compact $K \subset \Omega$. Here, and in the sequel, $\overline{g(\varrho, \mathbf{u})}$ denotes a weak limit in $L^{1}\left(Q_{T}\right)$ of the sequence $g\left(\varrho_{\varepsilon}, \mathbf{u}_{\varepsilon}\right)$. By virtue of $(5.10)($ and $(5.6))$ the terms multiplied by $\varepsilon$ will vanish in the limit. Sequence $\mathbb{Z}_{\varepsilon} \rightarrow 0$ in $L^{4 / 3}\left(Q_{T} ; R^{9}\right)$ by construction, see (4.80). Seeing that $\varrho_{\varepsilon} \rightarrow \varrho$ in $C_{\text {weak }}\left([0, T] ; L^{\beta}(\Omega)\right.$ ) (as one can show by means of the Arzela-Ascoli type argument from equation (4.9) and uniform bounds (5.6-5.10)), we deduce from the compact imbedding $L^{\beta}(\Omega) \hookrightarrow \hookrightarrow W^{-1,2}(\Omega)$ and from $\mathbf{u}_{\varepsilon} \rightarrow \mathbf{u}$ in $L^{2}\left(0, T ; W^{1,2}(\Omega)\right)$ the weak- ${ }^{*}$ convergence $\varrho_{\varepsilon} \mathbf{u}_{\varepsilon} \longrightarrow_{*} \varrho \mathbf{u}$ in $L^{\infty}\left(0, T ; L^{\frac{2 \beta}{\beta+1}}(\Omega)\right)$ that may be consequently improved thanks to momentum equation $(4.10)$ and estimates $(5.6-5.10)$ to $\varrho_{\varepsilon} \mathbf{u}_{\varepsilon} \rightarrow \varrho \mathbf{u}$ in $C_{\text {weak }}\left(0, T ; L^{\frac{2 \beta}{\beta+1}}(\Omega)\right)$ again by the Arzela-Ascoli type argument. With this observation at hand, employing compact imbedding $L^{\frac{2 \beta}{\beta+1}}(\Omega) \hookrightarrow \hookrightarrow W^{-1,2}(\Omega)$ and $\mathbf{u}_{\varepsilon} \rightarrow \mathbf{u}$ in $L^{2}\left(0, T ; W^{1,2}(\Omega)\right)$ we infer that $\varrho_{\varepsilon} \mathbf{u}_{\varepsilon} \rightarrow \varrho \mathbf{u}$ in $L^{2}\left(0, T, W^{-1,2}\left(\Omega ; R^{3}\right)\right)$ and consequently $\varrho_{\varepsilon} \mathbf{u}_{\varepsilon} \otimes \mathbf{u}_{\varepsilon} \rightarrow \varrho \mathbf{u} \otimes \mathbf{u}$ weakly e.g. in $L^{1}\left(Q_{T} ; R^{9}\right)$, at least for a chosen subsequence (not relabeled). Having the above, we get the following limits in equations (4.9-4.10):

$$
\begin{gathered}
\int_{\Omega} \varrho(\tau, x) \varphi(\tau, x) \mathrm{d} x-\int_{\Omega} \varrho_{0}(x) \varphi(0, x) \mathrm{d} x=\int_{0}^{\tau} \int_{\Omega}\left(\varrho \partial_{t} \varphi+\varrho \mathbf{u} \cdot \nabla_{x} \varphi\right) \mathrm{d} x \mathrm{~d} t \\
=-\int_{0}^{\tau} \int_{\Gamma_{\mathrm{in}}} \varrho_{B} \mathbf{u}_{B} \cdot \mathbf{n} \varphi \mathrm{d} S_{x} \mathrm{~d} t
\end{gathered}
$$


for any $\tau \in[0, T]$ and $\varphi \in C_{c}^{1}\left([0, T] \times\left(\Omega \cup \Gamma_{\text {in }}\right)\right)$;

$$
\begin{gathered}
\int_{\Omega} \varrho \mathbf{u}(\tau, \cdot) \cdot \boldsymbol{\varphi}(\tau, \cdot) \mathrm{d} x-\int_{\Omega} \varrho_{0} \mathbf{u}_{0}(\cdot) \varphi(0, \cdot) \mathrm{d} x \\
=\int_{0}^{\tau} \int_{\Omega}\left(\varrho \mathbf{u} \partial_{t} \varphi+\varrho \mathbf{u} \otimes \mathbf{u}: \nabla_{x} \boldsymbol{\varphi}+\overline{p_{\delta}(\varrho)} \operatorname{div}_{x} \varphi-\mathbb{S}\left(\nabla_{x} \mathbf{u}\right): \nabla_{x} \varphi\right) \mathrm{d} x \mathrm{~d} t
\end{gathered}
$$

for any $\tau \in[0, T]$ and any $\varphi \in C_{c}^{1}\left([0, T] \times \Omega ; R^{3}\right)$.

It remains to show that

$$
\overline{p_{\delta}(\varrho)}=p_{\delta}(\varrho)
$$

The rest of this section is devoted to the proof of this identity. This is equivalent to show that $\varrho_{\varepsilon} \rightarrow \varrho$ a.e. in $Q_{T}$.

\subsection{Effective viscous flux identity}

We denote by $\nabla_{x} \Delta^{-1}$ the pseudodifferential operator with Fourier symbol $\frac{\mathrm{i} \xi}{|\xi|^{2}}$ and by $\mathscr{R}$ the Riesz transform with Fourier symbol $\frac{\xi \otimes \xi}{|\xi|^{2}}$. Following Lions [14], we shall use in the approximating momentum equation (4.9) test function

$$
\varphi(t, x)=\psi(t) \phi(x) \nabla_{x} \Delta^{-1}\left(\varrho_{\varepsilon} \phi\right), \quad \psi \in C_{c}^{1}(0, T), \phi \in C_{c}^{1}(\Omega)
$$

and in the limiting momentum equation (5.13) test function

$$
\varphi(t, x)=\psi(t) \phi(x) \nabla_{x} \Delta^{-1}(\varrho \phi), \quad \psi \in C_{c}^{1}(0, T), \phi \in C_{c}^{1}(\Omega)
$$

subtract both identities and perform the limit $\varepsilon \rightarrow 0$. This is a laborious, but nowadays standard calculation (whose details can be found e.g. in [11, Lemma 3.2], [17], [7] or [9, Chapter 3]) leading to the identity

$$
\begin{gathered}
\int_{0}^{T} \int_{\Omega} \psi \phi^{2}\left(\overline{p_{\delta}(\varrho)}-(2 \mu+\lambda) \operatorname{div} \mathbf{u}\right) \varrho \mathrm{d} x \mathrm{~d} t-\int_{0}^{T} \int_{\Omega} \psi \phi^{2}\left(\overline{p_{\delta}(\varrho) \varrho}-(2 \mu+\lambda) \overline{\varrho \mathrm{div} u}\right) \mathrm{d} x \mathrm{~d} t \\
=\int_{0}^{T} \int_{\Omega} \psi \phi \mathbf{u} \cdot(\varrho \mathscr{R} \cdot(\varrho \mathbf{u} \phi)-\varrho \mathbf{u} \cdot \mathscr{R}(\varrho \phi)) \mathrm{d} x \mathrm{~d} t \\
-\lim _{\varepsilon \rightarrow 0} \int_{0}^{T} \int_{\Omega} \psi \phi \mathbf{u}_{\varepsilon} \cdot\left(\varrho_{\varepsilon} \mathscr{R} \cdot\left(\varrho_{\varepsilon} \mathbf{u}_{\varepsilon} \phi\right)-\varrho_{\varepsilon} \mathbf{u}_{\varepsilon} \cdot \mathscr{R}\left(\varrho_{\varepsilon} \phi\right)\right) \mathrm{d} x \mathrm{~d} t .
\end{gathered}
$$

This process involves several integrations by parts and exploits continuity equation in form (4.9) and (5.12). We notice that the non homogenous data do not play any role due to the presence of compactly supported cut-off functions $\psi$ and $\phi$. The essential observation for getting (5.14) is the fact that the map $\varrho \rightarrow \varphi$ defined above is a linear and continuous from $L^{p}(\Omega)$ to $W^{1, p}(\Omega), 1<p<\infty$ as a consequence of classical Hörmander-Michlin's multiplier theorem of harmonic analysis. The most non trivial moment 
in this process is to show that the right hand side of identity (5.14) is 0 . To see it (we repeat the reasoning [11] for the sake of completeness) we first realize that the $C_{\text {weak }}\left([0, T], L^{\beta}(\Omega)\right)$-convergence of $\varrho_{\varepsilon}$ and $C_{\text {weak }}\left([0, T], L^{\frac{2 \beta}{\beta+1}}(\Omega)\right)$-convergence of $\varrho_{\varepsilon} \mathbf{u}_{\varepsilon}$ evoked in Section 5.2 imply, in particular,

$$
\text { for all } t \in[0, T], \varrho_{\varepsilon}(t) \rightarrow \varrho(t) \text { in e.g. } L^{\beta}(\Omega) \varrho_{\varepsilon} \mathbf{u}_{\varepsilon}(t) \rightarrow \varrho \mathbf{u}(t) \text { in } L^{\frac{2 \beta}{\beta+1}}(\Omega) .
$$

Since $\mathscr{R}$ is a continuous operator from $L^{p}\left(R^{3}\right)$ to $L^{p}\left(R^{3}\right), 1<p<\infty$, we also have

$$
\text { for all } t \in[0, T], \mathscr{R}\left[\varrho_{\varepsilon}(t) \phi\right] \rightarrow \mathscr{R}[\varrho(t) \phi] \text { in } L^{\beta}(\Omega), \quad \mathscr{R}\left[\varrho_{\varepsilon} \mathbf{u}_{\varepsilon}(t) \phi\right] \rightarrow \mathscr{R}[\varrho \mathbf{u}(t) \phi] \text { in } L^{\frac{2 \beta}{\beta+1}}(\Omega) .
$$

At this stage we report a convenient version of the celebrated Div-Curl lemma, see [7, Section 6] or [9, Theorem 10.27]. It reads

Lemma 5.4. Let

$$
\begin{aligned}
& \mathbf{V}_{\varepsilon} \rightarrow \mathbf{V} \text { weakly in } L^{p}\left(\mathbb{R}^{N} ; \mathbb{R}^{N}\right), \\
& \mathbf{U}_{\varepsilon} \rightarrow \mathbf{U} \text { weakly in } L^{q}\left(\mathbb{R}^{N} ; \mathbb{R}^{N}\right),
\end{aligned}
$$

where $\frac{1}{p}+\frac{1}{q}=\frac{1}{s}<1$. Then

$$
\mathbf{U}_{\varepsilon} \cdot \mathscr{R}\left[\mathbf{V}_{\varepsilon}\right]-\mathscr{R}\left[\mathbf{U}_{\varepsilon}\right] \cdot \mathbf{V}_{\varepsilon} \rightarrow \mathbf{U} \cdot \mathscr{R}[\mathbf{V}]-\mathscr{R}[\mathbf{U}] \cdot \mathbf{V} \text { weakly in } L^{s}\left(\mathbb{R}^{N}\right) .
$$

Applying this lemma to the above situation, we get

$$
\text { for all } t \in[0, T],\left[\varrho_{\varepsilon} \mathscr{R} \cdot\left(\varrho_{\varepsilon} \mathbf{u}_{\varepsilon} \phi\right)-\varrho_{\varepsilon} \mathbf{u}_{\varepsilon} \cdot \mathscr{R}\left(\varrho_{\varepsilon} \phi\right)\right](t) \rightarrow[\varrho \mathscr{R} \cdot(\varrho \mathbf{u} \phi)-\varrho \mathbf{u} \cdot \mathscr{R}(\varrho \phi)](t) \text { in } L^{\frac{2 \beta}{\beta+3}}(\Omega) \text {. }
$$

In view of compact imbedding $L^{\frac{2 \beta}{\beta+3}}(\Omega) \hookrightarrow \hookrightarrow W^{-1,2}(\Omega)$, we have also

for all $t \in[0, T],\left[\varrho_{\varepsilon} \mathscr{R} \cdot\left(\varrho_{\varepsilon} \mathbf{u}_{\varepsilon} \phi\right)-\varrho_{\varepsilon} \mathbf{u}_{\varepsilon} \cdot \mathscr{R}\left(\varrho_{\varepsilon} \phi\right)\right](t) \rightarrow[\varrho \mathscr{R} \cdot(\varrho \mathbf{u} \phi)-\varrho \mathbf{u} \cdot \mathscr{R}(\varrho \phi)](t)$ in $W^{-1,2}(\Omega)$.

We easily verify that the sequence $\left\|\varrho_{\varepsilon} \mathscr{R} \cdot\left(\varrho_{\varepsilon} \mathbf{u}_{\varepsilon}\right)-\varrho_{\varepsilon} \mathbf{u}_{\varepsilon} \cdot \mathscr{R}\left(\varrho_{\varepsilon}\right)\right\|_{W^{-1,2}(\Omega)}$ is bounded in $L^{\infty}(0, T)$; whence the latter convergence yields the strong convergence

$$
\varrho_{\varepsilon} \mathscr{R} \cdot\left(\varrho_{\varepsilon} \mathbf{u}_{\varepsilon} \phi\right)-\varrho_{\varepsilon} \mathbf{u}_{\varepsilon} \cdot \mathscr{R}\left(\varrho_{\varepsilon} \phi\right) \rightarrow\left[\varrho \mathscr{R} \cdot(\varrho \mathbf{u} \phi)-\varrho \mathbf{u} \cdot \mathscr{R}(\varrho \phi) \text { in } L^{2}\left(0, T ; W^{-1,2}(\Omega)\right) .\right.
$$

Recalling the $L^{2}\left(0, T ; W^{1,2}(\Omega)\right)$-weak convergence of $\mathbf{u}_{\varepsilon}$ we get the desired result. Identity (5.14) now reads

$$
\overline{p(\varrho) \varrho}-\overline{p(\varrho)} \varrho=(2 \mu+\lambda)(\overline{\varrho \operatorname{div} u}-\varrho \operatorname{div} \mathbf{u})
$$

If the pressure were non decreasing (i.e. if $\mathfrak{p}$ would be identically zero), we would have by Miniti's trick, $\overline{p(\varrho) \varrho}-\overline{p(\varrho)} \varrho \geq 0$ a.e. in $Q_{T}$, see [9, Theorem 10.19] and consequently $\overline{\varrho \operatorname{divu}}-\varrho \operatorname{div} \mathbf{u} \geq 0$ a.e. in $Q_{T}$. We however consider a non-monotone pressure and this simple conclusion is not true anymore.

We have to further extend this argument. Following Feireisl [7], we realize that there is $\Lambda>0$ (dependent on $\mathfrak{p}$ ) such that

$$
[0, \infty) \ni \varrho \mapsto \Lambda \varrho \ln \varrho-\varrho \mathfrak{p}(\varrho), \quad[0, \infty) \ni \varrho \mapsto \Lambda \varrho \ln \varrho+\mathfrak{p}(\varrho)
$$


are convex. Consequently,

$$
\Lambda(\overline{\varrho \ln \varrho}-\varrho \ln \varrho) \geq \overline{\varrho \mathfrak{p}(\varrho)}-\varrho \mathfrak{p}(\varrho)
$$

and

$$
\Lambda(\overline{\varrho \ln \varrho}-\varrho \ln \varrho) \geq \mathfrak{p}(\varrho)-\overline{\mathfrak{p}(\varrho)} .
$$

Coming now back to (5.15), we obtain by using monotonicity of $p$,

$$
\begin{gathered}
(2 \mu+\lambda)(\varrho \operatorname{div} \mathbf{u}-\overline{\varrho \operatorname{div} u}) \leq \overline{\varrho \mathfrak{p}(\varrho)}-\varrho \overline{\mathfrak{p}(\varrho)} \\
\leq[\overline{\varrho \mathfrak{p}(\varrho)}-\varrho \mathfrak{p}(\varrho)]+\varrho[\mathfrak{p}(\varrho)-\overline{\mathfrak{p}(\varrho)}] .
\end{gathered}
$$

Employing (5.17) and (5.18) further yields

$$
\varrho \operatorname{div} \mathbf{u}-\overline{\varrho \operatorname{div} u} \leq c \Lambda(1+\bar{r})(\overline{\varrho \ln \varrho}-\varrho \ln \varrho),
$$

provided supp $\mathfrak{p} \subset[0, \bar{r}]$. This is the crucial inequality that plays in the case of non monotone pressure the same role as would be played by the inequality $\overline{\varrho \operatorname{div} u}-\varrho \operatorname{div} \mathbf{u} \geq 0$ in the case of monotone pressure law.

\subsection{Strong convergence of density sequence}

Since $\varrho$ verifies continuity equation (5.12) and since it belongs to to $L^{2}\left(Q_{T}\right)$ we may employ Lemma 3.1 in order to conclude that it verifies also renormalized continuity equation (2.13). In view of Remark 2.5 , identity (2.13) is valid for any $b$ belonging to class (2.14). In particular, for $b(\varrho) \equiv L(\varrho)=\varrho \log \varrho$, it reads

$$
\begin{gathered}
\int_{\Omega} L(\varrho(\tau, x)) \varphi(\tau, x) \mathrm{d} x-\int_{\Omega} L\left(\varrho_{0}\right) \varphi(0, x) \mathrm{d} x \\
=\int_{0}^{\tau} \int_{\Omega}\left(L(\varrho) \partial_{t} \varphi+L(\varrho) \mathbf{u} \cdot \nabla_{x} \varphi-\varphi \varrho \operatorname{div}_{x} \mathbf{u}\right) \mathrm{d} x \mathrm{~d} t+\int_{0}^{\tau} \int_{\partial \Omega} L\left(\varrho_{B}\right) \mathbf{u}_{B} \cdot \mathbf{n} \varphi \mathrm{d} S_{x} \mathrm{~d} t .
\end{gathered}
$$

We continue with the renormalized version of the approximate equation of continuity (4.16). In particular, for $b(\varrho)=L(\varrho) \equiv \varrho \log (\varrho)$, when passing to the weak formulation, we obtain

$$
\begin{gathered}
\int_{\Omega} L\left(\varrho_{\varepsilon}(\tau, x)\right) \varphi(\tau, x) \mathrm{d} x-\int_{\Omega} L\left(\varrho_{0}(x)\right) \varphi(0, x) \mathrm{d} x \\
-\int_{0}^{\tau} \int_{\Omega}\left(L\left(\varrho_{\varepsilon}\right) \partial_{t} \varphi+L\left(\varrho_{\varepsilon}\right) \mathbf{u}_{\varepsilon} \cdot \nabla_{x} \varphi\right) \mathrm{d} x \mathrm{~d} t+\int_{0}^{\tau} \int_{\Omega} \varrho_{\varepsilon} \operatorname{div}_{x} \mathbf{u}_{\varepsilon} \varphi \mathrm{d} x \mathrm{~d} t \\
+\int_{0}^{\tau} \int_{\Gamma_{\text {in }}} \varphi L\left(\varrho_{\varepsilon}\right) \mathbf{u}_{B} \cdot \mathbf{n} \mathrm{d} S_{x} \mathrm{~d} t-\varepsilon \int_{0}^{\tau} \int_{\Gamma_{\text {in }}} \varphi L^{\prime}\left(\varrho_{\varepsilon}\right) \nabla_{x} \varrho_{\varepsilon} \cdot \mathbf{n} \mathrm{d} S_{x} \mathrm{~d} t \leq o(\varepsilon)
\end{gathered}
$$


for any $\tau \in[0, T]$ and any $\varphi \in C_{c}^{1}\left([0, T] \times\left(\Omega \cup \Gamma_{\text {in }}\right)\right), \varphi \geq 0$, where the inequality sign appears due to the omission of the (non negative) term containing $\varepsilon L^{\prime \prime}\left(\varrho_{\varepsilon}\right)\left|\nabla_{x} \varrho_{\varepsilon}\right|^{2}$ (recall that $L$ is convex) and $o(\varepsilon)$, $\lim _{\varepsilon \rightarrow 0} O(\varepsilon)=0$ corresponds to the terms of (4.16) containing $\varepsilon$ as multiplier.

Finally, we use the boundary conditions (4.10) obtaining

$$
\begin{gathered}
\int_{\Omega} L\left(\varrho_{\varepsilon}(\tau, x)\right) \varphi(\tau, x) \mathrm{d} x-\int_{\Omega} L\left(\varrho_{0}(x)\right) \varphi(0, x) \mathrm{d} x \\
-\int_{0}^{\tau} \int_{\Omega}\left(L\left(\varrho_{\varepsilon}\right) \partial_{t} \varphi+L\left(\varrho_{\varepsilon}\right) \mathbf{u}_{\varepsilon} \cdot \nabla_{x} \varphi\right) \mathrm{d} x \mathrm{~d} t+\int_{0}^{\tau} \int_{\Omega} \varrho_{\varepsilon} \operatorname{div}{ }_{x} \mathbf{u}_{\varepsilon} \varphi \mathrm{d} x \mathrm{~d} t \\
+\int_{0}^{\tau} \int_{\Gamma_{\mathrm{in}}}\left(L\left(\varrho_{\varepsilon}\right) \mathbf{u}_{B} \cdot \mathbf{n}+L^{\prime}\left(\varrho_{\varepsilon}\right)\left(\varrho_{B}-\varrho_{\varepsilon}\right) \mathbf{u}_{B} \cdot \mathbf{n}\right) \mathrm{d} S_{x} \mathrm{~d} t \leq o(\varepsilon)
\end{gathered}
$$

for any $\tau \in[0, T]$ and any $\varphi \in C_{c}^{1}\left([0, T] \times\left(\Omega \cup \Gamma_{\text {in }}\right)\right), \varphi \geq 0$.

Subtracting (5.20) from (5.22) while taking $\varphi$ independent of $t \in[0, T]$, we get

$$
\begin{gathered}
\int_{\Omega} L\left(\varrho_{\varepsilon}(\tau, x)\right) \varphi(x) \mathrm{d} x-\int_{\Omega} L(\varrho(\tau, x)) \varphi(x) \mathrm{d} x \\
-\int_{0}^{\tau} \int_{\Omega}\left(L\left(\varrho_{\varepsilon}\right)-L(\varrho)\right) \mathbf{u}_{\varepsilon} \cdot \nabla_{x} \varphi \mathrm{d} x \mathrm{~d} t+\int_{0}^{\tau} \int_{\Omega}\left(\varrho_{\varepsilon} \operatorname{div}_{x} \mathbf{u}_{\varepsilon}-\varrho \operatorname{div}_{x} \mathbf{u}\right) \varphi \mathrm{d} x \mathrm{~d} t \\
+\int_{0}^{\tau} \int_{\Gamma_{\mathrm{in}}} \varphi\left[L\left(\varrho_{B}\right)-L^{\prime}\left(\varrho_{\varepsilon}\right)\left(\varrho_{B}-\varrho_{\varepsilon}\right)-L\left(\varrho_{\varepsilon}\right)\right]\left|\mathbf{u}_{B} \cdot \mathbf{n}\right| \mathrm{d} S_{x} \leq o(\varepsilon)
\end{gathered}
$$

for any $\tau \in[0, T]$ and any $\left.\varphi \in C_{c}^{1}\left(\Omega \cup \Gamma_{\text {in }}\right)\right), \varphi \geq 0$. As $L$ is convex, we deduce,

$$
\begin{gathered}
\int_{\Omega} L\left(\varrho_{\varepsilon}(\tau, x)\right) \varphi(x) \mathrm{d} x-\int_{\Omega} L(\varrho(\tau, x)) \varphi(x) \mathrm{d} x \\
-\int_{0}^{\tau} \int_{\Omega}\left(L\left(\varrho_{\varepsilon}\right)-L(\varrho)\right) \mathbf{u}_{\varepsilon} \cdot \nabla_{x} \varphi \mathrm{d} x \mathrm{~d} t+\int_{0}^{\tau} \int_{\Omega}\left(\varrho_{\varepsilon} \operatorname{div}_{x} \mathbf{u}_{\varepsilon}-\varrho \operatorname{div}_{x} \mathbf{u}\right) \varphi \mathrm{d} x \mathrm{~d} t \leq o(\varepsilon) .
\end{gathered}
$$

Whence, letting $\varepsilon \rightarrow 0$ yields

$$
\begin{gathered}
\int_{\Omega}(\overline{\varrho \log \varrho}-\varrho \log \varrho)(\tau, x) \varphi(x) \mathrm{d} x+\int_{0}^{\tau} \int_{\Omega}(\varrho \log \varrho-\overline{\varrho \log \varrho}) \mathbf{u} \cdot \nabla_{x} \varphi \mathrm{d} x \mathrm{~d} t \\
\leq c \Lambda(1+\bar{r}) \int_{0}^{\tau} \int_{\Omega}(\overline{\varrho \log \varrho}-\varrho \log \varrho) \mathrm{d} x \mathrm{~d} t
\end{gathered}
$$

for any $\tau \in[0, T]$ and any $\varphi \in C_{c}^{1}\left(\Omega \cup \Gamma_{\text {in }}\right), \varphi \geq 0$, where we have used (5.19).

Let now $\tilde{\mathbf{u}}_{B} \in W^{1, \infty}(\Omega)$ be a Lipschitz extension of $\mathbf{u}_{B}$ to $\Omega$ constructed in Lemma 2.2. Since $\Gamma_{\text {out }}$ is in class $C^{2}$, function $x \mapsto \operatorname{dist}\left(x, \Gamma_{\text {out }}\right)$ belongs to $C^{2}\left(\mathcal{U}_{\varepsilon_{0}}^{-}\left(\Gamma_{\text {out }}\right) \cup \Gamma_{\text {out }}\right)$ for some "small"' $\varepsilon_{0}>0$, where

$$
\mathcal{U}_{\varepsilon_{0}}^{-}\left(\Gamma_{\text {out }}\right) \equiv\left\{x=\mathbf{x}_{0}-z \mathbf{n}\left(\mathbf{x}_{0}\right) \mid x_{0} \in \Gamma_{\text {out }}, 0<z<\varepsilon_{0}\right\} \cap \Omega
$$


cf. Foote [12]. Moreover,

$$
\nabla_{x} \operatorname{dist}\left(x, \Gamma_{\text {out }}\right) \rightarrow-\mathbf{n}\left(\mathbf{x}_{0}\right) \text {, whenever } \mathscr{U}_{\varepsilon}^{-}\left(\Gamma_{\text {out }}\right) \ni x \rightarrow \mathbf{x}_{0} \in \Gamma_{\text {out }}, 0<\varepsilon<\varepsilon_{0} .
$$

Therefore,

$$
\tilde{\mathbf{u}}_{B} \cdot \nabla_{x} \operatorname{dist}\left(x, \Gamma_{\text {out }}\right)<0 \text { for all } x \in \mathcal{U}_{\varepsilon}^{-}\left(\Gamma_{\text {out }}\right) \text { and } 0<\varepsilon<\varepsilon_{0} .
$$

Since $\Omega$ is Lipschitz, we have also that

$$
\left|\hat{\mathcal{U}}_{\varepsilon}^{-}\left(\Gamma_{\text {out }}\right) \Delta \mathcal{U}_{\varepsilon}^{-}\left(\Gamma_{\text {out }}\right)\right| \rightarrow 0 \text { as } \varepsilon \rightarrow 0,
$$

where

$$
\hat{\mathcal{U}}_{\varepsilon}^{-}\left(\Gamma_{\text {out }}\right) \equiv\left\{x \in \Omega \mid \operatorname{dist}\left(x, \Gamma_{\text {out }}\right)<\varepsilon\right\}
$$

and $A \Delta B$ denotes the symmetric difference of sets $A$ and $B$.

Consider family of Lipschitz test functions in $\Omega$,

$$
\varphi_{\varepsilon}(x)=\left\{\begin{array}{l}
1 \text { if } \operatorname{dist}\left(x, \Gamma_{\text {out }}\right)>\varepsilon \\
\frac{1}{\varepsilon} \operatorname{dist}\left(x, \Gamma_{\text {out }}\right) \text { if } \operatorname{dist}\left(x, \Gamma_{\text {out }}\right) \leq \varepsilon
\end{array}\right\}
$$

By Lebesgue theorem and Hardy's inequality (we notice that $\left|\nabla_{x} \varphi_{\varepsilon}(x)\right| \leq \frac{1}{\varepsilon}$ ),

$$
\int_{0}^{T} \int_{\Omega}[\varrho \log (\varrho)-\overline{\varrho \log (\varrho)}]\left(\mathbf{u}-\tilde{\mathbf{u}}_{B}\right) \cdot \nabla_{x} \varphi_{\varepsilon} \mathrm{d} x \mathrm{~d} t \rightarrow 0 \text { as } \varepsilon \rightarrow 0,
$$

while, in accordance with (5.25), (5.26)

$$
\liminf _{\varepsilon \rightarrow 0} \int_{0}^{T} \int_{\Omega}[\varrho \log (\varrho)-\overline{\varrho \log (\varrho)}] \tilde{\mathbf{u}}_{B} \cdot \nabla_{x} \varphi_{\varepsilon} \mathrm{d} x \mathrm{~d} t \geq 0
$$

Coming with this information back to (5.24) we conclude that

$$
\int_{\Omega}(\overline{\varrho \log \varrho}-\varrho \log \varrho)(\tau, x) \mathrm{d} x \leq c \Lambda(1+\bar{r}) \int_{0}^{\tau} \int_{\Omega}(\overline{\varrho \log \varrho}-\varrho \log \varrho) \mathrm{d} x \mathrm{~d} t, \tau \in[0, T]
$$

whence by the Gronwall lemma

$$
\int_{\Omega}(\overline{\varrho \log \varrho}-\varrho \log \varrho)(\tau, x) \mathrm{d} x \leq 0
$$

On the other hand, since $L$ is convex, $\overline{\varrho \log \varrho}-\varrho \log \varrho \geq 0$ a.e. in $Q_{T}$. Inequality (5.30) thus yields

$$
\overline{\varrho \log \varrho}=\varrho \log \varrho \text { a.e. in } Q_{T} \text {; }
$$

Consequently

$$
\varrho_{\varepsilon} \rightarrow \varrho \text { a.e. in } Q_{T} \text { and strongly in } L^{p}((0, T) \times K) \text { and in } L^{s}\left(Q_{T}\right)
$$

with any compact $K \subset \Omega, p \in[1, \beta+1), s \in[1, \beta)$, see e.g. [9, Theorem 10.20]. Therefore $\overline{p_{\delta}(\varrho)}=p_{\delta}(\varrho)$ in equation (5.13). We have proved identity (5.4)

Lemma 5.1 is almost proved. It remains to show the energy inequality (5.5). This will be done in the next section. 


\subsection{Energy inequality}

We shall pass to the limit in the energy inequality (4.11) with the goal to deduce from it energy inequality (5.5). To this end we first take identity (4.16) with $b(z)=z^{2}, \varphi=1$ in order to get identity $(4.41)_{\left(\varrho_{\varepsilon}, \mathbf{u}_{\varepsilon}\right)}$. This is justified by virtue of Remark 4.2. Due to this operation, all terms in (4.11) multiplied by $\Sigma$ vanish.

Once this is done, we integrate the resulting inequality over $\tau$ from $0<\tau_{1}<\tau_{2}<T$ to get

$$
\begin{aligned}
\int_{\tau_{1}}^{\tau_{2}} \int_{\Omega}\left(\frac{1}{2} \varrho_{\varepsilon}\left|\mathbf{u}_{\varepsilon}-\mathbf{u}_{\infty}\right|^{2}+H_{\delta}\left(\varrho_{\varepsilon}\right)\right)(\tau) \mathrm{d} x \mathrm{~d} t+\int_{\tau_{1}}^{\tau_{2}} \int_{0}^{\tau} \int_{\Omega}\left(\mathbb{S}\left(\nabla_{x}\left(\mathbf{u}_{\varepsilon}-\mathbf{u}_{\infty}\right)\right): \nabla_{x}\left(\mathbf{u}_{\varepsilon}-\mathbf{u}_{\infty}\right) \mathrm{d} x \mathrm{~d} t\right. \\
\leq \int_{\tau_{1}}^{\tau_{2}} \int_{\Omega}\left(\frac{1}{2} \varrho_{0}\left|\mathbf{u}_{0}-\mathbf{u}_{\infty}\right|^{2}+H\left(\varrho_{0}\right)\right) \mathrm{d} x \mathrm{~d} \tau-\int_{\tau_{1}}^{\tau_{2}} \int_{0}^{\tau} \int_{\Gamma_{\mathrm{in}}} H_{\delta}\left(\varrho_{B}\right) \mathbf{u}_{B} \cdot \mathbf{n} \mathrm{d} S_{x} \mathrm{~d} t \mathrm{~d} \tau \\
\quad-(\underline{\mathfrak{E}}-\delta B) \int_{0}^{\tau} \int_{\Gamma_{\text {in }}}\left|\mathbf{u}_{B} \cdot \mathbf{n}\right| \mathrm{d} S_{x} \mathrm{~d} t-(\underline{H}-\delta A) \int_{0}^{\tau} \int_{\Gamma_{\text {out }}}\left|\mathbf{u}_{B} \cdot \mathbf{n}\right| \mathrm{d} S_{x} \mathrm{~d} t \\
-\int_{\tau_{1}}^{\tau_{2}} \int_{0}^{\tau} \int_{\Omega} p_{\delta}\left(\varrho_{\varepsilon}\right) \operatorname{div} \mathbf{u}_{\infty} \mathrm{d} x \mathrm{~d} t-\int_{\tau_{1}}^{\tau_{2}} \int_{0}^{\tau} \int_{\Omega} \varrho_{\varepsilon} \mathbf{u}_{\varepsilon} \cdot \nabla_{x} \mathbf{u}_{\infty} \cdot\left(\mathbf{u}_{\varepsilon}-\mathbf{u}_{\infty}\right) \mathrm{d} x \mathrm{~d} t \mathrm{~d} \tau \\
-\int_{\tau_{1}}^{\tau_{2}} \int_{0}^{\tau} \int_{\Omega}\left(\mathbb{S}\left(\nabla_{x} \mathbf{u}_{\infty}\right): \nabla_{x}\left(\mathbf{u}_{\varepsilon}-\mathbf{u}_{\infty}\right)-\varepsilon \nabla_{x} \varrho_{\varepsilon} \cdot \nabla_{x}\left(\mathbf{u}_{\varepsilon}-\mathbf{u}_{\infty}\right) \cdot \mathbf{u}_{\infty}\right) \mathrm{d} x \mathrm{~d} t \mathrm{~d} \tau
\end{aligned}
$$

where, at the left hand side we have omitted the non negative terms multiplied by $\varepsilon$ and the non negative terms involving integrals over the $\Gamma_{\text {in }}$ and $\Gamma_{\text {out }}$ portions of the boundary.

We can now use the convergences established in Section 5.2 and in (5.31) in combination with the lower weak semi-continuity of convex functionals at the left hand side (see e.g. [9, Theorem 10.20]) - to this end we write $H_{\delta}=\mathscr{H}_{\delta}+\mathfrak{H}$ and realize that $\mathscr{H}_{\delta}$ is convex and $\mathfrak{H}$ is bounded on $(0, \infty)$ - to get

$$
\begin{gathered}
\int_{\tau_{1}}^{\tau_{2}} \int_{\Omega}\left(\frac{1}{2} \varrho\left|\mathbf{u}-\mathbf{u}_{\infty}\right|^{2}+H_{\delta}(\varrho)\right)(\tau) \mathrm{d} x \mathrm{~d} t+\int_{\tau_{1}}^{\tau_{2}} \int_{0}^{\tau} \int_{\Omega}\left(\mathbb{S}\left(\nabla_{x}\left(\mathbf{u}-\mathbf{u}_{\infty}\right)\right): \nabla_{x}\left(\mathbf{u}-\mathbf{u}_{\infty}\right) \mathrm{d} x \mathrm{~d} t\right. \\
\leq \int_{\tau_{1}}^{\tau_{2}} \int_{\Omega}\left(\frac{1}{2} \varrho_{0}\left|\mathbf{u}_{0}-\mathbf{u}_{\infty}\right|^{2}+H\left(\varrho_{0}\right)\right) \mathrm{d} x \mathrm{~d} \tau-\int_{\tau_{1}}^{\tau_{2}} \int_{0}^{\tau} \int_{\Gamma_{\text {in }}} H_{\delta}\left(\varrho_{B}\right) \mathbf{u}_{B} \cdot \mathbf{n} \mathrm{d} S_{x} \mathrm{~d} t \mathrm{~d} \tau \\
-(\underline{\underline{E}}-\delta B) \int_{0}^{\tau} \int_{\Gamma_{\mathrm{in}}}\left|\mathbf{u}_{B} \cdot \mathbf{n}\right| \mathrm{d} S_{x} \mathrm{~d} t-(\underline{H}-\delta A) \int_{0}^{\tau} \int_{\Gamma_{\text {out }}}\left|\mathbf{u}_{B} \cdot \mathbf{n}\right| \mathrm{d} S_{x} \mathrm{~d} t \\
-\liminf _{\varepsilon \rightarrow 0} \int_{\tau_{1}}^{\tau_{2}} \int_{0}^{\tau} \int_{\Omega} p_{\delta}\left(\varrho_{\varepsilon}\right) \operatorname{div} \mathbf{u}_{\infty} \mathrm{d} x \mathrm{~d} t-\int_{\tau_{1}}^{\tau_{2}} \int_{0}^{\tau} \int_{\Omega} \varrho \mathbf{u} \cdot \nabla_{x} \mathbf{u}_{\infty} \cdot\left(\mathbf{u}-\mathbf{u}_{\infty}\right) \mathrm{d} x \mathrm{~d} t \mathrm{~d} \tau \\
-\int_{\tau_{1}}^{\tau_{2}} \int_{0}^{\tau} \int_{\Omega} \mathbb{S}\left(\nabla_{x} \mathbf{u}_{\infty}\right): \nabla_{x}\left(\mathbf{u}-\mathbf{u}_{\infty}\right) \mathrm{d} x \mathrm{~d} t \mathrm{~d} \tau .
\end{gathered}
$$

We observe that due to (2.11),

$$
\int_{0}^{\tau} \int_{\hat{u}_{h}^{-}(\partial \Omega)}\left(\not 2\left(\varrho_{\varepsilon}\right)+\delta\left(\varrho_{\varepsilon}^{\beta}+\varrho_{\varepsilon}\right)\right) \operatorname{div} \mathbf{u}_{\infty} \mathrm{d} x \mathrm{~d} t \geq 0
$$


(if $h>0$ is sufficiently small) and

$$
\begin{gathered}
\liminf _{\varepsilon \rightarrow 0} \int_{\tau_{1}}^{\tau_{2}} \int_{0}^{\tau} \int_{\Omega \backslash \hat{\mathcal{u}}_{h}^{-}(\partial \Omega)}\left(\left(p\left(\varrho_{\varepsilon}\right)+\delta\left(\varrho_{\varepsilon}^{\beta}+\varrho_{\varepsilon}\right)\right) \operatorname{div} \mathbf{u}_{\infty} \mathrm{d} t \mathrm{~d} \tau\right. \\
=\int_{\tau_{1}}^{\tau_{2}} \int_{0}^{\tau} \int_{\Omega \backslash \hat{\mathcal{u}}_{h}^{-}(\partial \Omega)}\left(p(\varrho)+\delta\left(\varrho^{\beta}+\varrho\right)\right) \operatorname{div} \mathbf{u}_{\infty} \mathrm{d} x \mathrm{~d} t \mathrm{~d} \tau
\end{gathered}
$$

while

$$
\int_{0}^{\tau} \int_{\Omega} \mathfrak{p}\left(\varrho_{\varepsilon}\right) \operatorname{div} \mathbf{u}_{\infty} \mathrm{d} x \mathrm{~d} t \rightarrow \int_{0}^{\tau} \int_{\Omega} \mathfrak{p}(\varrho) \operatorname{div} \mathbf{u}_{\infty} \mathrm{d} x \mathrm{~d} t
$$

by virtue of (5.31).

Using these facts in (5.32), letting $h \rightarrow 0$ and $\tau_{1} \rightarrow \tau_{2}$ while applying the Theorem on Lebesgue points yields the desired inequality (5.5). Lemma 5.1 is thus proved.

\section{$6 \quad$ Limit $\delta \rightarrow 0$. Proof of Theorem 2.4}

Our ultimate goal is to perform limit $\delta \rightarrow 0$. We will prove the following:

Lemma 6.1. Let $\left(\varrho_{\delta}, \mathbf{u}_{\delta}\right)$ be a sequence of functions constructed in Lemma 5.1. Then there is a subsequence (not relabeled) such that

$$
\begin{gathered}
\varrho_{\delta} \rightarrow \varrho \text { weakly- }{ }^{*} \text { in } L^{\infty}\left(0, T ; L^{\gamma}(\Omega)\right), \\
\mathbf{u}_{\delta} \rightarrow \mathbf{u} \text { in } L^{2}\left(0, T ; W^{1,2}\left(\Omega ; R^{3}\right)\right)
\end{gathered}
$$

where the couple $(\varrho, \mathbf{u})$ is a weak solution of problem (1.1-1.6).

The remaining part of this section is devoted to the proof of Lemma 6.1, which is nothing but Theorem 2.4.

\subsection{Uniform estimates}

We shall start with estimates for weak solutions $\left(\varrho_{\delta}, \mathbf{u}_{\delta}\right)$ constructed in Lemma 5.1. They are collected in the following lemma.

Lemma 6.2. Let $\left(\varrho_{\delta}, \mathbf{u}_{\delta}\right)$ be a couple constructed in Lemma 5.1. Then, the following estimates hold:

$$
\begin{gathered}
\left\|\mathbf{u}_{\delta}\right\|_{L^{2}\left(I, W^{1,2}\left(\Omega ; R^{3}\right)\right)} \leq L(\text { data }) \\
\left\|\varrho_{\delta}\right\|_{L^{\infty}\left(I, L^{\gamma}(\Omega)\right)} \leq L(\text { data }) \\
\left\|\varrho_{\delta} \mathbf{u}_{\delta}^{2}\right\|_{L^{\infty}\left(I, L^{1}(\Omega)\right)} \leq L(\text { data }) \\
\delta^{1 / \beta}\left\|\varrho_{\delta}\right\|_{L^{\infty}\left(I, L^{\beta}(\Omega)\right)} \leq L(\text { data })
\end{gathered}
$$


There is $\alpha(\gamma)>0$ such that

$$
\left\|\varrho_{\delta}\right\|_{L^{\gamma+\alpha}((0, T) \times K)} \leq L(\text { data, } K) \text {, with any compact } K \subset \Omega .
$$

In the above,

$$
\text { "data" stands for } \int_{\Omega}\left(\frac{1}{2} \varrho_{0} \mathbf{u}_{0}^{2}+H\left(\varrho_{0}\right)\right) \mathrm{d} x,\left\|\mathbf{u}_{\infty}\right\|_{W^{1, \infty}(\Omega)}, \underline{\varrho}_{B}, \bar{\varrho}_{B}
$$

\section{Proof of Lemma 5.2}

Similarly as before, continuity equation $(5.12)_{\left(\varrho_{\delta}, \mathbf{u}_{\delta}\right)}$ yields $L^{\infty}\left(0, T ; L^{1}(\Omega)\right)$ bound for the sequence $\varrho_{\delta}$. Now, uniform estimates (6.2-6.5) follow directly from energy inequality (5.5), structural assumptions on the pressure $p$, and definitions of $p_{\delta}$ and $H_{\delta}$, see $(2.2),(4.5),(4.6)$, and energy inequality (5.5) by the similar (in fact more simple) reasoning as that one performed in Sections 4.3.3, 4.3.4. The last estimate, as in the previous section, is based on the properties of the Bogovskii operator introduced in Lemma 5.3. We obtain it by testing the momentum equation (5.4) with $\left(\varrho_{\delta}, \mathbf{u}_{\delta}\right)$ with test function

$$
\varphi=\eta(t) \mathscr{B}\left[\psi \varrho_{\delta}^{\alpha}-\frac{1}{|\Omega|} \int_{\Omega} \psi \varrho_{\delta}^{\alpha} \mathrm{d} x\right] \text {, where } \alpha>0 \text { is sufficiently small, }
$$

and where $\eta \in W_{0}^{1, \infty}(0, T)$ and $\psi \in C_{c}^{1}(\Omega)$ are convenient cut off functions. After several integrations by parts, using renormalized equation (5.3) (with $\left(\varrho_{\delta}, \mathbf{u}_{\delta}\right)$ and $b(\varrho)=\varrho^{\alpha}$, cf. Remark 2.3), we arrive finally at

$$
\int_{0}^{T} \int_{\Omega} \eta \psi p_{\delta}\left(\varrho_{\delta}\right) \varrho_{\delta}^{\alpha} \mathrm{d} x \mathrm{~d} t=\int_{0}^{T} \int_{\Omega} R\left(\varrho_{\delta}, \mathbf{u}_{\delta}, \eta, \psi\right) \mathrm{d} x \mathrm{~d} t
$$

where the right hand side may be bounded from above due to estimates (6.2-6.5), in the same way as in the Section 5.1. We refer the reader for more details of this standard but laborious procedure again to [11, Section 4.1], or to monographs [7], [17], [9].

\subsection{Weak limits in the field equations}

Estimates (6.2-6.3) yield immediately weak convergence announced in (6.1) and estimate (6.6) together with $(2.2)$ imply $p\left(\varrho_{\delta}\right) \rightarrow \overline{p(\varrho)}$ weakly in $\left.L^{\frac{\gamma+\alpha}{\gamma}}((0, T) \times K)\right)$ for any compact $K \subset \Omega$. The terms multiplied by $\delta$ in the momentum equation will vanish due to estimate (6.5). Repeating carefully the (standard)

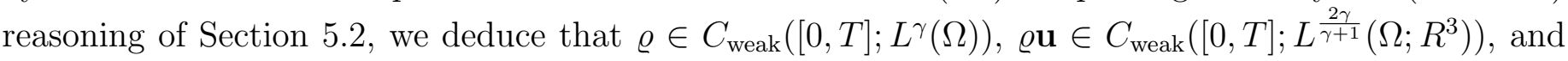
the the limit in equations (5.12) and (4.10) reads

$$
\begin{gathered}
\int_{\Omega} \varrho(\tau, \cdot) \varphi(\tau, \cdot) \mathrm{d} x-\int_{\Omega} \varrho_{0}(\cdot) \varphi(0, \cdot) \mathrm{d} x=\int_{0}^{\tau} \int_{\Omega}\left(\varrho \partial_{t} \varphi+\varrho \mathbf{u} \cdot \nabla_{x} \varphi\right) \mathrm{d} x \mathrm{~d} t \\
=-\int_{0}^{\tau} \int_{\Gamma_{\mathrm{in}}} \varrho_{B} \mathbf{u}_{B} \cdot \mathbf{n} \varphi \mathrm{d} S_{x} \mathrm{~d} t
\end{gathered}
$$

for any $\tau \in[0, T]$ and $\varphi \in C_{c}^{1}\left([0, T] \times\left(\Omega \cup \Gamma_{\text {in }}\right)\right)$;

$$
\int_{\Omega} \varrho \mathbf{u}(\tau, \cdot) \cdot \varphi(\tau, \cdot) \mathrm{d} x-\int_{\Omega} \varrho_{0} \mathbf{u}_{0}(\cdot) \varphi(0, \cdot) \mathrm{d} x
$$




$$
=\int_{0}^{\tau} \int_{\Omega}\left(\varrho \mathbf{u} \partial_{t} \boldsymbol{\varphi}+\varrho \mathbf{u} \otimes \mathbf{u}: \nabla_{x} \boldsymbol{\varphi}+\overline{p(\varrho)} \operatorname{div}_{x} \boldsymbol{\varphi}\right) \mathrm{d} x \mathrm{~d} t-\int_{0}^{\tau} \int_{\Omega} \mathbb{S}\left(\nabla_{x} \mathbf{u}\right): \nabla_{x} \boldsymbol{\varphi} \mathrm{d} x \mathrm{~d} t
$$

for any $\tau \in[0, T]$ and any $\varphi \in C_{c}^{1}\left([0, T] \times \Omega ; R^{3}\right)$. We can perform the weak limit in the renormalized continuity equation (5.3) for $\left(\varrho_{\delta}, \mathbf{u}_{\delta}\right)$. We obtain, by the same token,

$$
\begin{gathered}
\int_{\Omega}(\overline{b(\varrho)} \mathbf{u})(\tau) \varphi(\tau) \mathrm{d} x-\int_{\Omega} b\left(\varrho_{0}\right) \mathbf{u}_{0} \varphi(0) \mathrm{d} x= \\
\int_{0}^{\tau} \int_{\Omega}\left(\overline{b(\varrho)} \mathbf{u} \cdot \nabla_{x} \varphi-\varphi \overline{\left(b^{\prime}(\varrho) \varrho-b(\varrho)\right) \operatorname{div}_{x} \mathbf{u}}\right) \mathrm{d} x \mathrm{~d} t-\int_{0}^{\tau} \int_{\Gamma_{\mathrm{in}}} b\left(\varrho_{B}\right) \mathbf{u}_{B} \cdot \mathbf{n} \varphi \mathrm{d} S_{x} \mathrm{~d} t
\end{gathered}
$$

for any $\varphi \in C^{1}\left([0, T] \times\left(\Omega \cup \Gamma_{\text {in }}\right)\right)$, with any $b$ satisfying conditions $(2.14)_{p=\gamma}$. (Here again $\overline{b(\varrho, \mathbf{u})}$ denotes weak limit of the sequence $b\left(\varrho_{\delta}, \mathbf{u}_{\delta}\right)$ (in $\left.L^{1}\left(Q_{T}\right).\right)$ )

It remains to show that

$$
\overline{p(\varrho)}=p(\varrho) .
$$

The rest of this section is devoted to the proof of this identity. This is equivalent to show that $\varrho_{\delta} \rightarrow \varrho$ a.e. in $Q_{T}$.

\subsection{Effective viscous flux identity}

We now perform similar reasoning as in Section 5.3. Since however functions $\varrho$ and $\varrho \log \varrho$ do not possess enough summability, we shall replace them by convenient truncations $T_{k}(\varrho)$ and $L_{k}(\varrho)$, where $T_{k}(\varrho)$ is defined in (3.11) and

$$
L_{k}(\varrho)=\varrho \int_{1}^{\varrho} \frac{T_{k}(z)}{z} \mathrm{~d} z
$$

We shall repeat the process described in Section 5.3 with $T_{k}\left(\varrho_{\delta}\right)$ resp. $\overline{T_{k}(\varrho)}$ instead of $\varrho_{\delta}, \varrho$ : Following [11], we shall use in the approximating momentum equation $(5.4)$ (where $\left.(\varrho, \mathbf{u})=\left(\varrho_{\delta}, \mathbf{u}_{\delta}\right)\right)$ test function

$$
\varphi(t, x)=\psi(t) \phi(x) \nabla_{x} \Delta^{-1}\left(T_{k}\left(\varrho_{\delta}\right) \phi\right), \quad \psi \in C_{c}^{1}(0, T), \phi \in C_{c}^{1}(\Omega)
$$

and in the limiting momentum equation (6.8) test function

$$
\varphi(t, x)=\psi(t) \phi(x) \nabla_{x} \Delta^{-1}\left(\overline{T_{k}(\varrho)} \phi\right), \quad \psi \in C_{c}^{1}(0, T), \phi \in C_{c}^{1}(\Omega)
$$

subtract both identities and perform the limit $\delta \rightarrow 0$. This leads to equation

$$
\begin{aligned}
& \int_{0}^{T} \int_{\Omega} \psi \phi^{2}\left(\overline{p(\varrho) T_{k}(\varrho)}-(2 \mu+\lambda) \overline{T_{k}(\varrho)} \operatorname{divu}\right) \mathrm{d} x \mathrm{~d} t-\int_{0}^{T} \int_{\Omega} \psi \phi^{2}\left(\overline{p_{\delta}(\varrho) T_{k}(\varrho)}-(2 \mu+\lambda) \overline{T_{k}(\varrho) \operatorname{divu}}\right) \mathrm{d} x \mathrm{~d} t \\
& =\int_{0}^{T} \int_{\Omega} \psi \phi \mathbf{u} \cdot\left(\overline{T_{k}(\varrho)} \mathscr{R} \cdot(\varrho \mathbf{u} \phi)-\varrho \mathbf{u} \cdot \mathscr{R}\left(\overline{T_{k}(\varrho)} \phi\right)\right) \mathrm{d} x \mathrm{~d} t
\end{aligned}
$$




$$
-\lim _{\delta \rightarrow 0} \int_{0}^{T} \int_{\Omega} \psi \phi \mathbf{u}_{\delta} \cdot\left(T_{k}\left(\varrho_{\delta}\right) \mathscr{R} \cdot\left(\varrho_{\delta} \mathbf{u}_{\delta} \phi\right)-\varrho_{\delta} \mathbf{u}_{\delta} \cdot \mathscr{R}\left(T_{k}\left(\varrho_{\delta}\right) \phi\right)\right) \mathrm{d} x \mathrm{~d} t .
$$

Renormalized continuity equation $(5.3)_{\left(\varrho_{\delta}, \mathbf{u}_{\delta}\right)}$ and its weak limit (6.9) with $b=T_{k}$ play in this calculation an important role. Due to the compact support of $\phi$, the non homogeneity of the boundary data is irrelevant. The right hand side of the last identity is zero by div-curl lemma. Consequently, we get the effective viscous flux identity

$$
\overline{p(\varrho) T_{k}(\varrho)}-\overline{p(\varrho)} \overline{T_{k}(\varrho)}=(2 \mu+\lambda)\left(\overline{T_{k}(\varrho) \operatorname{div} \mathbf{u}}-\overline{T_{k}(\varrho)} \operatorname{div} \mathbf{u}\right) .
$$

The details of this calculus and reasoning can be found in [11, Lemma 3.2], [7], [17] or [9, Chapter 3]. If $p$ would be non decreasing we would have $(2 \mu+\lambda)\left(\overline{T_{k}(\varrho) \operatorname{div} \mathbf{u}}-\overline{T_{k}(\varrho)} \operatorname{div} \mathbf{u}\right) \geq 0$ (according to e.g. $[9$, Theorem 10.19]) and we could stop this part of argumentation at this place. In the general case, we must continue.

Writing $p=p-\mathfrak{p}$ and recalling that $p$ is non decreasing, we deduce from identity (6.11),

$$
(2 \mu+\lambda)\left(\overline{T_{k}(\varrho)} \operatorname{div} \mathbf{u}-\overline{T_{k}(\varrho) \operatorname{div} \mathbf{u}}\right) \leq \overline{\mathfrak{p}(\varrho) T_{k}(\varrho)}-\overline{\mathfrak{p}(\varrho)} \overline{T_{k}(\varrho)}
$$

Next, we realize (by employing essentially the lower-weak semi-continuity of norms) that

$$
\limsup _{k \rightarrow \infty}\left\|\overline{T_{k}(\varrho)}-\varrho\right\|_{L^{1}\left(Q_{T}\right)}=0, \quad \lim _{k \rightarrow \infty}\left\|\overline{\mathfrak{p}(\varrho) T_{k}(\varrho)}-\overline{\mathfrak{p}(\varrho)} \varrho\right\|_{L^{1}\left(Q_{T}\right)}=0
$$

whence

$$
\limsup _{k \rightarrow \infty} \int_{0}^{\tau} \int_{\Omega}\left(\overline{\mathfrak{p}(\varrho) T_{k}(\varrho)}-\overline{\mathfrak{p}(\varrho)} \overline{T_{k}(\varrho)}\right) \mathrm{d} x \mathrm{~d} t \leq \int_{0}^{\tau} \int_{\Omega}(\overline{\mathfrak{p}(\varrho) \varrho}-\overline{\mathfrak{p}(\varrho)} \varrho) \mathrm{d} x \mathrm{~d} t
$$

Finally, we employ formulas (5.17), (5.18), similarly as when deriving (5.19), in order to get

$$
(2 \mu+\lambda) \int_{0}^{\tau} \int_{\Omega}\left(\overline{T_{k}(\varrho)} \operatorname{div} \mathbf{u}-\overline{T_{k}(\varrho) \operatorname{div} \mathbf{u}}\right) \mathrm{d} x \mathrm{~d} t \leq c \Lambda(1+\bar{r}) \int_{0}^{\tau} \int_{\Omega}(\overline{\varrho \ln \varrho}-\varrho \ln \varrho) \mathrm{d} x \mathrm{~d} t .
$$

\subsection{Oscillations defect measure}

The main achievement of the present section is the following lemma.

Lemma 6.3. Let $\left(\varrho_{\delta}, \mathbf{u}_{\delta}\right)$ be a sequence constructed in Lemma 5.1. Then

$$
\operatorname{Osc}_{\gamma+1}\left[\varrho_{\delta} \rightarrow \varrho\right]\left(Q_{T}\right)<\infty .
$$

The quantity $\operatorname{osc}_{\gamma+1}\left[\varrho_{\delta} \rightarrow \varrho\right]\left(Q_{T}\right)$ is defined in (3.10)

It is well known that Lemma 6.3 follows from the effective viscous flux identity, see [11, Lemma 4.3], [7], [17] for the detailed proof. To see this fact, we observe that there is non-decreasing $p_{m} \in C[0, \infty)$ and bounded $\mathfrak{p}_{b} \in C[0, \infty)$ such that

$$
p(\varrho)=\frac{a}{2 \gamma} \varrho^{\gamma}+p_{m}(\varrho)-\mathfrak{p}_{b}
$$


Indeed, one may take $p_{m}=p-\frac{a}{2 \gamma} \varrho^{\gamma}+b \eta(\varrho) \min \{\underline{r}, \varrho\}, \mathfrak{p}_{b}(\varrho)=\mathfrak{p}(\varrho)-b \eta(\varrho) \min \{\underline{r}, \varrho\}$, where $\underline{r}$ solves equation $a s^{\gamma-1}-2 b=0$ and $\eta \in C_{c}^{1}[0, \infty), \eta(s)=1$ for $s \in[0, R), 0 \leq-\eta^{\prime}(s) \leq \frac{1}{R}$ with $R$ sufficiently large. With this decomposition, effective viscous flux identity (6.11) can be rewritten as follows

$$
\begin{gathered}
\frac{a}{2 \gamma} \int_{0}^{T} \int_{\Omega}\left(\overline{\varrho^{\gamma} T_{k}(\varrho)}-\overline{\varrho^{\gamma}} \overline{T_{k}(\varrho)}\right) \mathrm{d} x \mathrm{~d} t+\int_{0}^{T} \int_{\Omega}\left(\overline{p_{m}(\varrho) T_{k}(\varrho)}-\overline{p_{m}(\varrho)} \overline{T_{k}(\varrho)}\right) \mathrm{d} x \mathrm{~d} t \\
=\limsup _{\delta \rightarrow 0} \sum_{i=1}^{3} I_{\delta}^{i},
\end{gathered}
$$

where

$$
\begin{gathered}
I_{\delta}^{1}=(2 \mu+\lambda) \int_{0}^{T} \int_{\Omega}\left(T_{k}(\varrho \delta)-T_{k}(\varrho)\right) \operatorname{div}_{x} \mathbf{u}_{\delta} \mathrm{d} x \mathrm{~d} t \\
I_{\delta}^{2}=(2 \mu+\lambda) \int_{0}^{T} \int_{\Omega}\left(T_{k}(\varrho)-\overline{T_{k}(\varrho)}\right) \operatorname{div}_{x} \mathbf{u}_{\delta} \mathrm{d} x \mathrm{~d} t \\
I_{\delta}^{3}=\int_{0}^{T} \int_{\Omega}\left(\overline{\mathfrak{p}_{b}(\varrho) T_{k}(\varrho)}-\overline{\mathfrak{p}_{b}(\varrho)} \overline{T_{k}(\varrho)}\right) \mathrm{d} x \mathrm{~d} t
\end{gathered}
$$

We first observe that the second integral at the left hand side is non negative (indeed, $p_{m}$ is nondecreasing and we can use Theorem 10.19 in [9]). Second, we employ the Hölder inequality and interpolation together with the lower weak semi-continuity of norms and bounds $(6.2-6.3)$ to estimate integrals $I_{\delta}^{1}, I_{\delta}^{2}$ in order to get

$$
\left|I_{\delta}^{1}+I_{\delta}^{2}\right| \leq c\left[\operatorname{osc}_{\gamma+1}\left[\varrho_{\delta} \rightarrow \varrho\right]\left(Q_{T}\right)\right]^{\frac{1}{2 \gamma}}
$$

with $c>0$ independent of $\mathrm{k}$. Finally, since $\mathfrak{p}_{b}$ is continuous with compact support, integral $\left|I_{\delta}^{3}\right|$ is bounded by an universal constant $c=c\left(\mathfrak{p}_{b}\right)>0$.

Next we write, as in [11]

$$
\begin{gathered}
\int_{0}^{T} \int_{\Omega}\left(\overline{\varrho^{\gamma} T_{k}(\varrho)}-\overline{\varrho^{\gamma}} \overline{T_{k}(\varrho)}\right) \mathrm{d} x \mathrm{~d} t= \\
\limsup _{\delta \rightarrow 0} \int_{0}^{T} \int_{\Omega}\left(\varrho_{\delta}^{\gamma}-\varrho^{\gamma}\right)\left(T_{k}\left(\varrho_{\delta}\right)-T_{k}(\varrho)\right) \mathrm{d} x \mathrm{~d} t+\int_{0}^{T} \int_{\Omega}\left(\varrho^{\gamma}-\overline{\varrho^{\gamma}}\right)\left(\overline{T_{k}(\varrho)}-T_{k}(\varrho)\right) \mathrm{d} x \mathrm{~d} t \\
\geq \limsup _{\delta \rightarrow 0} \int_{0}^{T} \int_{\Omega}\left|T_{k}\left(\varrho_{\delta}\right)-T_{k}(\varrho)\right|^{\gamma+1} \mathrm{~d} x \mathrm{~d} t,
\end{gathered}
$$

where we have employed convexity of $\varrho \mapsto \varrho^{\gamma}$ and concavity of $\varrho \mapsto T_{k}(\varrho)$ on $[0, \infty)$, and algebraic inequality

$$
|a-b|^{\gamma} \leq\left|a^{\gamma}-b^{\gamma}\right| \text { and }|a-b| \geq\left|T_{k}(a)-T_{k}(b)\right|, \quad(a, b) \in[0, \infty)^{2} .
$$

Inserting the last inequality into (6.16) yields (in combination with estimates of integrals $I_{\delta}^{1}-I_{\delta}^{3}$ ) the statement of Lemma 6.3. 


\subsection{Strong convergence of density}

Since couple $(\varrho, \mathbf{u})$ verifies continuity equation $(2.6)$, it verifies also renormalized continuity equation (2.13) in view of Lemma 3.2. In view of Remark 2.5 we can take in the latter equation $b=L_{k}$. We get, in particular,

$$
\begin{gathered}
\int_{\Omega} L_{k}(\varrho(\tau, x)) \varphi(x) \mathrm{d} x-\int_{\Omega} L_{k}\left(\varrho_{0}\right) \varphi(x) \mathrm{d} x \\
=\int_{0}^{\tau} \int_{\Omega}\left(L_{k}(\varrho) \mathbf{u} \cdot \nabla_{x} \varphi-\varphi T_{k}(\varrho) \operatorname{div}_{x} \mathbf{u}\right) \mathrm{d} x \mathrm{~d} t+\int_{0}^{\tau} \int_{\partial \Omega} L_{k}\left(\varrho_{B}\right) \mathbf{u}_{B} \cdot \mathbf{n} \varphi \mathrm{d} S_{x} \mathrm{~d} t,
\end{gathered}
$$

with any $\varphi \in C_{c}^{1}\left(\Omega \cup \Gamma_{\text {in }}\right)$ and $\tau \in[0, T]$. On the other hand, equation (6.9) with $b=L_{k}$ reads,

$$
\begin{gathered}
\int_{\Omega} \overline{L_{k}(\varrho)}(\tau, x) \varphi(x) \mathrm{d} x-\int_{\Omega} L_{k}\left(\varrho_{0}\right) \varphi(x) \mathrm{d} x \\
=\int_{0}^{\tau} \int_{\Omega}\left(\overline{L_{k}(\varrho)} \mathbf{u} \cdot \nabla_{x} \varphi-\varphi \overline{T_{k}(\varrho) \operatorname{div}_{x} \mathbf{u}}\right) \mathrm{d} x \mathrm{~d} t+\int_{0}^{\tau} \int_{\partial \Omega} L_{k}\left(\varrho_{B}\right) \mathbf{u}_{B} \cdot \mathbf{n} \varphi \mathrm{d} S_{x} \mathrm{~d} t,
\end{gathered}
$$

where $\varphi \in C_{c}^{1}\left(\Omega \cup \Gamma_{\text {in }}\right)$ and $\tau \in[0, T]$. Subtracting (6.19) and (6.18) yields

$$
\begin{gathered}
\int_{\Omega}\left(\overline{L_{k}(\varrho)}-L_{k}(\varrho)\right)(\tau, x) \varphi(x) \mathrm{d} x-\int_{0}^{\tau} \int_{\Omega}\left(\overline{L_{k}(\varrho)}-L_{k}(\varrho)\right)\left(\mathbf{u}-\tilde{\mathbf{u}}_{B}\right) \cdot \nabla_{x} \varphi \mathrm{d} x \mathrm{~d} t \\
-\int_{0}^{\tau} \int_{\Omega}\left(\overline{L_{k}(\varrho)}-L_{k}(\varrho)\right) \tilde{\mathbf{u}}_{B} \cdot \nabla_{x} \varphi=\int_{0}^{\tau} \int_{\Omega} \varphi\left(T_{k}(\varrho) \operatorname{div}_{x} \mathbf{u}-\overline{T_{k}(\varrho) \operatorname{div}_{x} \mathbf{u}}\right) \mathrm{d} x \mathrm{~d} t \mathrm{~d} x \mathrm{~d} t
\end{gathered}
$$

with any $\varphi \in C_{c}^{1}\left(\Omega \cup \Gamma_{\text {in }}\right)$ and $\tau \in[0, T]$, where $\tilde{\mathbf{u}}_{B}$ is defined in Lemma 2.2 .

Now we consider the family of test functions $\varphi_{\delta}$ defined in (5.27). By the same reasoning as in (5.26-5.29) we deduce

$$
\begin{gathered}
\int_{0}^{T} \int_{\Omega}\left[L_{k}(\varrho)-\overline{L_{k}(\varrho)}\right]\left(\mathbf{u}-\tilde{\mathbf{u}}_{B}\right) \cdot \nabla_{x} \varphi_{\delta} \mathrm{d} x \mathrm{~d} t \rightarrow 0 \text { as } \delta \rightarrow 0, \\
\liminf _{\delta \rightarrow 0} \int_{0}^{T} \int_{\Omega}\left[L_{k}(\varrho)-\overline{L_{k}(\varrho)}\right] \tilde{\mathbf{u}}_{B} \cdot \nabla_{x} \varphi_{\delta} \mathrm{d} x \mathrm{~d} t \geq 0 .
\end{gathered}
$$

Finally we write

$$
\begin{gathered}
\int_{0}^{\tau} \int_{\Omega} \varphi_{\delta}\left(T_{k}(\varrho) \operatorname{div}_{x} \mathbf{u}-\overline{T_{k}(\varrho) \operatorname{div}_{x} \mathbf{u}}\right) \mathrm{d} x \mathrm{~d} t \\
=\int_{0}^{T} \int_{\Omega} \varphi_{\delta}\left(T_{k}(\varrho)-\overline{T_{k}(\varrho)}\right) \operatorname{div}_{x} \mathbf{u} \mathrm{d} x \mathrm{~d} t+\int_{0}^{\tau} \int_{\Omega} \varphi_{\delta}\left(\overline{T_{k}(\varrho)} \operatorname{div}_{x} \mathbf{u}-\overline{T_{k}(\varrho) \operatorname{div}_{x} \mathbf{u}}\right) \mathrm{d} x \mathrm{~d} t,
\end{gathered}
$$

where the first term converges to 0 as $k \rightarrow \infty$ by virtue of (6.14) and interpolation estimate (indeed, $\left\|T_{k}(\varrho)-\overline{T_{k}(\varrho)}\right\|_{L^{1}\left(Q_{T}\right)} \rightarrow 0$ as $k \rightarrow \infty$ by virtue of definition of $T_{k}$ and lower weak semi-continuity of norms), while the second term is bounded from above by the expression at the right hand side of (6.13). 
Coming with all this information back to (6.20) with $\varphi=\varphi_{\delta}$, and performing first limit $\delta \rightarrow 0$ and then limit $k \rightarrow \infty$ we conclude that

$$
\int_{\Omega}(\overline{\varrho \log \varrho}-\varrho \log \varrho)(\tau, x) \mathrm{d} x \leq c \Lambda(1+\bar{r}) \int_{0}^{\tau} \int_{\Omega}(\overline{\varrho \log \varrho}-\varrho \log \varrho) \mathrm{d} x \mathrm{~d} t
$$

for all $\varphi \in C_{c}^{1}\left(\Omega \cup \Gamma_{\text {in }}\right), \varphi \geq 0, \tau \in[0, T]$. Consequently, by the Gronwall lemma

$$
\int_{\Omega}(\overline{\varrho \log \varrho}-\varrho \log \varrho)(\tau, x) \mathrm{d} x \leq 0 .
$$

This means a.e. in $Q_{T}$ convergence of $\varrho_{\delta}$ to $\varrho$ and consequently the identity $\overline{p(\varrho)}=p(\varrho)$.

The passage $\delta \rightarrow 0$ from the energy inequality (5.5) (with $\left(\varrho_{\delta}, \mathbf{u}_{\delta}\right)$ ) to the final energy inequality (2.9) will be done in the same way as in Section 5.5.

We have so far performed the whole proof with initial data satisfying (4.13). We notice that this is without loss of generality. Indeed finite energy initial data (2.15) can be easily approximated on the level $\delta$ by initial data (4.13) in the way suggested in [11, Section 4]. This concludes the proof of Theorem 2.4 .

\section{References}

[1] D. Bresch and P.-E. Jabin. Global existence of weak solutions for compresssible Navier-Stokes equations: Thermodynamically unstable pressure and anisotropic viscous stress tensor. 2015. arxiv preprint No. 1507.04629v1.

[2] S. Benzoni-Gavage. Calcul différentiel et équations différentielles. Dunod. 2010.

[3] J. Bergh, Löfsröm. Interpolation spaces. An Introduction Springer. Berlin-Heidelberk-New York 1976

[4] R. Denk, M. Hieber, and J. Prüss. Optimal $L p-L q$-estimates for parabolic boundary value problems with inhomogeneous data Math. Z.257:193224, 2007

[5] R. Denk, M. Hieber, and J. Prüss. R-boundedness, Fourier multipliers and problems of elliptic and parabolic type. Mem. Am. Math. Soc., 788, 2003.

[6] R.J. DiPerna and P.-L. Lions. Ordinary differential equations, transport theory and Sobolev spaces. Invent. Math., 98:511-547, 1989.

[7] E. Feireisl. Dynamics of viscous compressible fluids. Oxford University Press, Oxford, 2004.

[8] E. Feireisl Compressible Navier-Stokes equations with a non-monotone pressure law. J. Differential Equations 184: 97-108, 2002. 
[9] E. Feireisl and A. Novotný. Singular limits in thermodynamics of viscous fluids. Birkhauser, Basel, 2009.

[10] E. Feireisl and A. Novotný. Stationary solutions to the compressible Navier-Stokes system with general boundary conditions Preprint Nečas Center for Mathematical Modeling, Charles University, Prague, 2017

[11] E. Feireisl, A. Novotný, and H. Petzeltová. On the existence of globally defined weak solutions to the Navier-Stokes equations of compressible isentropic fluids. J. Math. Fluid Mech., 3:358-392, 2001.

[12] R.L. Foote. Regularity of the distance function. Proc. Amer. Math. Soc., 92:153-155, 1984.

[13] V. Girinon. Navier-Stokes equations with nonhomogeneous boundary conditions in a bounded three-dimensional domain. J. Math. Fluid Mech. 13: 309339, 2011

[14] P.-L. Lions. Mathematical topics in fluid dynamics, Vol.2, Compressible models. Oxford Science Publication, Oxford, 1998.

[15] P. B. Mucha and T. Piasecki. Compressible perturbation of Poiseuille type flow. J. Math. Pures Appl. (9), 102(2):338-363, 2014.

[16] S. Novo. Compressible Navier-Stokes model with inflow-outflow boundary conditions. J. Math. Fluid Mech., 7(4):485-514, 2005.

[17] A. Novotný and I. Straškraba. Introduction to the mathematical theory of compressible flow. Oxford University Press, Oxford, 2004.

[18] T. Piasecki. On an inhomogeneous slip-inflow boundary value problem for a steady flow of a viscous compressible fluid in a cylindrical domain. J. Differential Equations, 248(8):2171-2198, 2010.

[19] T. Piasecki and M. Pokorný. Strong solutions to the Navier-Stokes-Fourier system with slip-inflow boundary conditions. ZAMM Z. Angew. Math. Mech., 94(12):1035-1057, 2014.

[20] P. I. Plotnikov, E. V. Ruban, and J. Sokolowski. Inhomogeneous boundary value problems for compressible Navier-Stokes equations: well-posedness and sensitivity analysis. SIAM J. Math. Anal., 40(3):1152-1200, 2008.

[21] P. I. Plotnikov, E. V. Ruban, and J. Sokolowski. Inhomogeneous boundary value problems for compressible Navier-Stokes and transport equations. J. Math. Pures Appl. (9), 92(2):113-162, 2009 .

[22] M.E. Taylor. Partial Differential Equations (Basic theory). Springer, 2011

[23] A. Valli and M. Zajaczkowski. Navier-Stokes equations for compressible fluids: Global existence and qualitative properties of the solutions in the general case. Commun. Math. Phys., 103:259-296, 1986. 